\title{
Fast Demand Control in Smart Grid Communications with User-in-the-Loop (UIL) Method
}

\author{
by \\ Inan Korkmaz \\ A thesis submitted to the \\ Faculty of Graduate and Postdoctoral Affairs \\ in partial fulfillment of the requirements for the degree of
}

Master of Applied Science in Electrical and Computer Engineering

Ottawa-Carleton Institute for Electrical and Computer Engineering

Department of Systems and Computer Engineering

Carleton University

Ottawa, Ontario

May, 2015

(C) Copyright

Inan Korkmaz, 2015 
The undersigned hereby recommends to the

Faculty of Graduate and Postdoctoral Affairs acceptance of the thesis

\title{
Fast Demand Control in Smart Grid Communications with User-in-the-Loop (UIL) Method
}

\author{
submitted by Inan Korkmaz \\ in partial fulfillment of the requirements for the degree of \\ Master of Applied Science in Electrical and Computer Engineering
}

Professor Halim Yanikomeroglu, Thesis Supervisor

Professor Roshdy Hafez, Chair, Department of Systems and Computer Engineering

Ottawa-Carleton Institute for Electrical and Computer Engineering

Department of Systems and Computer Engineering

Carleton University

May, 2015 


\section{Abstract}

Recently, household energy consumption has been highly increasing, although there is a decrease in the source of energy. Consequently, the base price of electricity has been rising. Thus, there are a variety of approaches to save electricity costs.

One proposed approach is the User-in-the Loop (UIL) method, in which the consumers (i.e. users) are given satisfactory incentives (for instance decreasing the base price of the electricity) to postpone their demand until low peak hours. To explore the effectiveness of this method, this study enrolls the open-loop control model and the closed-loop control model. While the open-loop control model is related to consumers' decisions of the process time relying on the low electricity cost, the closed-loop control model refers to consumer's reactions to the system's output and given incentives by electricity suppliers. To investigate the effectiveness of UIL, both control models are examined under three types of pricing models; namely, the fixed, current, and the dynamic pricing models. Whereas the base price of the electricity is steady in the fixed pricing model, under the current pricing model, it varies according to specified time periods in the current pricing model. Additionally, we propose the dynamic pricing model, in which the base price of the electricity is updated every 5 minutes based on a feedback message received from users. This feedback message is transmitted through ZegBee communication collecting the total amount of the electricity consumption of all home appliances.

As a result of this study, we found that there is an inverse relationship between the number of users and the efficiency of UIL with the dynamic pricing model. 


\section{Acknowledgments}

First and foremost, I am most grateful to Allah (God), the Creator and Sustainer of the Universe, for giving me the ability to complete this work. I wish to extend my special thanks to my supervisor, Dr. Halim Yanikomeroglu, for his support and guidance towards achieving the completion of this thesis. I would like to thank Dr. Rainer Schoenen, Postdoctoral fellow in our group, for his invaluable help and suggestions throughout my research. Thanks to Ibrahim Aydin, for the feedback towards improving my work. Thanks to the departmental staff for their administrative and technical support. I would like to thank all of my friends with whom I studied; we encouraged one another throughout the course of our studies. I am deeply grateful to my parents for raising me and for teaching me the most important things in life. A huge thanks to my wife for her moral support and for always keeping me cheered up. 


\section{List of Abbreviations}

5G: $5^{\text {th }}$ Generation

AC: Air Conditioner

ac: Alternative Current

AMI: Advanced Metering Infrastructure

AMR: Automatic Metering Reading

BW: Bandwidth

CDF: Cumulative Distribution Function

CLC: Closed-Loop Control

EU: End User

EUP: End User Price

FDC: Fast Demand Control

IESO: Independent Electricity System Operator

IoE: Internet of Everything

IoTs: Internet of Things

IPv4: Internet Protocol version 4

IPv6: Internet Protocol version 6

M2M: Machine to Machine Communication

NANs: Neighborhood Area Networks

OEB: Ontario Energy Board

OFDM: Orthogonal Frequency Division Multiplexing

OLC: Open-Loop Control

PDF: Power Spectral Density

PID: Proportion Integral Differential

PLC: Power Line Communication

RF: Radio Frequency

RPP: Regulated Price Plan

SG: Smart Grid

SM: Smart Meter

UIL: User-in-the-Loop

UIOL: User-in-the-Open-Loop 


\section{Table of Contents}

Abstract $\quad$ iii

Acknowledgments $\quad$ iv

Table of Contents vi vi v

List of Tables $\quad$ ix

List of Figures $\quad$ x

1 Introduction $\quad \mathbf{1}$

$1.15 \mathrm{G}$ definition and Internet of Things (IoTs) . . . . . . . . . . 1

1.2 Fast Demand Control (FDC) in Energy Grid . . . . . . . . . . . . . 4

1.3 Smart Grid Communications Aspects . . . . . . . . . . . . 5

1.3.1 Smart Meter Structure . . . . . . . . . . . . 8

1.3.2 Power Line Communications (PLCs) . . . . . . . . . . . . 14

1.4 UIL Definition and its Properties . . . . . . . . . . . . . . 16

1.5 ZigBee Communications . . . . . . . . . . . . . . . 17

1.5.1 802.15.4 ZigBee Physical Layer . . . . . . . . . . . . . . . . 17

1.5.2 The ZigBee Protocol . . . . . . . . . . . . . . . . 18

1.5.3 Why Do We Need ZigBee Communications? . . . . . . . . . . 18

1.5.4 Mesh Networks . . . . . . . . . . . . . . . . 19

1.5.5 The Concept of Open-Loop Control (OLC) and Closed-Loop Control (CLC) . . . . . . . . . . . . . . . . 22

1.5.6 Problem Statement . . . . . . . . . . . . . . . . 26

1.5.7 Contributions . . . . . . . . . . . . . . . . . 28

1.5.8 Thesis Organization ................ 28 
2 System Definition $\quad 29$

2.1 Pricing Systems Comparisons . . . . . . . . . . . . . . . 29

2.1.1 Fixed Pricing Model . . . . . . . . . . . . . . . . . . 29

2.1.2 Current Pricing Model (Partly Dynamic) . . . . . . . . . . . . 30

2.1.3 Dynamic Pricing Model . . . . . . . . . . . . . . . . . 31

2.2 Simulation Tools . . . . . . . . . . . . . . . . . . 35

2.3 System Model . . . . . . . . . . . . . . . . . . . . 37

2.4 Independent Electricity System Operator (IESO) . . . . . . . . . 45

2.5 Summary of This Chapter . . . . . . . . . . . . . . . . . 47

3 Open-Loop Control Model 48

3.1 Fixed Pricing Model . . . . . . . . . . . . . . . . . 50

3.1.1 One-User One-Appliance Model . . . . . . . . . . . . . . . . . 50

3.1 .2 One-User Multi-Appliance Model . . . . . . . . . . . . . . 51

3.1.3 Multi-User Multi-Appliance Model . . . . . . . . . . . . . . . 52

3.2 Current Pricing Model . . . . . . . . . . . . . . . . . . . 53

3.3 Dynamic Pricing Model . . . . . . . . . . . . . . . . 61

3.3.1 One-User One-Appliance Model . . . . . . . . . . . . . . . . . 61

3.3.2 One-User Multi-Appliance Model . . . . . . . . . . . . . . . . 62

3.3.3 Multi-User Multi-Appliance Model . . . . . . . . . . . . 63

3.4 Summary of This Chapter . . . . . . . . . . . . . . 63

4 Closed-Loop Control Model $\quad 65$

4.1 Fixed Pricing Model . . . . . . . . . . . . . . . . . . . . 70

4.2 Current Model . . . . . . . . . . . . . . . . . . 71

4.3 Dynamic Pricing Model . . . . . . . . . . . . . . . . . . . . 72

4.4 Summary of This Chapter . . . . . . . . . . . . . . . . . . 72

5 Conclusion and Future Works $\quad 74$

5.1 Open-Loop Results . . . . . . . . . . . . . . . . . . . . 74

5.2 Closed-Loop Results . . . . . . . . . . . . . . . 76

5.3 Conclusion . . . . . . . . . . . . . . . . . 79

5.3.1 Improvements Over the Current Pricing Model and Future Works 81

List of References $\quad 83$ 
Appendix A Equations 


\section{List of Tables}

2.1 Improvements in Dynamic, Current, and Fixed Pricing Models (Cent) 46

5.1 Model Pricing Comparisons . . . . . . . . . . . . . . . 79 


\section{List of Figures}

1.1 Internet of Things (IoTs) Structure [1] . . . . . . . . . . . 2

1.2 Smart Grid Communication Infrastructures . . . . . . . . . . 7

1.3 A Smart Meter $(\mathrm{SM})$ Structure . . . . . . . . . . . . . 8

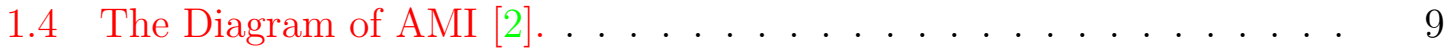

1.5 Time of Use (TOU) Ontario [3] . . . . . . . . . . . . . . . 10

1.6 Tiered Pricing (Ontario) [4] . . . . . . . . . . . . . . . . . . 11

1.7 5-Year Fixed vs. 6-Month RPP [4]. . . . . . . . . . . . . . . . . 12

1.8 Constant vs. Fluctuating Rates [4] . . . . . . . . . . . . . . . 12

1.9 A Typical PLC Model [5]. . . . . . . . . . . . . . . . . 15

1.10 User-in-the-Loop (UIL) Model [6] . . . . . . . . . . . . . . . . . 16

1.11 Mesh Networks . . . . . . . . . . . . . . . . . . . . . . 19

1.12 ZigBee Network $[7] \ldots \ldots \ldots$

1.13 Bluetooth vs. Wi-Fi vs. ZigBee vs. Others $[8] \ldots \ldots \ldots \ldots$

1.14 Open-Loop Model . . . . . . . . . . . . . . . . . . . . . . . . . . 23

1.15 Closed-Loop Control Model . . . . . . . . . . . . . . . . . . . 24

2.1 Demand and Supply . . . . . . . . . . . . . . . . . . 30

2.2 Supply-Demand-Price Relation $[9] \ldots \ldots \ldots$

2.3 On-Off-Peak Hours for Ontario $[10] \ldots \ldots \ldots \ldots$

2.4 Dynamic Pricing for 5 Minutes Cycle . . . . . . . . . . . . . 33

2.5 Green-Yellow-Red Regions for Illinois . . . . . . . . . . . . . . . 34

2.6 Green-Yellow-Red Regions for Ontario . . . . . . . . . . . . . 34

2.7 Sub-Poisson, Poisson, Super Poisson [11] . . . . . . . . . . . . . . 35

2.8 Air Conditioner . . . . . . . . . . . . . . . . . . . . 36

2.9 System Model . . . . . . . . . . . . . . . . . . . . . . . . . . . . . . 37

2.10 Price Added to the System from the Utility Company . . . . . . . . 38

2.11 Backbone of the System Model . . . . . . . . . . . . . . . 38

2.12 Random Selection Among Users . . . . . . . . . . . . . . . . . . 39 
2.13 Main parameter of the System . . . . . . . . . . . . . . . . . 39

2.14 Main Parameter of the Users . . . . . . . . . . . . . . . . . . . 40

2.15 Counting Days with its Parameters . . . . . . . . . . . . . 40

2.16 General View of Appliances . . . . . . . . . . . . . . . . . 41

2.17 Fridge is an Example of non-Shiftable Demand . . . . . . . . . . . 42

2.18 The Recover Parameters for non-Shiftable Demand . . . . . . . . . 42

2.19 Constant Values for Appliance Models . . . . . . . . . . . . . . 43

2.20 Prices are Calculated Based on the Consumed Energy . . . . . . . . . 43

2.21 Calculating the Total Payment . . . . . . . . . . . . . . . . . 44

2.22 Calculating the Total Consumption . . . . . . . . . . . . 44

2.23 Dynamic, Current, and Fixed Pricing Models . . . . . . . . . . . 46

3.1 Example for Open-Loop Control Model . . . . . . . . . . . . . . . 48

3.2 Open-Loop Control Model Road Map . . . . . . . . . . . . . . . . . 49

3.3 Linear Graph . . . . . . . . . . . . . . . . . . . . . . . . . 51

3.4 Fixed Pricing Model . . . . . . . . . . . . . . . . . . . . . . 52

3.5 Current Pricing Rate with Threshold Values . . . . . . . . . . . 54

3.6 PDF, CDF and Histogram of X (Base Price) . . . . . . . . . 55

3.7 One-User One-Appliance Model . . . . . . . . . . . . . . . . . . . . . . 59

3.8 Multi-User Multi-Appliances Model . . . . . . . . . . . . . . . . . 60

3.9 One-User One-Appliance Model . . . . . . . . . . . . . . . . . . . . 62

3.10 Multi-User Multi-Appliance Model . . . . . . . . . . . . . . . . 64

4.1 Closed-Loop Models . . . . . . . . . . . . . . . . . . . . . 66

4.2 PID Controller . . . . . . . . . . . . . . . . . . 67

4.3 Closed-Loop Control Model . . . . . . . . . . . . . . . . . . . 68

4.4 Temporal Probability with Postponed Time [12] . . . . . . . . . . . . 69

4.5 The Outline of Closed-Loop Control Model . . . . . . . . . . . . . 70

4.6 Fixed Pricing System for Closed-Loop Model . . . . . . . . . . . . . . 71

4.7 Current Pricing System for Closed-Loop Model . . . . . . . . . . . 72

4.8 Dynamic Pricing System for Closed-Loop Model . . . . . . . . . . . 73

5.1 Supply Curve . . . . . . . . . . . . . . . . . . . . 75

5.2 Demand Curve . . . . . . . . . . . . . . . . . . 75

5.3 Electricity Price Distribution . . . . . . . . . . . . . 76

5.4 One-User One-Appliance . . . . . . . . . . . . . . . . . . . . 77

5.5 Multi-User Multi-Appliance Model for Open-Loop Control Model . . 78 
5.6 Multi-User Multi-Appliance Model for Closed-Loop Control Model .

5.7 Comparison of Simulated Models with Different Number of Users w/o UIL Method . . . . . . . . . . . . . . . . 80 


\section{Chapter 1}

\section{Introduction}

\subsection{G definition and Internet of Things (IoTs)}

The main idea in a cellular network is to meet the required demand in data rate, coverage, speed, etc. 5G ( $5^{\text {th }}$ generation mobile networks) promises to its users high data rates of several tens of $\mathrm{Mb} / \mathrm{s}$ supported for hundreds of users and $1 \mathrm{~Gb} / \mathrm{s}$ simultaneously to tens of workers on the same office floor. Likewise, $5 \mathrm{G}$ allows up to several 100,000s of simultaneous connections to support massive sensor deployments. Furthermore, it improves coverage and enhances signal efficiency. This technology will be rolled out by 2020 to meet business and consumer demands. Without going into more detail, it is important to mention another aspect of $5 \mathrm{G}$ in regards to its application. 5G should not be considered only for layers 1, 2, or 3. Moreover, 5G comes with its applications which are called Internet of Things (IoT), or Internet of Everything (IoE), as recently introduced in literature, see Figure 1.1. The IoT is the interconnection of uniquely identifiable embedded computing devices within the exist-

ing Internet infrastructure. Typically, IoT is expected to offer advanced connectivity of devices, systems, and services that goes beyond machine-to-machine communications (M2M) and covers a variety of protocols, domains, and applications [13]. The 


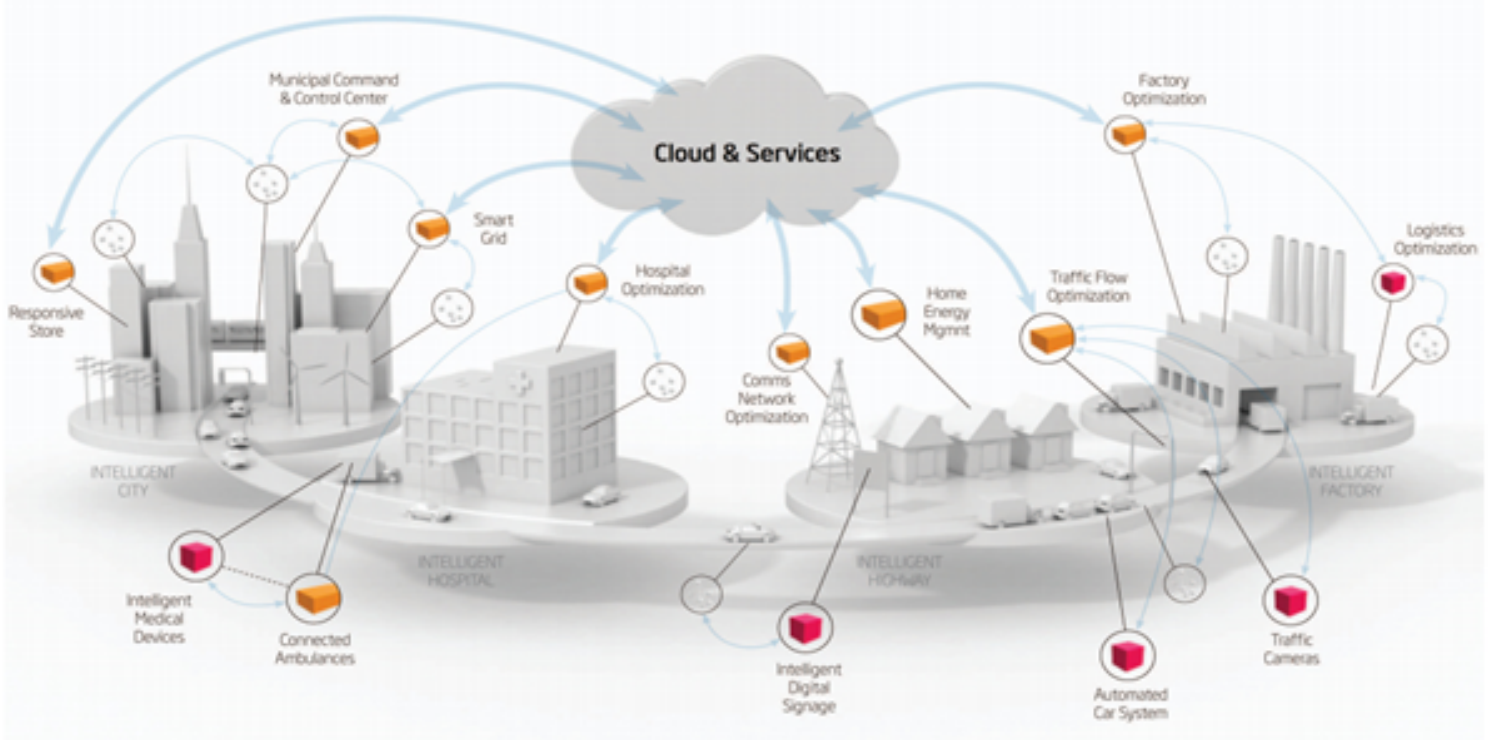

Figure 1.1: Internet of Things (IoTs) Structure [1].

interconnection of these embedded devices (including smart objects), is expected to be used in automation in nearly all fields, while also enabling advanced applications like a smart grid.

The term things as in the 'IoT', can refer to a wide variety of devices such as heart monitoring implants, biochip transponders on farm animals, electric clams in coastal waters, automobiles with built-in sensors, or field operation devices that assist fire-fighters in search and rescue. Current market examples include smart thermostat systems and washer/dryers that utilize Wi-Fi for remote monitoring.

Besides the plethora of new application areas into which Internet connected automation expands, IoT is also expected to generate large amounts of data from diverse locations that is aggregated and very high-velocity, thereby increasing the need to better index, store and process such data. According to Gartner, Inc. (a technology research and advisory corporation), there will be nearly 26 billion devices on the Internet of Things by 2020 [14]. ABI Research estimates that more than 30 
billion devices will be wirelessly connected to the Internet of Things (Internet of Every Things) by 2020 [15]. As per a recent survey and study done by Pew Research Internet Project, a large majority of the technology experts and engaged Internet users who responded $83 \%$ agreed with the notion that the Internet/cloud of things, embedded and wearable computing (and the corresponding dynamic systems) will be widespread and have beneficial effects by 2025. It is clear that the IoT will consist of a large number of devices being connected to the Internet.

Integration with the Internet implies that devices will utilize an IP address as a unique identifier. However, due to the limited address space of IPv4 (which allows for 4.3 billion unique addresses), objects in the IoT will have to use IPv6 to accommodate the significant address space required [16]. Objects in the IoT will not only be devices with sensory capabilities, but they will also provide actuation capabilities (e.g., bulbs or locks controlled over the Internet).

To a large extent, the future of the IoT will not be possible without the support of IPv6; and consequently the global adoption of IPv6 in the coming years will be critical for the successful development of the IoT in the future.

IoTs has ability to network embedded devices with limited CPU, memory, and power resources demonstrates that it can find applications in nearly every field. Such systems could be in charge of collecting information in settings ranging from natural ecosystems to buildings and factories, thereby finding applications in the environmental sensing and urban planning fields.

On the other hand, IoT systems could also be responsible for performing actions, not just sensing things. Intelligent shopping systems, for example, could monitor specific users' purchasing habits in a store by tracking their mobile phones. With the IoT producers can provide users with special offers on their favourite products and locate items of interest which their refrigerator automatically conveys to the 
phone. Additional examples of sensing and actuating are reflected in applications that deal with heat, electricity and energy management, as well as cruise-assisting transportation systems.

\subsection{Fast Demand Control (FDC) in Energy Grid}

After some introduction in the IoT, now it is time to make a definition of Fast Demand Control (FDC). FDC refers to the rapid demand cutbacks that can be achieved "within the flick of a switch", for instance, from turning point of a home appliance, such as an air conditioner, dryer or lights. FDC is the key factor for users (ustomers), for controlling their energy usage instantly called dynamic control of energy usage. Since users are able to use their energy instantly, they are also able to manage the usage time. Before turning on their appliances (dryer, washer, air conditioner, etc.) users are supposed to check the cost of electricity on the screen of smart appliances or cell phones. After seeing the information on the screen, they make a decision based on the lower electricity price range. Users will see the prices instantly from low to high through different indicators (numerical information, coloured indicator, warning beep, a scale, etc.). These indicators give information about the electricity price in each 5 minute cycle. In my model, I use the three universal colour indicators: green colour are used for lower electricity prices. When users see the green colour on the smart device's screen, they understand that it is a suitable time to turn on their appliances. The green colour is chosen based on $\tau_{1}$ value, which is the upper boundary of green colour. Users are supposed to use their appliances or electric cars with the probability of $\left(P_{O N}=1, P_{O F F}=0\right)$ to get incentives. When users turn on their smart appliances or charge electric cars ahead of time, they will get some incentives which can be bonus points to direct discount in electricity bills. Applying 
the model of incentives encourages users to change their demand when the electricity price are low [17]. In the big picture, this kind of regulation ensures the supply and demand equilibrium (balance). Similarly, if the cost of electricity is higher than $\tau_{1}$ but lower than $\tau_{2}$, it is time to indicate a yellow colour for mid-peak range electricity prices. In this region, the users are expected to make a random decision with a probability $\left(P_{O N}=0.5, P_{O F F}=0.5\right)$ that does not impair the household budget. This region is flexible in terms of electricity prices because users see the yellow indicator on their smart phone or appliance. Without thinking of electricity prices, they make a random decision based on the probability of cost versus benefit. Furthermore, higher than yellow bound indicates that users should avoid turning on their appliances during that time. This is when the price is higher than $\tau_{2}$, where the coding is indicated through the colour red. The probability of ON-OFF of appliances will be $\left(P_{O N}=0, P_{O F F}=1\right)$. Without doubt, users have the freedom to turn on their appliances and consume energy during the red range, but it allows them to be aware of the high electricity price during peak hours. By seeing all of the information on the screen, users turn on their appliances [18].

\subsection{Smart Grid Communications Aspects}

The ideas discussed above are components of the smart grid. The smart grid refers to the next generation power grid which upgrades electricity distribution and management by incorporating advanced two-way communication and pervasive capabilities for improved control, efficiency, reliability, and safety. A smart grid delivers electricity between suppliers and consumers using two-way digital technologies. It controls smart appliances in consumer households, reduce costs and increases reliability, efficiency and transparency to save energy. A smart grid is expected to modernize the 
legacy electricity network. It provides automatic monitoring, protecting and optimizing to the operation of the interconnected elements. It covers traditional central generators and/or emerging renewal distributed generators, through a transmission network and distribution system, and to industrial consumer as well as household consumers with their thermostats, electric vehicles, and smart appliances [19]. A smart grid is characterized by the bidirectional connection of electricity and information flows, to create an automated, widely distributed delivery network. It incorporates the legacy electricity grid, with the benefits of modern communication, to deliver realtime information and enable the near-instantaneous balance of supply and demand management. A smart grid is adopted in many technologies that are already in use in industrial applications, such as a wireless network in telecommunications, sensor networks in manufacturing, and now is adopted in new intelligent and interconnected technologies [20]. We can divide the smart grid communications technology into five groups:

- Integrated communications

- Advanced components

- Sensing and measurements

- Improved interfaces and decision support

- Standards and groups.

Figure 1.2 illustrates a general architecture of smart grid communication infrastructures. Smart grids distribute electricity between generators (both traditional power generation and distributed generation sources) and users (industrial, commercial, residential consumers) using bi-directional information flow to control consumers 


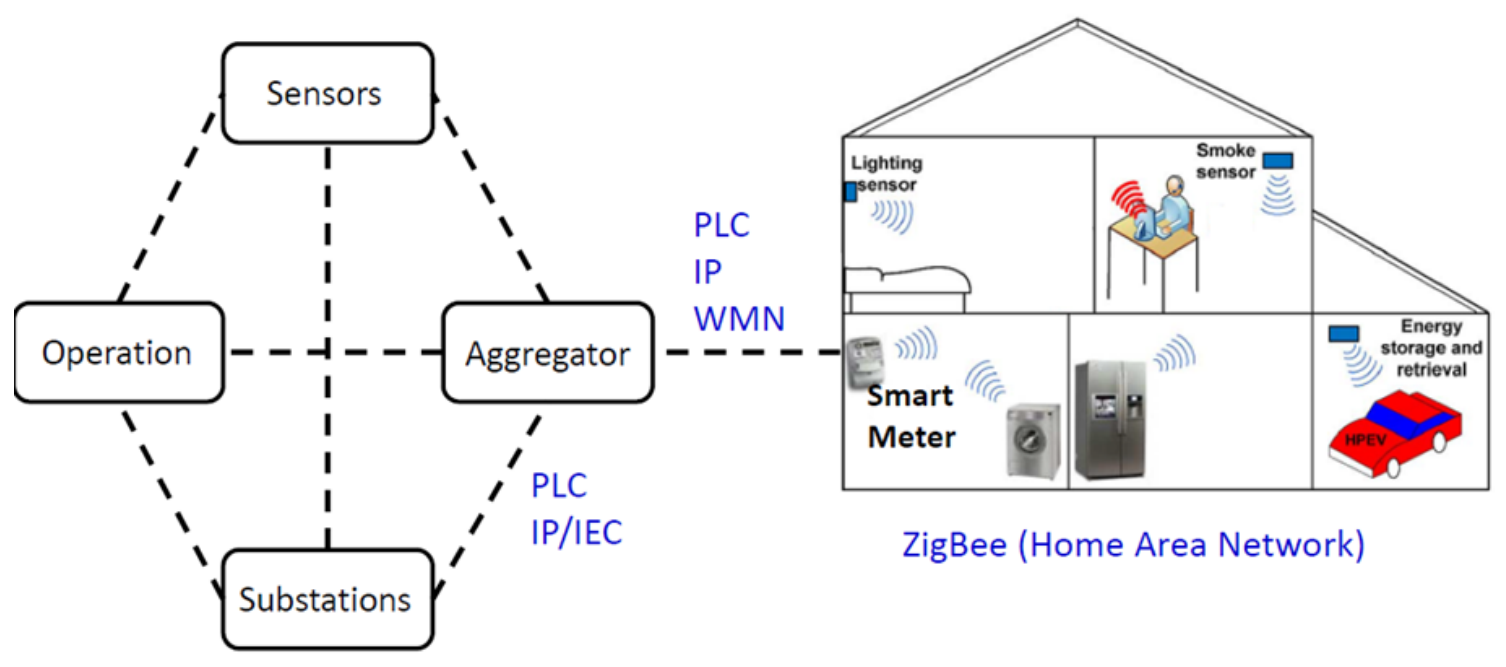

Figure 1.2: Smart Grid Communication Infrastructures

intelligent appliances. Thus, saving energy consumption and reducing the consequent expense, meanwhile increasing system reliability and operation transparency. With a communication infrastructure, the smart metering/monitoring techniques can provide the real-time energy consumption as feedback and correspond to the demand to/from utilities. The network operation centre can retrieve the customer power usage data and the on-line market pricing from data centres, to optimize the electricity generation and distribution, according to the energy consumption [21].

The key point of smart grid communications is the ability of different variables (e.g. intelligent devices, dedicated software, processes, control centre, etc.) to interact through a communication infrastructure [22].

Current electrical utility Wide Area Networks (WANs) consist of hybrid communication, such as wired communications (fiber optics), power line communications (PLC), copperwire line, and various wireless technologies (data communications in cellular networks such as GSM/GPRS/WiMax/WLAN and Cognitive Radio) [23]. 


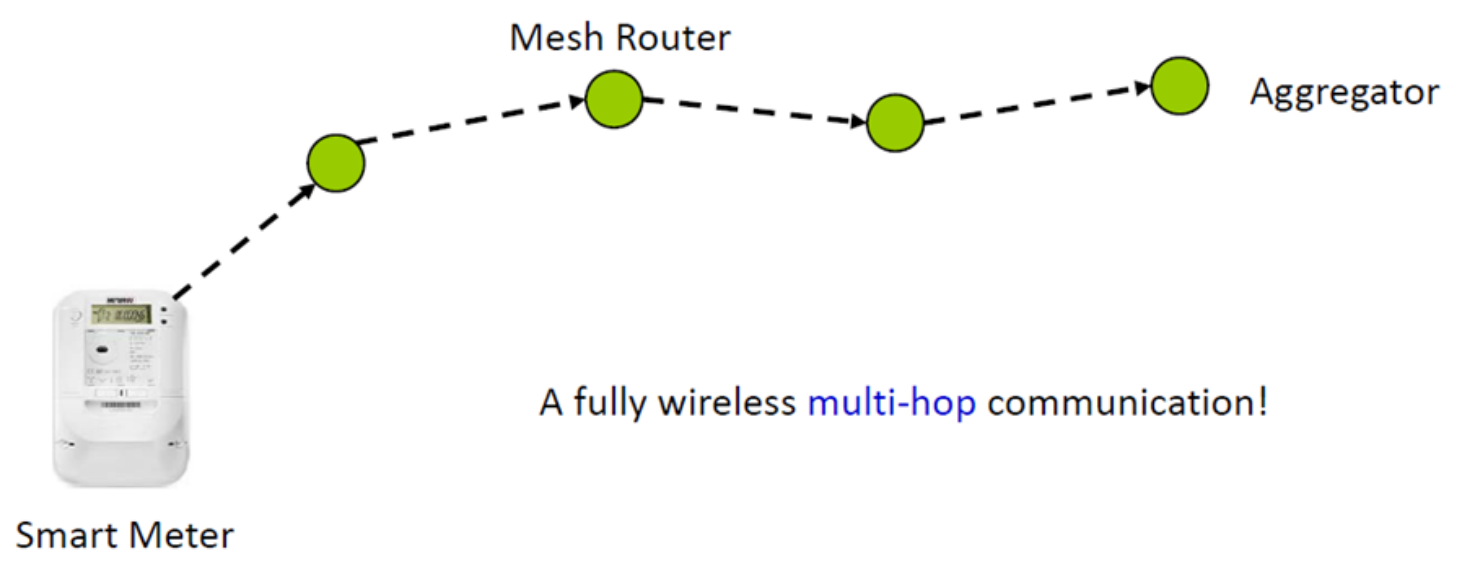

Figure 1.3: A Smart Meter (SM) Structure

\subsubsection{Smart Meter Structure}

The communication is done through wire line or wireless networks by using smart meter technology, see Figure 1.3. Besides, a smart meter monitors and records how much electricity is used in a house. The smart meter measures electricity consumption data over short time periods, usually an hour, and stores this information. Then this data is transmitted to a central computer at the utility site. The data is used to calculate the customers' monthly bills. In our model we propose 5 minute cycles for data transmission between customers and the utility company. This enables users to see their usage instantly, when they decide to operate their appliance.

AMI is a key factor in the smart grid, which is the architecture for automated, twoway communications between the smart meter and the utility company, see Figure 1.4. A smart meter is an advanced meter which identifies power consumption in greater detail than a conventional meter and communicates the collected information back to the utility for load monitoring and billing purposes. Users can be informed of 


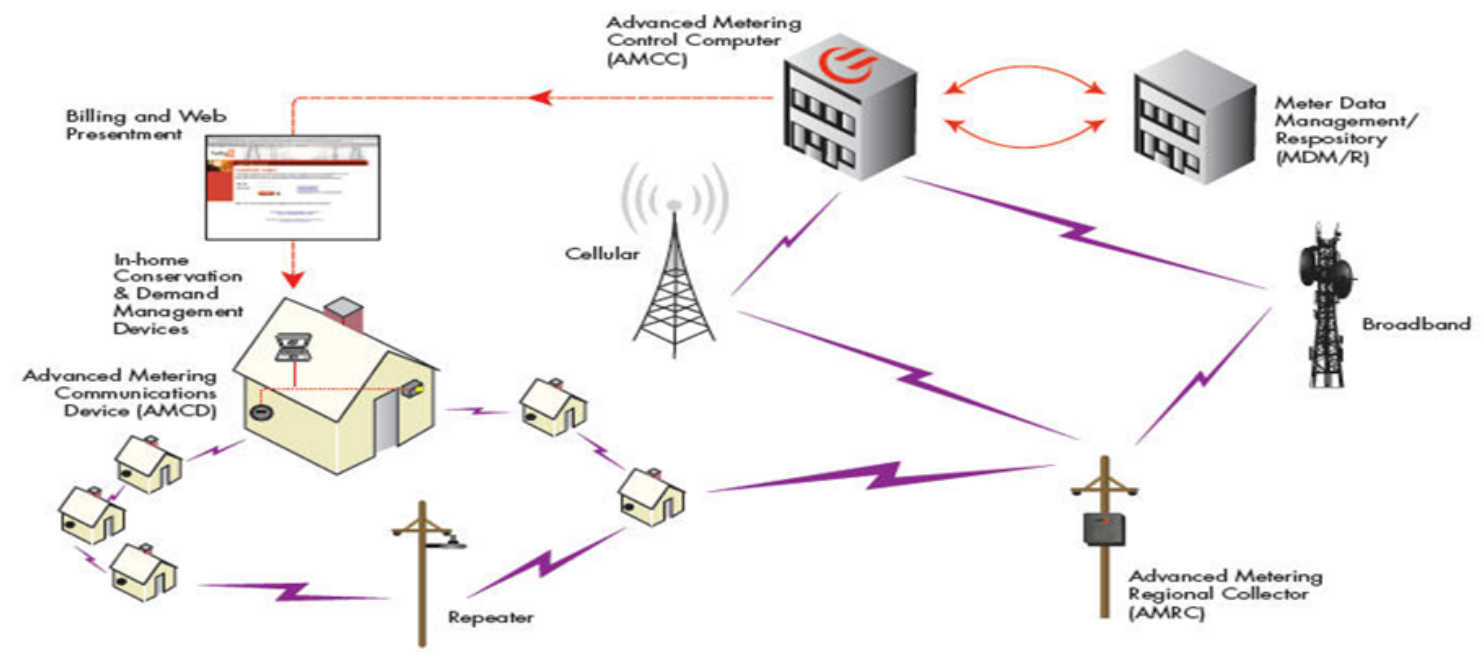

Figure 1.4: The Diagram of AMI [2].

how much power they consume so that they can control the usage and reduce carbon dioxide emission. By managing the peak load through consumers' participation, the utility will likely provide electricity at lower rates for all.

\section{Time-of-Use Pricing}

Smart meters are designed to work with TOU rates, which change during the day; charging more for electricity consumed during peak-demand periods and less for electricity consumed during low-demand periods. By adjusting customers' electricity usage habits slightly, they can take advantage when the cost of electricity is low. When users are transitioned to TOU pricing, their home or business is fitted with a smart meter, which wirelessly transmits their power usage to HydroOne; therefore, the utility company can prepare the electricity bill for customers as accurately as possible. Most importantly, smart meters help users better understand their daily electricity usage and its effects on their monthly bill.

When users know exactly how much electricity they consume and what the price 


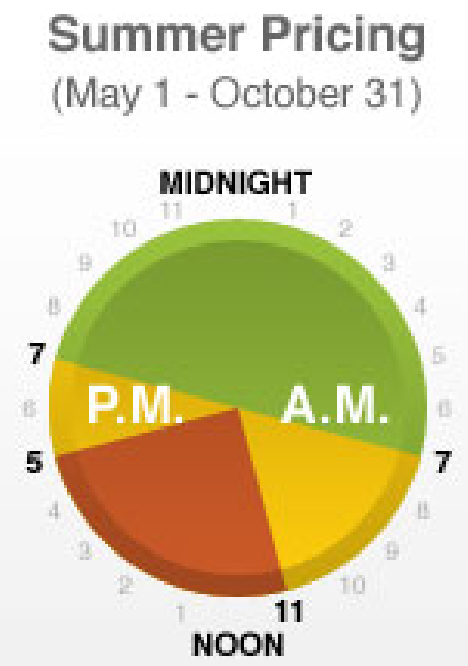

Weekends/Holidays

(All Year)

MIDNIGHT

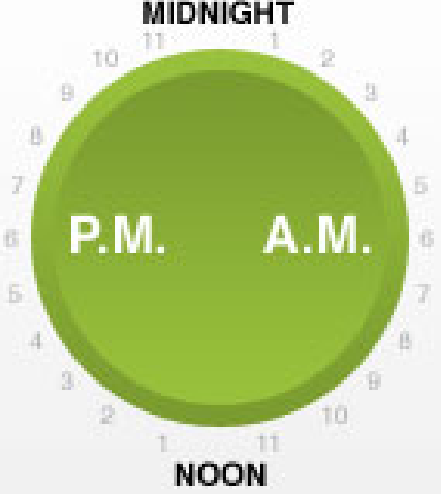

Winter Pricing

(November 1 - April 30)

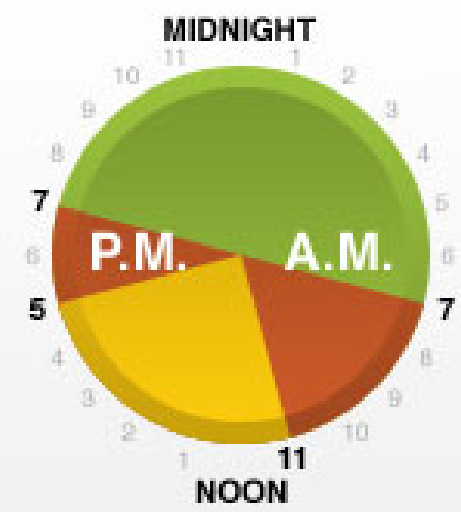

Figure 1.5: Time of Use (TOU) Ontario [3].

will be at a given time, they get the chance to make a smarter decision for energy usage. Users should be able to save money by reducing their electricity usage during the peak hours, through pricing that rewards them for shifting their heaviest electricity usage to off-peak hours, see Figure 1.5.

\section{Tiered (Normal Meter) Pricing}

There are two different prices on monthly bills and slight changes between winter and summer seasons because the customers are on a tiered pricing plan (RPP). Two different prices are set twice a year by the Ontario Energy Board. One price applies up to a certain threshold and a higher price applies if the users go over this threshold, see Figure 1.6. 


\section{Season Period Price-Threshold}

\begin{tabular}{|c|c|c|c|}
\hline$\pi$ & Summer & May - Oct & $\begin{array}{l}\text { Up to } 600 \mathrm{kWh}=\mathbf{8 . 6} \phi / \mathrm{kWh} \\
\text { More than } 600 \mathrm{kWh}=10.1 \mathrm{c} / \mathrm{kWh} \\
\text { Prices effective from May } 1,2014 \text { to } \\
\text { Octo ber } \mathbf{3 1 , 2 0 1 4}\end{array}$ \\
\hline$\frac{3}{4}$ & Winter & $\begin{array}{l}\text { Nov. - } \\
\text { Apr. }\end{array}$ & $\begin{array}{l}\text { Up to } 1,000 \mathrm{kWh}=\mathbf{8 . 8} \mathbf{c / k W h} \\
\text { More than } 1,000 \mathrm{kWh}=10.3 \mathrm{c} / \mathrm{kWh} \\
\text { Prices effective from November } \mathbf{1 , 2 0 1 4} \text { to } \\
\text { April } \mathbf{3 0 , 2 0 1 5}\end{array}$ \\
\hline
\end{tabular}

Figure 1.6: Tiered Pricing (Ontario) [4].

\section{5-Year Fixed vs. 6-Month RPP Rates}

The decision on whether to lock in users' RPP rates depends on their risk tolerance and the degree to which they feel rates are likely to increase in the future.

As can be seen from Figure 1.7, the following hydro rate chart is a sample. When the users lock in their hydro rate, they tend to pay their contract, but in the later years of their contract they tend to pay less than the variable rate.

Another factor to be considered is when users use most of their electricity. The highest (on peak) rate of 14 cents/kWh is from 7:00 AM to 11:00 AM and from 5:00 $\mathrm{PM}$ to 7:00 PM in winter. In the summer, the on peak rate is from 11:00 AM to 5:00 PM weekdays. If households are busy during these times, and they stay with the RPP, they will be using a lot of power at the peak rates. By switching to a fixed-rate plan, they are not penalized when using power during peak-demand periods. The chart below shows how your Smart Meter allows your utility to be charged a different rate at different times of the day, see Figure 1.8. 


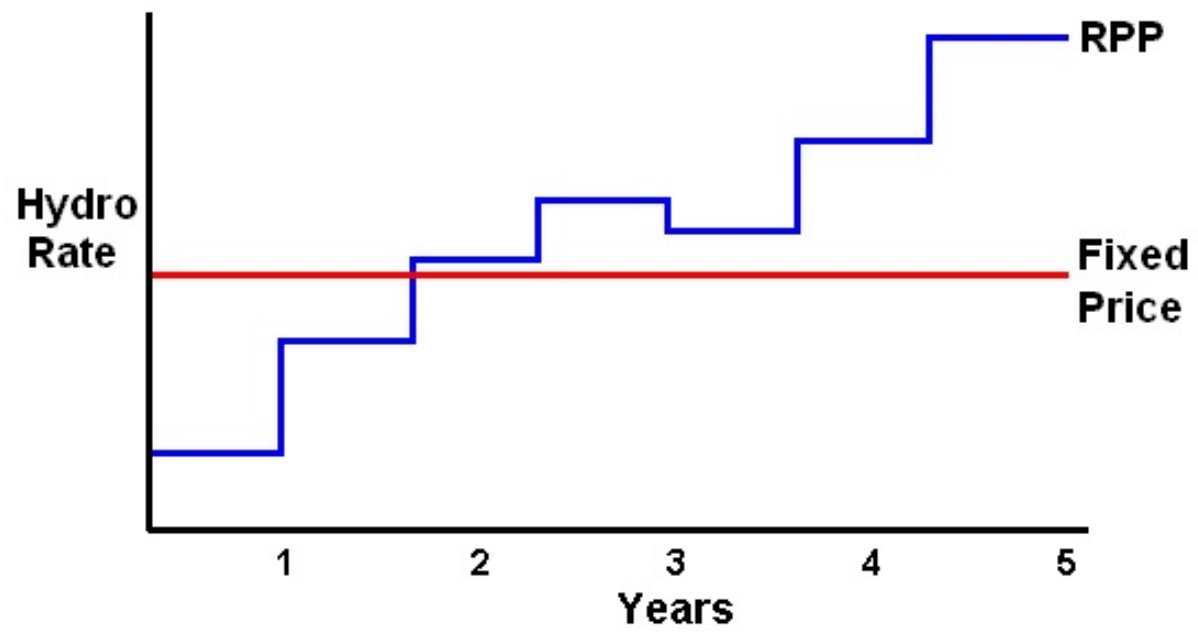

Figure 1.7: 5-Year Fixed vs. 6-Month RPP [4].

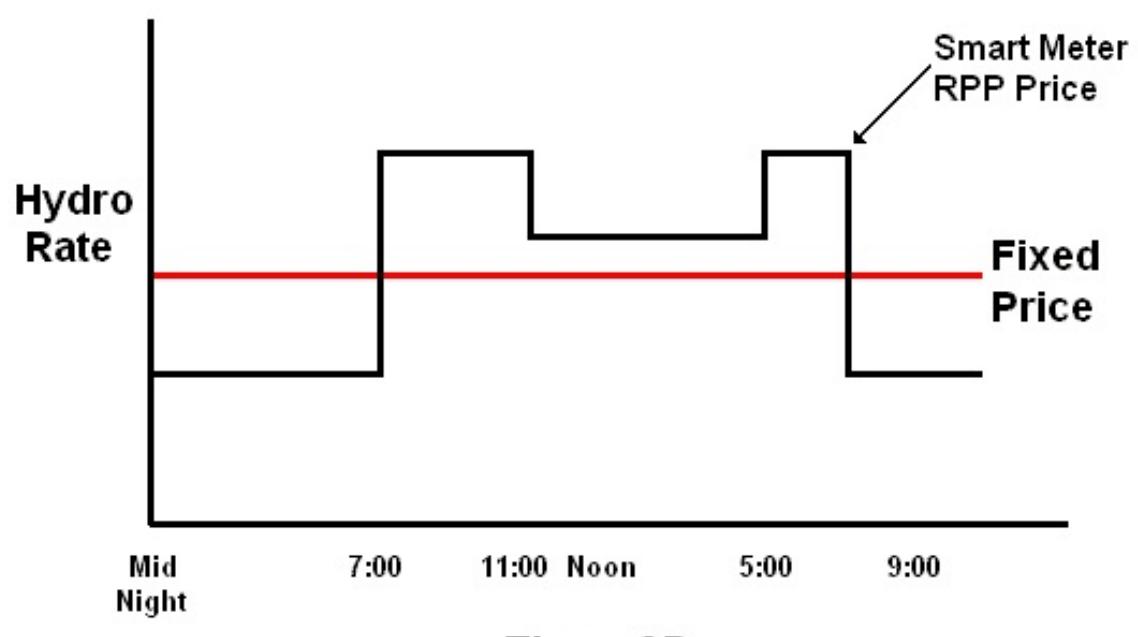

Time of Day

Figure 1.8: Constant vs. Fluctuating Rates [4]. 
With smart meters and time-of-use rates, distribution companies can provide detailed information on the customer web portal, MyHydroLink, itemizing how much electricity was consumed and when it was consumed. The bill also displays the three rate periods and the amount of consumption used within those periods. This is intended to encourage customers to shift ther consumption, where it is possible, to lower-cost times of the day and week, and to more actively manage their electricity usage. The benefit of using a smart meter is that when Ontario electricity consumers shift their usage to off-peak hours, it reduces peak demand on the provincial electricity system. Over the longer term, lower peak demand will mean a reduced need for new generating, transmission and distribution infrastructure, lowering costs for all Ontarians. In addition, a reduction in peak demands means that the province can also reduce its use of carbon dioxide emitting generators that are called on when demand is high, lowering greenhouse gas emissions. Smart meter data also provides comprehensive, detailed information for electricity system planning, allowing planners to identify where future generation, transmission and distribution investments are required. Smart meters also help LDCs identify power theft and respond to meter failures and outages more quickly, and they help LDCs identify greater operational efficiencies in local distribution system management. This efficiency lowers the electricity costs for customers. The infrastructure of a smart grid is similar to a telephone or Internet connection.

In our model, we use a dynamic pricing model based on the real and instant data from the HydroOttawa web page. This method is more reliable than the three rate period, because the model that is still in use in Ottawa is partly dynamic, rather than fully dynamic. Users are encouraged to use their power in a low-cost time, but this can lead to high consumption of energy in the low-cost time period. However, with dynamic pricing, user will see instant changes in price. This method increases 
the awareness of saving money and energy.

\subsubsection{Power Line Communications (PLCs)}

PLC uses the power feeder line as communication media in Figure 1.9. The first generation ripple control systems provide one-way communications, in which centralized load control and peak shaping have been performed for many years. The European standards body CENELEC restricted the use of frequencies between $3 \mathrm{kHz}$ and 95 $\mathrm{kHz}$ for two-way communications for electricity distributor use. A number of second generation PLC systems with low data rates were proposed in the 1990s, and AMR systems have been deployed based on this technology. Third generation systems based on OFDM with much higher data rates are currently being developed and deployed for smart grids, distribution automation and advanced metering management [24]. When the smart grid was developed, the importance of PLC and distribution networks became an important technology for transmitting the information between users and utility companies. To provide service between users and utility companies, the PLC design has to be improved with reliable data rates. Understanding PLC parameters are vital for two-way communication for the sake of high data rates and efficiency of the system. The main thing influencing the reliable communication of high-speed data on power lines is the attenuation of the high-frequency signal, which exhibits more obviously in the branch of power lines. It is almost impossible to use the frequency range of 10 to $20 \mathrm{MHz}$ for the reliable communications from the distributing transformer to the user, so it must be solved with the aid of repeaters and modulation schemes. 


\section{PLC : Power Line Communication}

Power line as Physical media for communications

\section{$\checkmark$ AMR (Automatic Meter Reading)}

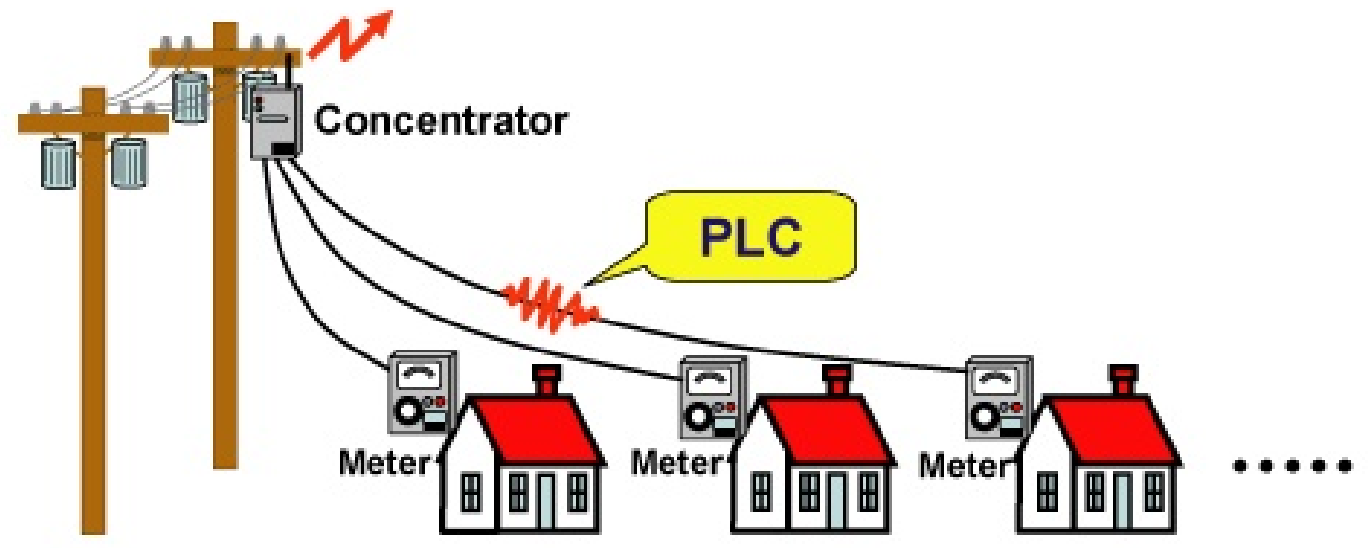

\section{$\diamond$ Home Network}

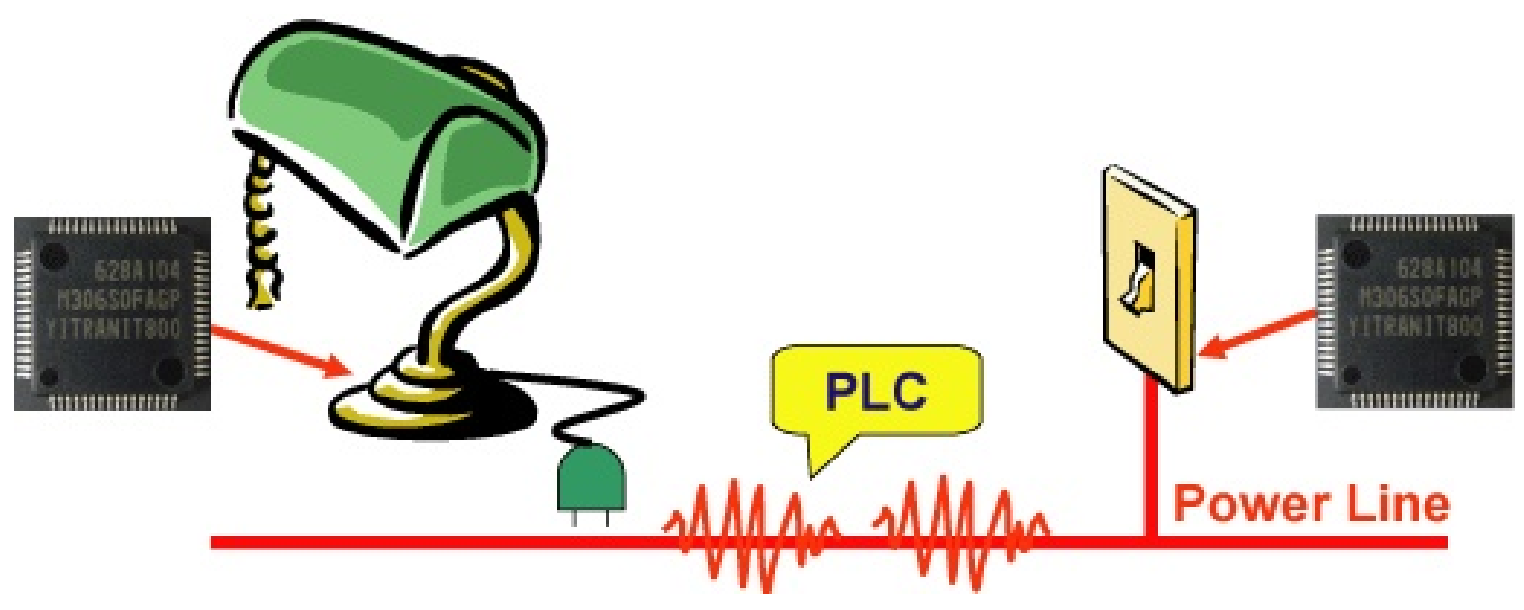

Figure 1.9: A Typical PLC Model [5]. 


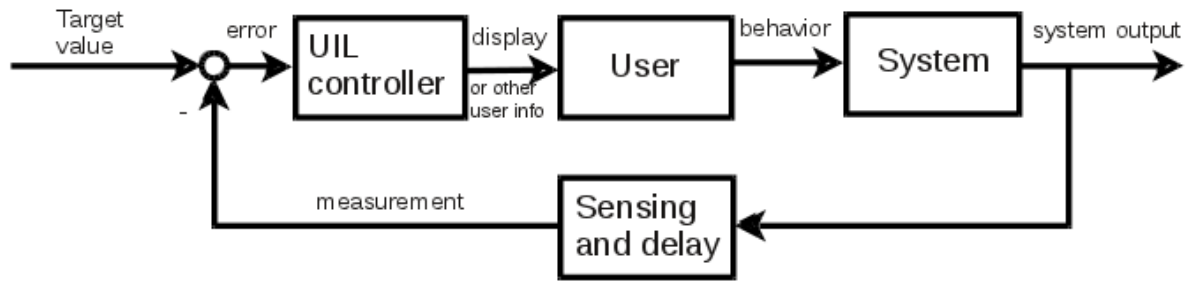

Figure 1.10: User-in-the-Loop (UIL) Model [6].

\subsection{UIL Definition and its Properties}

The idea of User in the Loop (UIL) is pointed out for users' "wireless behaviour" and aimed to control their behaviours to increase the efficiency of wireless data resources without limiting bandwidth (BW) [25]. Dr. Rainer Schoenen explores this idea in his paper, where he proposed that balancing or almost balancing the demand and supply will increase the efficiency of using wireless resources. In his paper [26], he focuses on controlling the demand side control; he pointed out that the control model is divided into two parts: Spatial control that users are encouraged to change their location to get higher data and avoid data traffic congestion [27], [28]. In temporal control, users are supposed to postpone their current data demand in case of congestion [29]. When users take any of these control models to avoid congestion, they are incentivized from the reduction on their monthly bill, bonus points, increase in the speed of data. etc. Incentives are based on instant data (i.e. they are dynamic) to ensure that the user reduces his or her usage. In Dr. Schoenen's public survey on how people react by changing their location or postponing the usage of their data, Dr. Schoenen indicates that people react positively towards these two control models, see Figure 1.10. 


\subsection{ZigBee Communications}

\subsection{ZigBee Physical Layer}

ZigBee communications is open to global wireless standards to provide the foundation for the Internet of Things by enabling simple and smart objects to work together, improving comfort and efficiency in everyday life. ZigBee is developed as an open global standard to address the unique needs of low-cost, low-power wireless M2M networks. The ZigBee standard operates on the IEEE 802.15.4 physical radio specification and operates in unlicensed bands including $2.4 \mathrm{GHz}, 900 \mathrm{MHz}$ and $868 \mathrm{MHz}{ }^{1}$.

\section{IEEE 802.15.4 specification}

- 802: networking group

- 15: wireless network

- 4: low data rate consuming less power

The 802.15.4 specification upon which the ZigBee stack operates gained ratification by the Institute of Electrical and Electronics Engineers (IEEE) in 2003. The specification is a packet-based radio protocol intended for low-cost, battery-operated devices. The protocol allows devices to communicate in a variety of network topologies and can have battery life lasting several years.

\subsubsection{Basics:}

- 868.0-868.6 MHz: Europe, allows one communication channel.

- 902-928 MHz: North America, up to ten channels (2003), extended to thirty (2006).

\footnotetext{
${ }^{1}$ In stead of ZegBee, Wi-fi can be used.
} 
- 2400-2483.5 MHz: Worldwide use, up to sixteen channels (2003, 2006).

\subsubsection{The ZigBee Protocol}

Member of the ZigBee alliance have created and ratified the ZigBee protocol. Over 300 leading semiconductor manufacturers, technology firms, OEMs and service companies comprise the ZigBee Alliance membership. The ZigBee protocol was designed to provide an easy-to-use wireless data solution characterized by secure, reliable wireless network architectures.

\subsubsection{Why Do We Need ZigBee Communications?}

The ZigBee protocol is designed to communicate data through hostile RF environments that are common in commercial and industrial applications, see Figure 1.12. ZigBee protocol features include:

- Support for multiple network topologies such as point-to-point, point-to-multi point and mesh networks.

- Low duty cycle provides long battery life

- Low latency

- Direct Sequence Spread Spectrum (DSSS)

- Up to 65,000 nodes per network

- 128-bit AES encryption for secure data connections

- Collision avoidance, retries and acknowledgment 


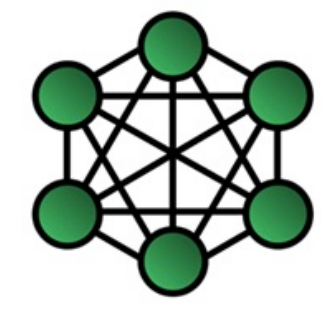

Figure 1.11: Mesh Networks

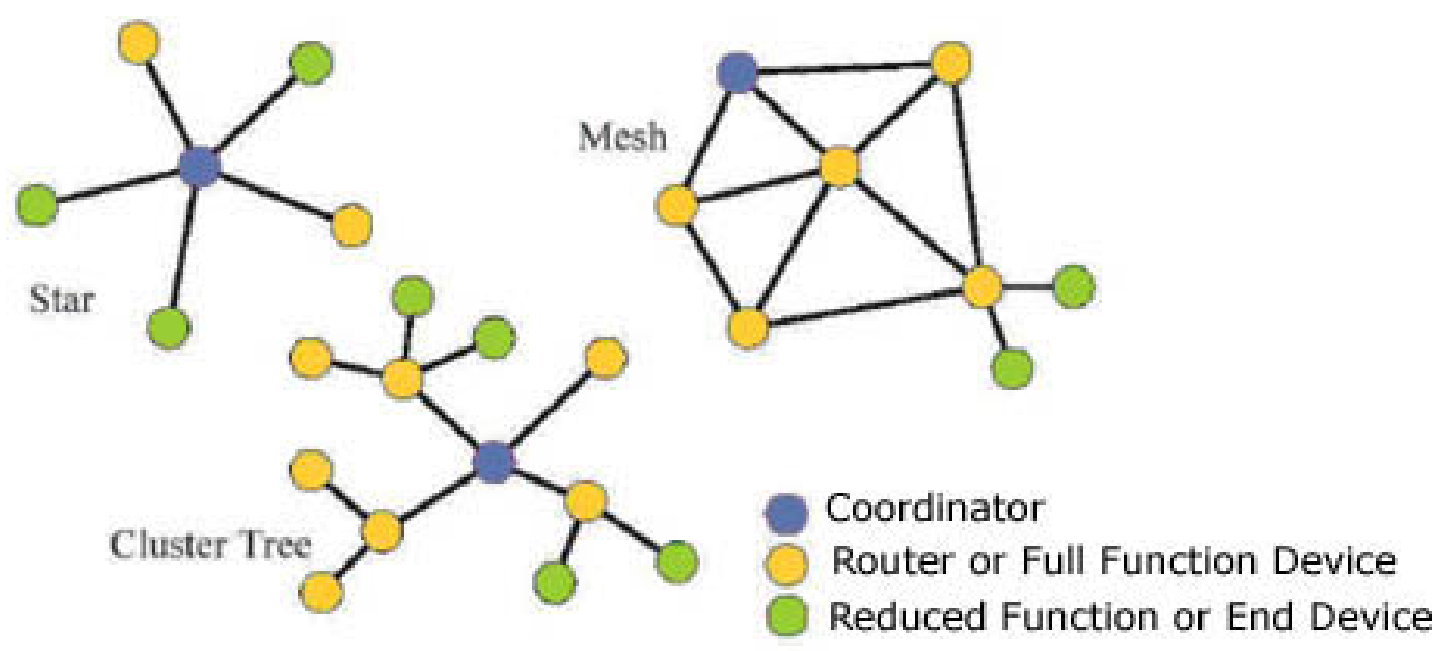

Figure 1.12: ZigBee Network [7]

\subsubsection{Mesh Networks}

A key component of the ZigBee protocol is the ability to support mesh networking. In a mesh network, nodes are interconnected with other nodes so that multiple pathways connect each node, see Figure 1.11. Connections between nodes are dynamically updated and optimized through a sophisticated, a built in mesh routing table.

Mesh networks are decentralized in nature; each node is capable of self-discovery on the network. Also, as nodes leave the network, the mesh topology allows the nodes to reconfigure routing paths based on the new network structure. The characteristics 
of mesh topology and ad-hoc routing provide greater stability in changing conditions or in the failure at single nodes.

\section{ZigBee Applications}

ZigBee enables broad-based deployment of wireless networks with low-cost, low-power solutions. It provides the ability to run for years on inexpensive batteries for a host of monitoring and control applications. Smart energy/smart grid, AMR, lighting controls, building automation systems, tank monitoring, HVAC control, medical devices and fleet applications are just some of the many spaces where ZigBee technology is making significant advancements, see Figure 1.13.

\section{Digi ZigBee Technology}

Digi is a member of the ZigBee Alliance. It has developed a wide range of networking solutions based on the ZigBee protocol. XBee and XBee-PRO modules and other XBee-enabled devices provide an easy-to-implement solution that provides functionality to connect to a wide variety of devices.

\section{Application Examples}

ZigBee is well suited for a wide range of control uses in just about any market. The Alliance has focused its standards development efforts around the commercial, residential, energy, consumer and industrial sectors. It has developed global standards for energy management and efficiency, home and building automation, health care and fitness, telecom and consumer electronics. Here are just a few examples of what the standards control: 


\begin{tabular}{|c|c|c|c|c|c|c|}
\hline \multicolumn{7}{|c|}{ Wireless Networking Technologies } \\
\hline Standard & $\begin{array}{c}\text { ZigBee } \\
\text { IEEE } \\
802.15 .4\end{array}$ & $\begin{array}{l}\text { Bluetooth } \\
\text { IEEE } \\
802.15 .1\end{array}$ & $\begin{array}{c}\text { UWB } \\
\text { IEEE } 802.15 .3 a \\
\text { (to be ratified) }\end{array}$ & $\begin{array}{l}\text { Wi-Fi } \\
\text { IEE } 802.11 \mathrm{a}, \\
\text { b, g }(\mathrm{n} \text { to be } \\
\text { ratified) }\end{array}$ & $\begin{array}{c}\text { LonWorks } \\
\text { EIA 709.1, 2, } 3\end{array}$ & $\begin{array}{l}\text { Proprietary } \\
\text { Proprietary }\end{array}$ \\
\hline $\begin{array}{r}\text { Industry } \\
\text { organizations }\end{array}$ & $\begin{array}{l}\text { ZigBee } \\
\text { Alliance }\end{array}$ & Bluetooth SIG & $\begin{array}{l}\text { UWB Forum } \\
\text { and WiMedia } \\
\text { Alliance }\end{array}$ & Wi-Fi Alliance & $\begin{array}{c}\text { LonMark } \\
\text { Interoperability } \\
\text { Association }\end{array}$ & $\mathrm{N} / \mathrm{A}$ \\
\hline Topology & $\begin{array}{c}\text { Mesh, } \\
\text { star, tree }\end{array}$ & Star & Star & Star & Medium-dependent & P2P, star, mesh \\
\hline $\begin{array}{r}R F \\
\text { frequency }\end{array}$ & $\begin{array}{c}868 / 915 \\
\mathrm{MHz} \\
2.4 \mathrm{GHz} \\
\end{array}$ & $2.4 \mathrm{GHz}$ & $\begin{array}{l}3.1 \text { to } 10.6 \mathrm{GHz} \\
\text { (U.S.) }\end{array}$ & $\begin{array}{l}2.4 \mathrm{GHz} \\
5.8 \mathrm{GHz}\end{array}$ & $\begin{array}{l}\text { N/A (wired } \\
\text { technology) }\end{array}$ & $\begin{array}{c}433 / 868 / 900 \\
\mathrm{MHz} \\
2 / 4 \mathrm{GHz}\end{array}$ \\
\hline Data rate & $\begin{array}{c}250 \\
\mathrm{kbits} / \mathrm{s}\end{array}$ & 723 kbits/s & $\begin{array}{c}110 \mathrm{Mbits} / \mathrm{s} \text { to } \\
1.6 \mathrm{Gbits} / \mathrm{s}\end{array}$ & $\begin{array}{c}11 \text { to } 105 \\
\text { Mbits/s }\end{array}$ & $\begin{array}{c}15 \mathrm{kbits} / \mathrm{s} \text { to } 10 \\
\text { Mbits/s }\end{array}$ & 10 to $250 \mathrm{kbits} / \mathrm{s}$ \\
\hline Range & $\begin{array}{c}10 \text { to } 300 \\
\mathrm{~m}\end{array}$ & $10 \mathrm{~m}$ & 4 to $20 \mathrm{~m}$ & 10 to $100 \mathrm{~m}$ & Medium-dependent & 10 to $70 \mathrm{~m}$ \\
\hline Power & Very low & Low & Low & High & Wired & Very low to low \\
\hline $\begin{array}{r}\text { Battery } \\
\text { operation } \\
\text { (life) } \\
\end{array}$ & $\begin{array}{l}\text { Alkaline } \\
\text { (months } \\
\text { to years) }\end{array}$ & $\begin{array}{c}\text { Rechargeable } \\
\text { (days to } \\
\text { weeks) }\end{array}$ & $\begin{array}{l}\text { Rechargeable } \\
\text { (hours to days) }\end{array}$ & $\begin{array}{l}\text { Rechargeable } \\
\text { (hours) }\end{array}$ & $\mathrm{N} / \mathrm{A}$ & $\begin{array}{c}\text { Alkaline } \\
\text { (months to years) }\end{array}$ \\
\hline Nodes & 65,000 & 8 & 128 & 32 & 32,000 & 100 to 1000 \\
\hline
\end{tabular}

Figure 1.13: Bluetooth vs. Wi-Fi vs. ZigBee vs. Others [8]

- Demand Response

- Advanced Metering Infrastructure

- Automatic Meter Reading

- Lighting controls

- HVAC control

- Heating control

- Wireless smoke and CO detectors

- Home security

- Blind, drapery and shade controls

- Medical sensing and monitoring 
- Remote control of home entertainment systems

- Indoor location sensing

- Advertising on mobile devices

The main trend in ZigBee development is improving power management and stack interoperability. These features are called Smart Energy 2.0 (effort was launched in 2008 to offer IP-based HAN functionality).

\subsubsection{The Concept of Open-Loop Control (OLC) and Closed- Loop Control (CLC)}

An open-loop controller, also called a non-feedback controller, is a type of controller that computes its input into a system using only the current state and its model of the system. The open-loop system is also called manual control system, see Figure 1.14 .

A characteristic of the open-loop controller is that it does not use feedback to determine if its output has achieved the desired goal of the input. Thus the system does not observe the output of the processes that it is controlling. Consequently, a true open-loop system cannot engage in machine learning and also cannot correct any potential errors. It also compensate for disturbances in the system.

An open-loop controller is often used in simple processes because of its simplicity and low cost, especially in systems where feedback is not critical. A typical example would be a conventional washing machine, for which the length of machine wash time is entirely dependent on the judgement and estimation of the human operator. In a smart home, the open-loop controller can be used in some cases. The users can adjust themselves to operate their smart appliances or by not tracking the instant price. The 


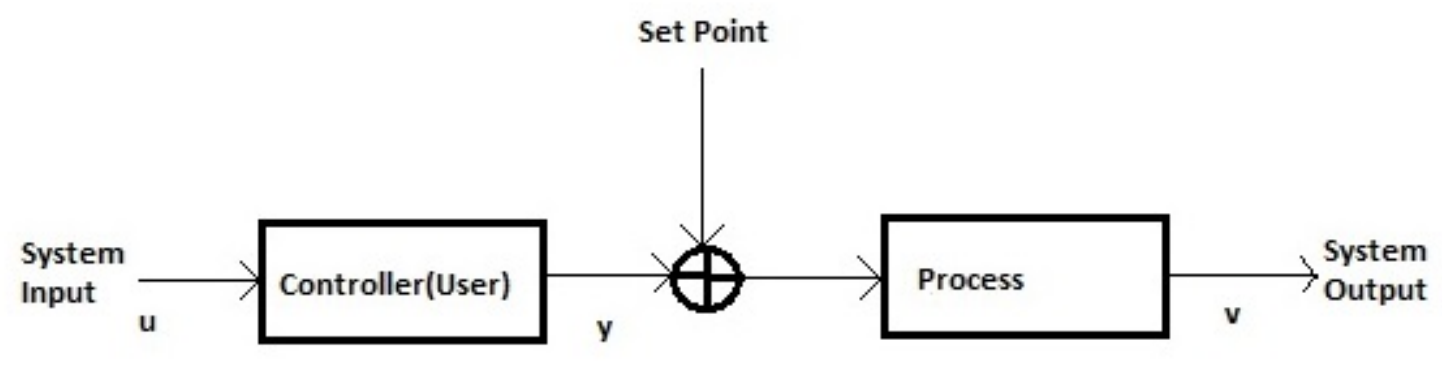

Figure 1.14: Open-Loop Model

instant price gives an assumption about what total energy consumption will be. On the surface, open-loop seems to be informatics for households. Households tent to observe only the current value of electricity and make a decision. The participation of the users in energy consumption is called user-in-the-loop (UIL). Since the system is an open-loop, it is called UIOL.

\section{Advantages Of Open-Loop Control System}

- Simple in construction and design.

- Economical.

- Easy to maintain.

- Generally stable.

- Convenient to use, as output is difficult to measure.

A Closed-loop Control System, also known as a feedback control system, is a control system that uses the concept of an open-loop system as its forward path, but has one or more feedback loops (hence its name) or paths between its output and its 


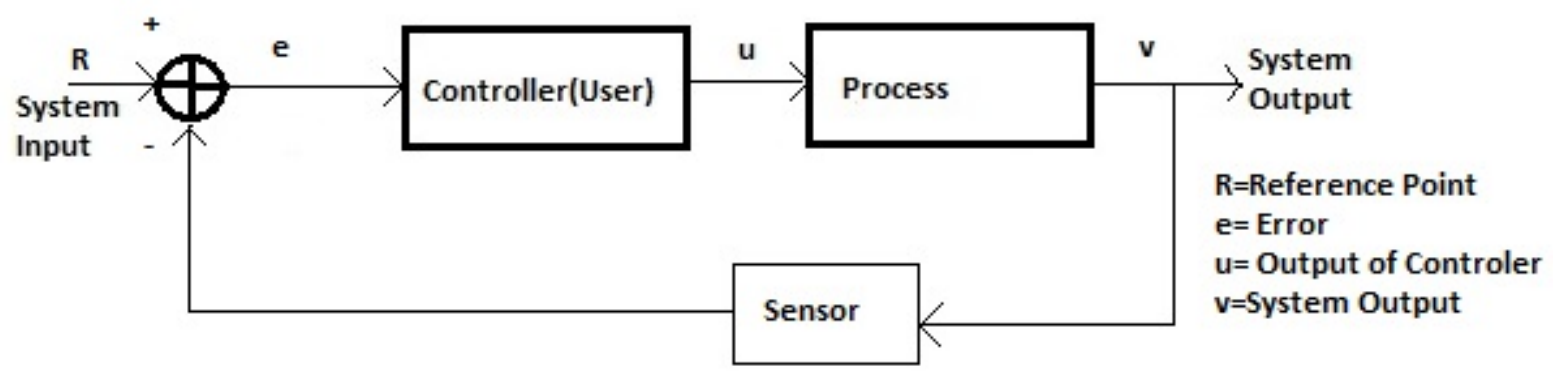

Figure 1.15: Closed-Loop Control Model

input. The reference to feedback simply means that some portion of the output is returned back to the input, to form part of the system's excitation.

Closed-loop systems are designed to automatically achieve and maintain the desired output condition by comparing it with the actual condition. It does this by generating an error signal which is the difference between the output and the reference input. In other words, a closed-loop system is a fully automatic control system with its control action is dependent on the output in some way.

\section{The Advantages of Closed-loop Control}

- Reduce errors by automatically adjusting the system's input.

- Improve stability of an unstable system.

- Increase or reduce the systems sensitivity.

- Enhance robustness against external disturbances to the process.

- Produce a reliable and repeatable performance.

- Bandwidth range is large. 
While a good closed-loop system can have many advantages over an open-loop control system, its main disadvantage is that in order to provide the required amount of control, a closed-loop system must be more complex by having one or more feedback paths. Also, if the gain of the controller is too sensitive to changes in its input commands or signals, it can become unstable and start to oscillate as the controller tries to over-correct itself, and eventually risking it breaking. So we need to tell the system how we want it to behave within some predefined limits.

Our system model is both an open-loop and closed-loop for UIL systems. In an open-loop system the users are not supposed to give any feedback to the utility company about their energy usage. However, the users can affect the whole system by delaying their usage. Users are important active parameters that have initial impact on the energy and money saving model. By looking at the users' demands, the utility company can adjust the production accordingly. Users make decisions after energy generation beyond a short time; this may not be effective for energy saving purposes. On the other hand, there is still something to do for demand shaping ideas. The average energy usage of almost all types of appliances are similar. Assuming that a washing machine usual time is 2 hours, utility companies can estimate how much energy is using and how long it will continue. This is a classic approach, however when it comes to millions of people's applications, it is difficult to apply. Likewise, most people prefer to use their appliances in their free time when the electricity price may harm their budget.

An approach to change users' decisions in terms of using their electricity is to encourage them to delay their usage to later time when the electricity price is lower than the average. Users will get some benefits by doing this.

Closed-loop model is a more efficient way to achieve an energy saving goal. In this way, users instantly connect to their utility company to recover the instant price. 
By doing this, users are encouraged to manage their energy and use their appliances during the time when the price is low. The system repeats itself to update the electricity price instantly and give a quick and correct feedback to users. Users will get benefits by doing this as well.

\subsubsection{Problem Statement}

- Why do we change pricing models from fixed to current?

Our goal is to encourage end users to be an important parameter of saving energy and money by using UIL method. For the sake of this idea users are supposed to postpone or delay their demand to a latter time. To do that there must be a time that the base price of electricity is relatively lower than the other times. In the fixed pricing model, we have a stable base price in the whole year; therefore, UIL method cannot work in this pricing model. In the current model, we have a partial dynamic pricing method; therefore, UIL concept can be applied here. In the half of the day, the electricity base prices are under the average. For this reason, to help users to save energy and money, current pricing model can be used in stead of fixed pricing model.

\section{- Why do we need a dynamic pricing model?}

Current pricing model works better than the fixed pricing model. However, to get precise results, we need a fully dynamic pricing model. By doing this, users turn on their appliances during the low-peak interval. Users' demands remain same, but they shift the demands to a low peak hour. When the number of users increase, the energy saving decrease due to the increment in the average base price. 
- Why do we use 5 minutes cycle?

To say our system is a full dynamic system and supply changes very fast due do the fast demand response, we have to reduce the interval as much as we can. For this purposes, we take 5 minutes interval. In the literature, this interval is taken 1 hour but it may not give a precise results in terms of saving [30], [31].

\section{- Why UIL is more suitable for $5 \mathrm{G}$ instead of $4 \mathrm{G}$ ?}

UIL method can work under in $4 \mathrm{G}$ and $5 \mathrm{G}$ technologies. Since $4 \mathrm{G}$ is already existed and mature technology, setting up UIL method cannot be \%100 percent applicable in this technology. Instead of 4G, 5G offers a more application based IoTs technology which may involve smart grid communications. Besides, 5G as the first network designed to be scalable, versatile, and energy smart for the hyper-connected internet of everything world. 5G networks will be faster and a lot smarter. It is assumed that IoTs generate massive data which cause congestion in network. $5 \mathrm{G}$ may deal with this congestion by offering heterogeneous networks [32].

\section{- Why do we use UIL method?}

UIL method first used for networks and efficient results. We used the same idea for energy grid to contribute in saving energy and money.

\section{- Problem we have to solve}

Regarding to above, we have to make the problem statement. We know that users try to save their energy and money by reducing their demand. This may work until a point, but it is not an effective way because the need for energy increases day by day. Without decreasing the demand, users can reduce their payment to energy by postponing their demand. Thanks to this method the total supply and demand will converge. When the supply and demand converged 
with each other, in the big picture, the total supply will be reduced (shaped based on the demand). In other words, utilities generate energy based on users demand.

\subsubsection{Contributions}

After all, we can say that by setting up such systems and using UIL method, users save both energy and money. These methods can be integrated to 5G technology easily. We developed a dynamic pricing model with UIL method. We have used existing technologies effectively such as ZegBee, smart meter, smart grid communications, cellular networks, etc.

\subsubsection{Thesis Organization}

In Chapter 1, we introduce the base technologies that we use in our system model. In Chapter 2, we summarized our system models and compared some existing technologies with our model. In addition we set up our ideas with different research areas. In Chapter 3, we built an open-loop control model. In this model, we compared 3 different pricing models with or without the UIL method.

In Chapter 4, we built a closed loop control model rather than open-loop control. In this model, we compared 3 different pricing models with or without the UIL method. In Chapter 5, we analysed the results and make some statistical assumptions based on these results. 


\section{Chapter 2}

\section{System Definition}

The systems we proposed are both open-loop and closed-loop. We have compared three type of pricing models are used in literature.

\subsection{Pricing Systems Comparisons}

\subsubsection{Fixed Pricing Model}

The fixed pricing model is still in use in various countries. In this system, the base pricing method is totally different than the current pricing systems. The base price remains stable during the whole year; the change in the price is only adjusted once a year based on the inflation rate, smart meter charge, and other similar factors. In other words, it does not matter how much energy the consumers try to save or thereby contributing to the system for environmentally friendly purposes; the base price will

remain the same. Moreover, this system is concrete; there is no short time control on the system on the end users side. Therefore, most of the time supply and demand do not converge as seen in Figure 2.1. Supply and demand indicates electricity energy in our system model. 

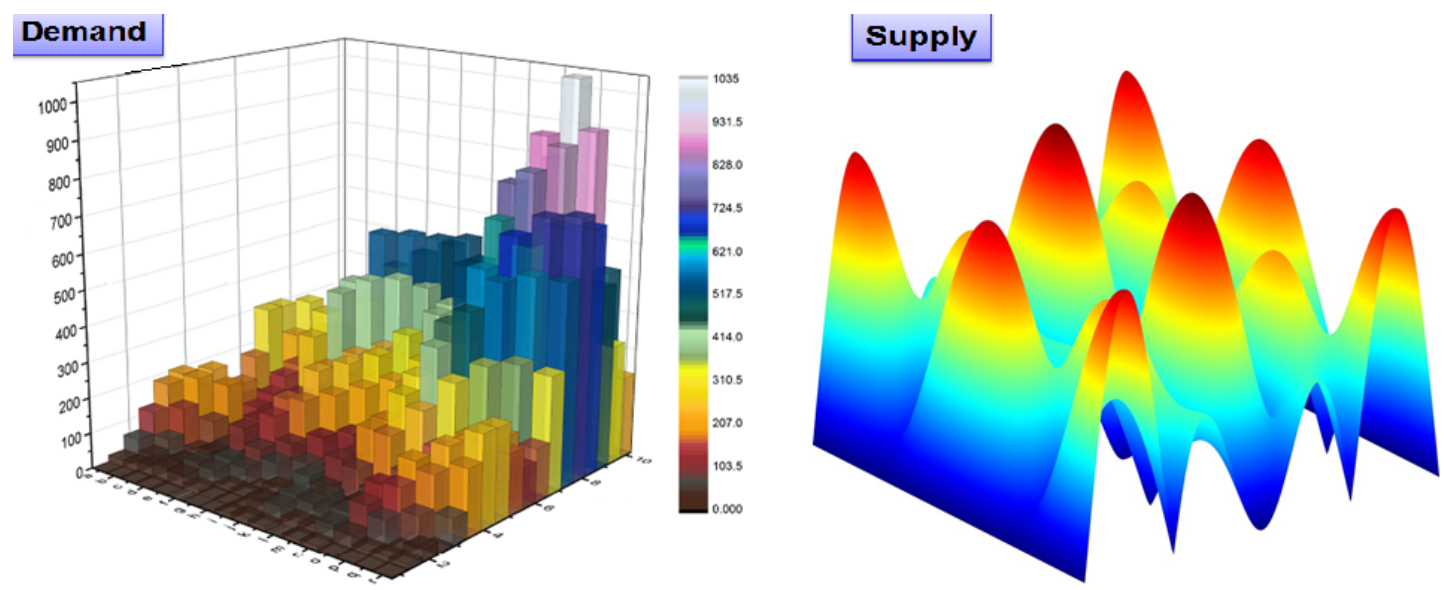

Figure 2.1: Demand and Supply

[8]

To meet the market demand, supply must be higher than demand in the most circumstances. Figure 2.2 shows the relation between supply and demand.

$P^{*}$ is the optimum price in which supply and demand exactly match with each other. $Q^{*}$ is quantity of the demand and supply where both are equal each other. Our goal is to provide this equilibrium in our system.

\subsubsection{Current Pricing Model (Partly Dynamic)}

Current pricing models are used in many countries such as Canada, United States and Turkey. This pricing model is partially dynamic, which is better than the fixed pricing model. If users have a smart meter installed in their house, the utility can charge varying rates throughout the day, as seen in Figure 2.3. These rates are divided into three separate bands known as off-peak, mid-peak and on-peak.

This pricing model gives ideas to the end users whether it would be suitable time to turn on their appliances based on peak hour estimates. As a result of this time 


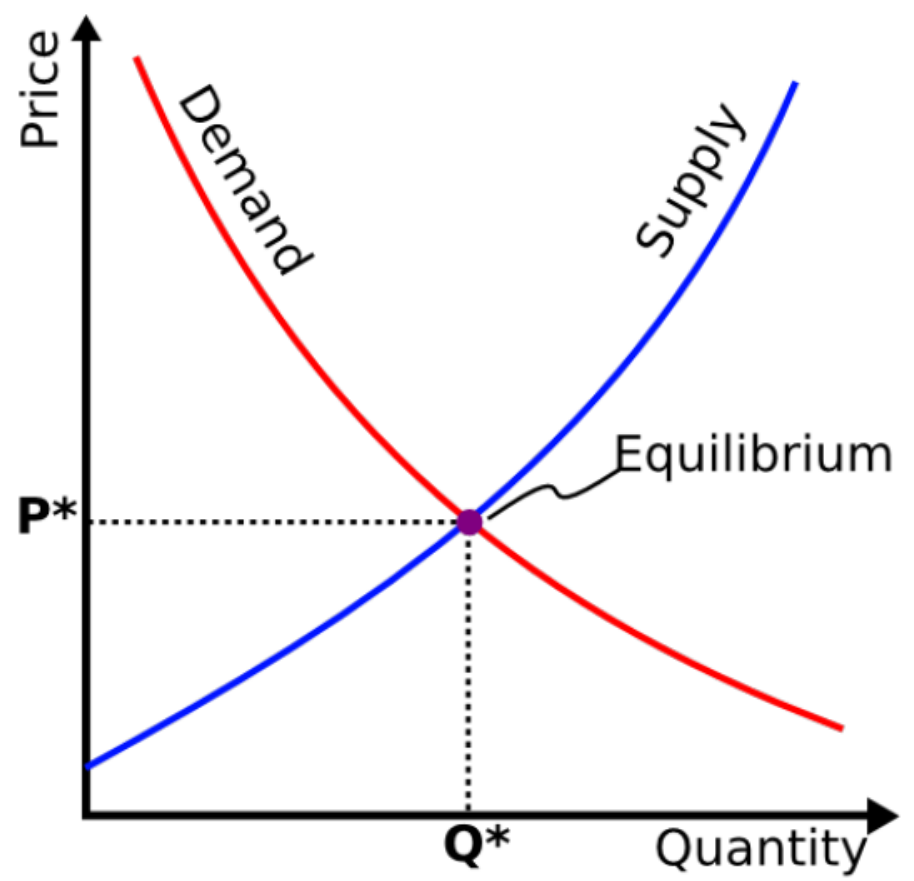

Figure 2.2: Supply-Demand-Price Relation [9]

distinction, users are able to estimate how much energy they consume and what will be the total price at end of each month. By knowing price ranges in advance, users can adjust their demand to save money or energy.

\subsubsection{Dynamic Pricing Model}

The dynamic pricing model promises a development to address the concern of finding an environmentally friendly solution of meeting energy demands so as to reduce customer monthly bills. This type of model is not currently in use. This system enables a fast communication between users and the utility companies. In each 5 minutes circle, users send feedback message to supplier to shape the supply. For this purpose, if end users shift their demand to a later time, they take the advantage of using electricity in off-peak hours without changing their total demand. In other words, 


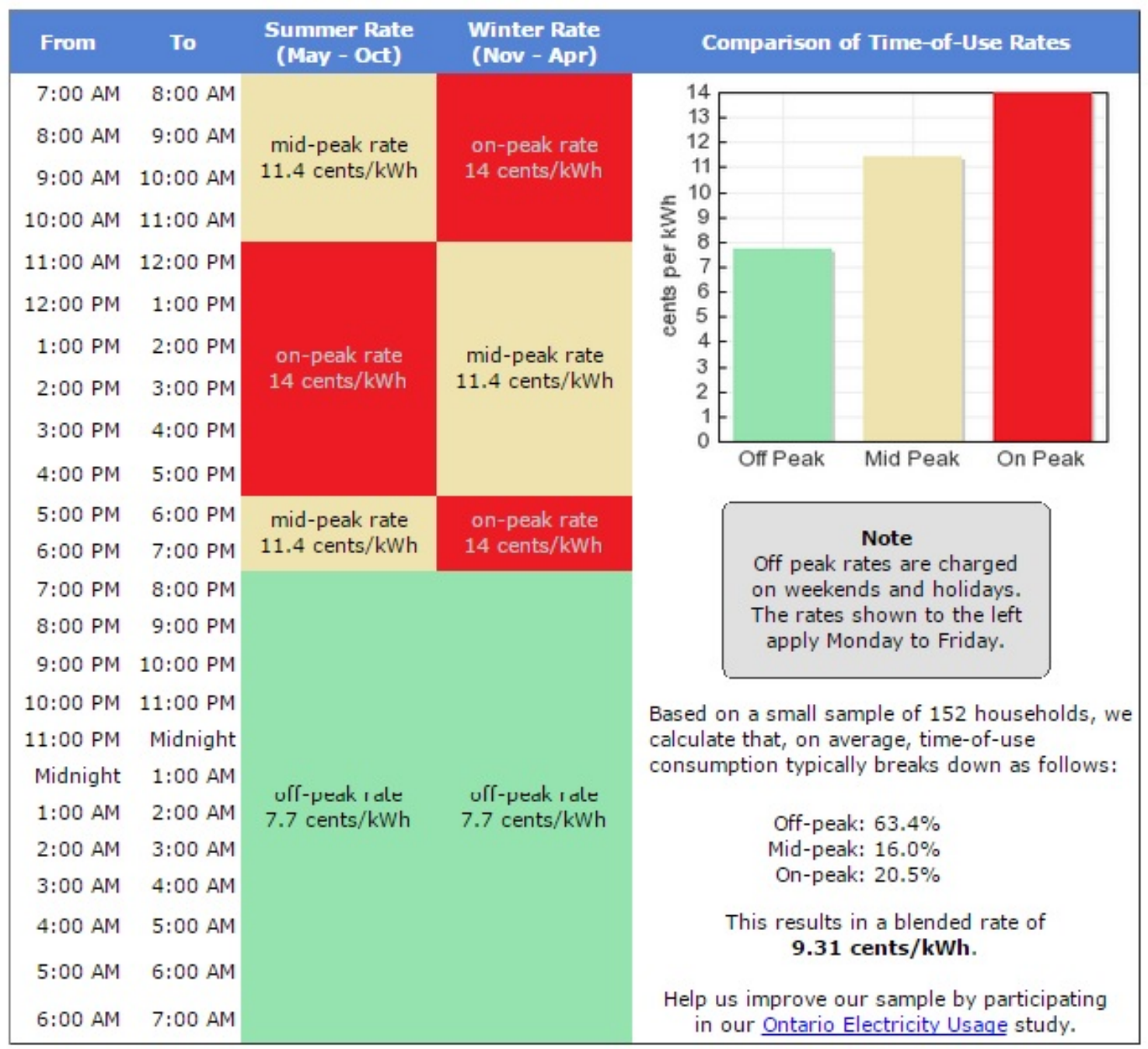

Figure 2.3: On-Off-Peak Hours for Ontario [10] 


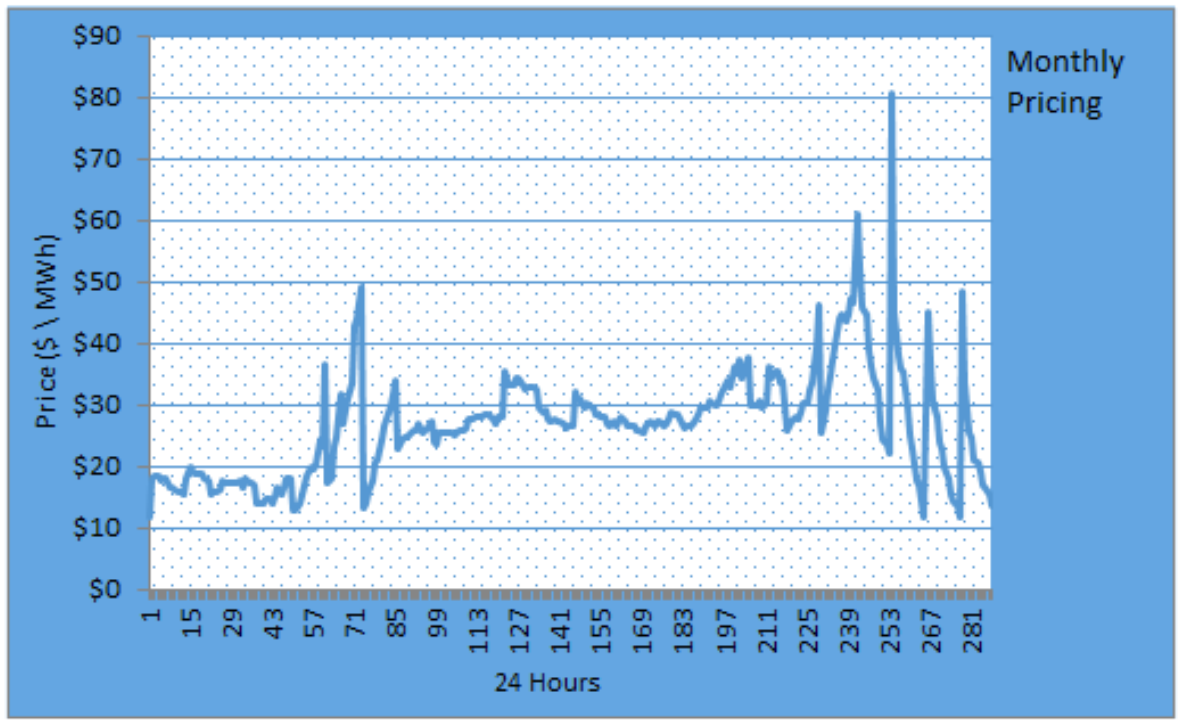

Figure 2.4: Dynamic Pricing for 5 Minutes Cycle

the idea is to reschedule end users' demand to a different time during a fixed interval based on dynamic energy prices during that interval.

Since we are assuming end users will avoid using their appliances during the red range where the price is on peak hour, as seen Figure 2.4, Figure 2.5, and Figure 2.6. The demand is not uniformly distributed any more. the usage in the red region is randomly distributed among the green range or at least in yellow range. This results in a super-possion distribution rather than a uniform distribution, see Figure 2.7 [11]. In other words, the demand is shaped based on the low cost of electricity, see Figure 2.8. Figure 2.8 illustrates that end users rarely turn on appliances on red zones. End users distribute their demand over time domain to extend the benefit of consuming energy at the certain time period. User's total demand remains same, but they postpone their consumption to a low cost time where they save money. 
Hourly Prices for February 23, 2015

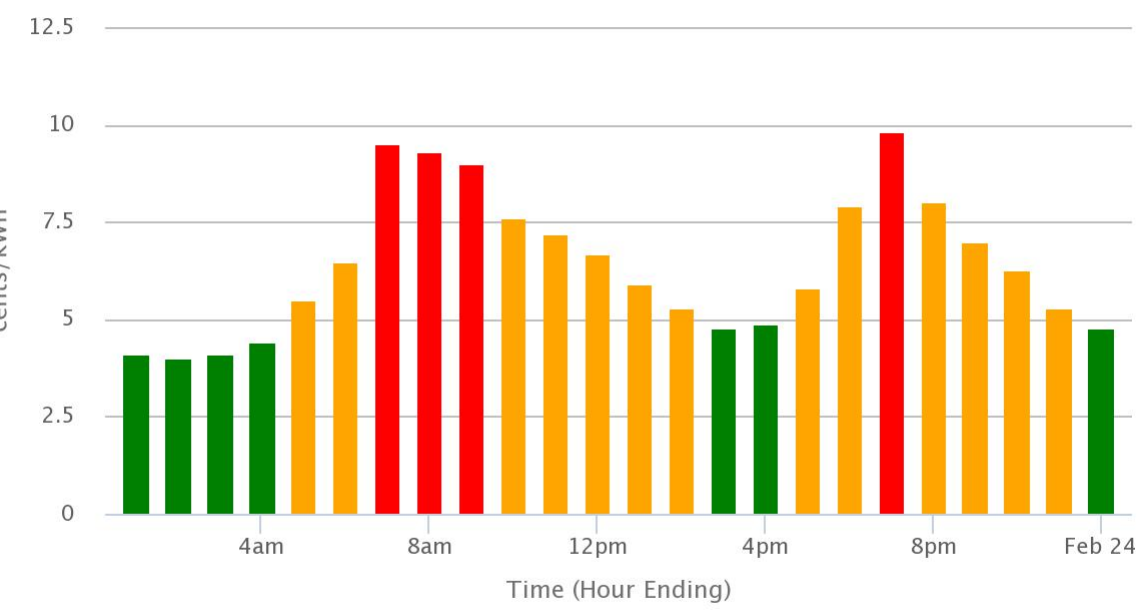

Price:

Figure 2.5: Green-Yellow-Red Regions for Illinois

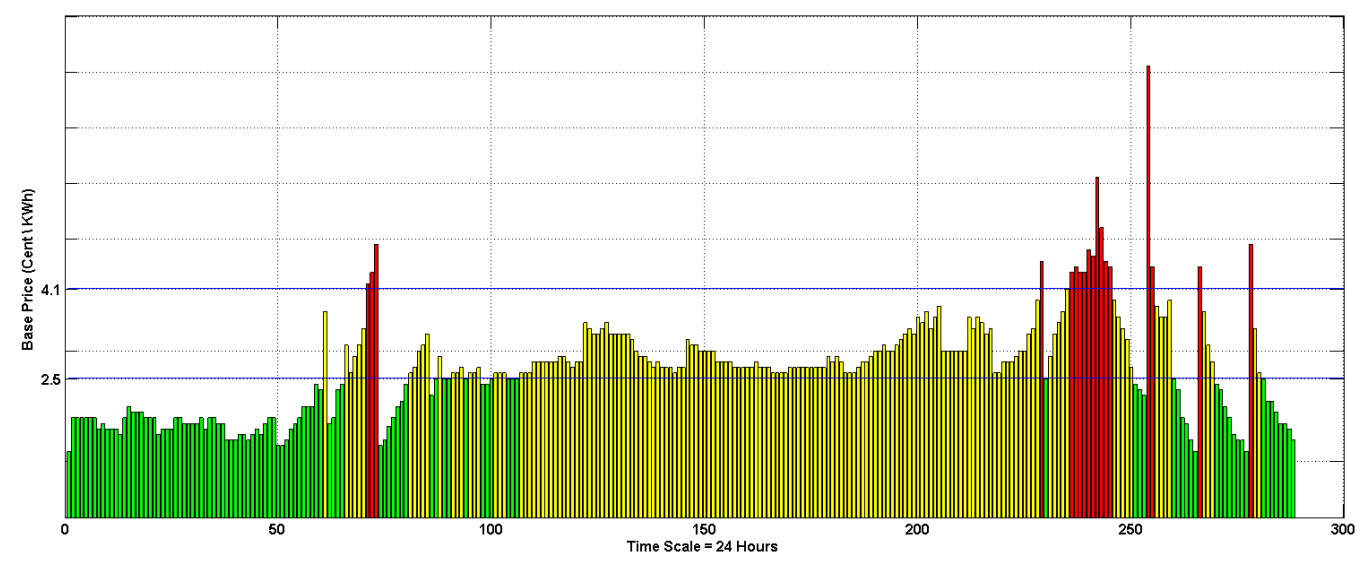

Figure 2.6: Green-Yellow-Red Regions for Ontario 

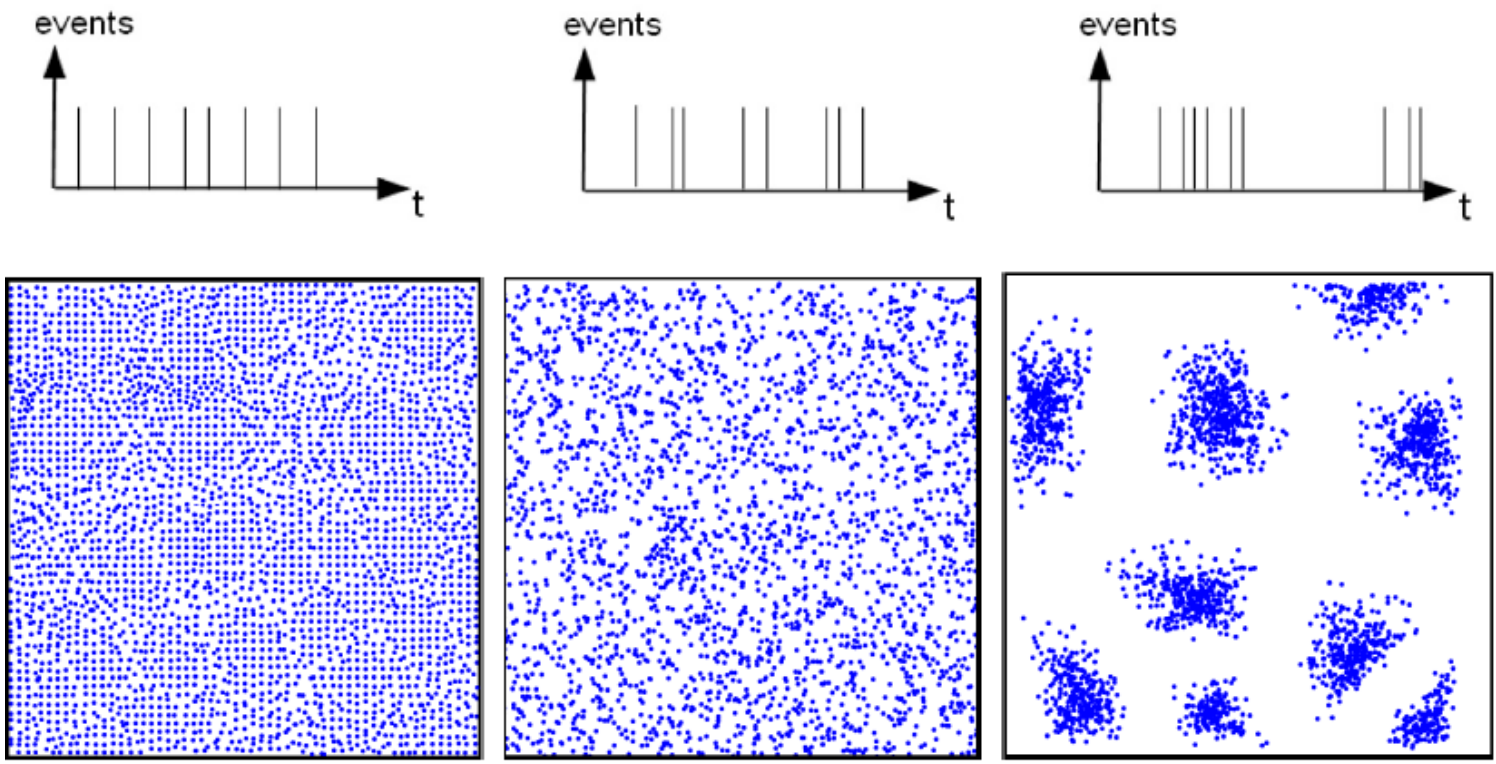

Figure 2.7: Sub-Poisson, Poisson, Super Poisson [11].

\subsection{Simulation Tools}

We have used the Simulink program for our system model comparisons, because Simulink is a block diagram environment for multi-domain simulation and modelbased design. It supports system-level design, simulation, automatic code generation, and continuous test and verification of embedded systems. Simulink provides a graphical editor, customizable block libraries, and solvers for modeling and simulating dynamic systems. It is integrated with MATLAB, enabling users to incorporate algorithms into models and export simulation results to MATLAB for further analysis. Simulink is widely used in control theory and digital signal processing for multi-domain simulation and model-based design. It is easy to implement control boxes instead of codes. However, there is more flexibility in using codes than in the control boxes in Simulink. Since Simulink is a visual program, it is easy to follow the processes. 

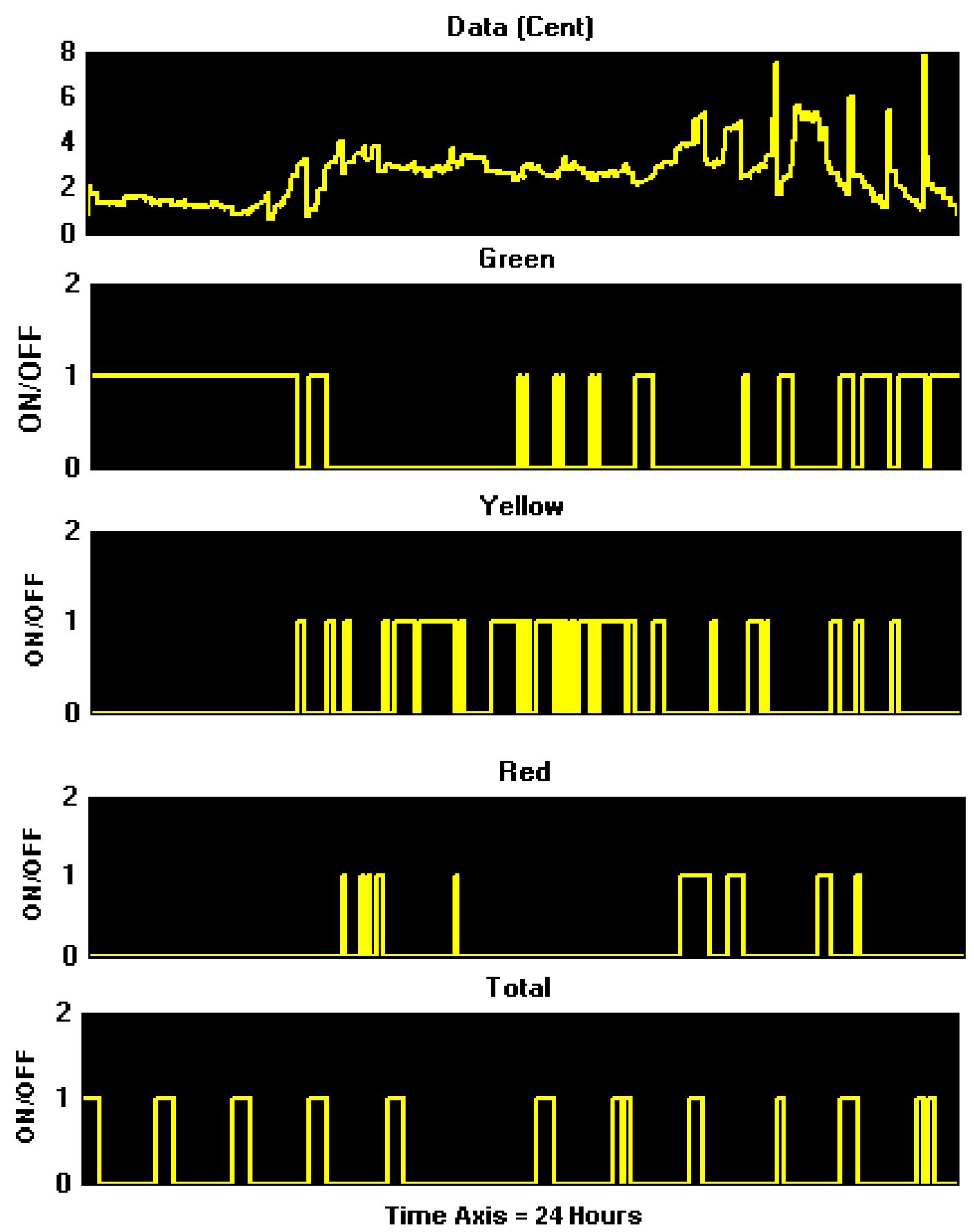

Figure 2.8: Air Conditioner 


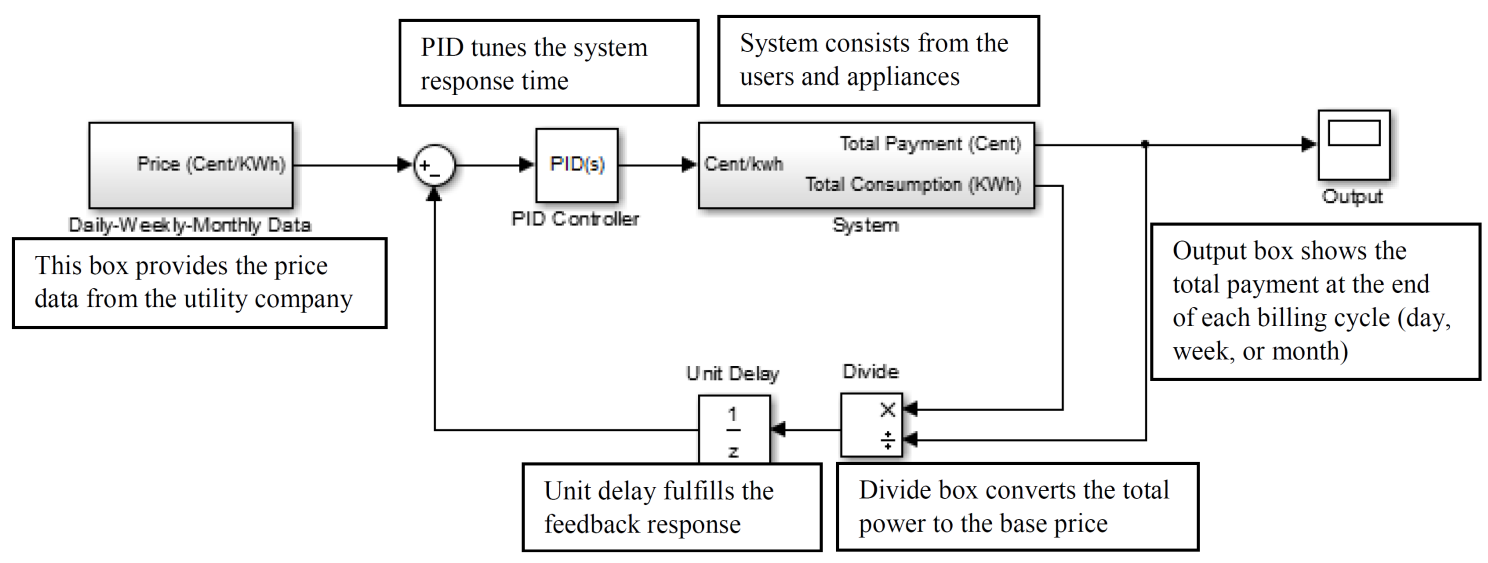

Figure 2.9: System Model

\subsection{System Model}

As seen in Figure 2.9, the system is a closed-loop model with PID controller. Figure 2.10 shows three different pricing models for understanding the UIL model better. These data are real and obtained from IESO website. As seen in Figure 2.11, we have 5 different systems and 50 users work in parallel. Random Selection box does a random selection among users, see Figure 2.12. In summary, our system consists of two main parameters: 1.Users which make decision, 2. Appliance in which users apply the low peak price, see Figure 2.13. Users are the enactor of the system. Their decisions are important to shape the demand, see Figure 2.14. Counting box counts the day where the UIL method is applied, see Figure 2.15. In the system, we have two types of appliances: 1. Shiftable, 2. Non-shiftable. In non-shiftable appliances, the user cannot change the demand time. However, the demand shaping method it can be 


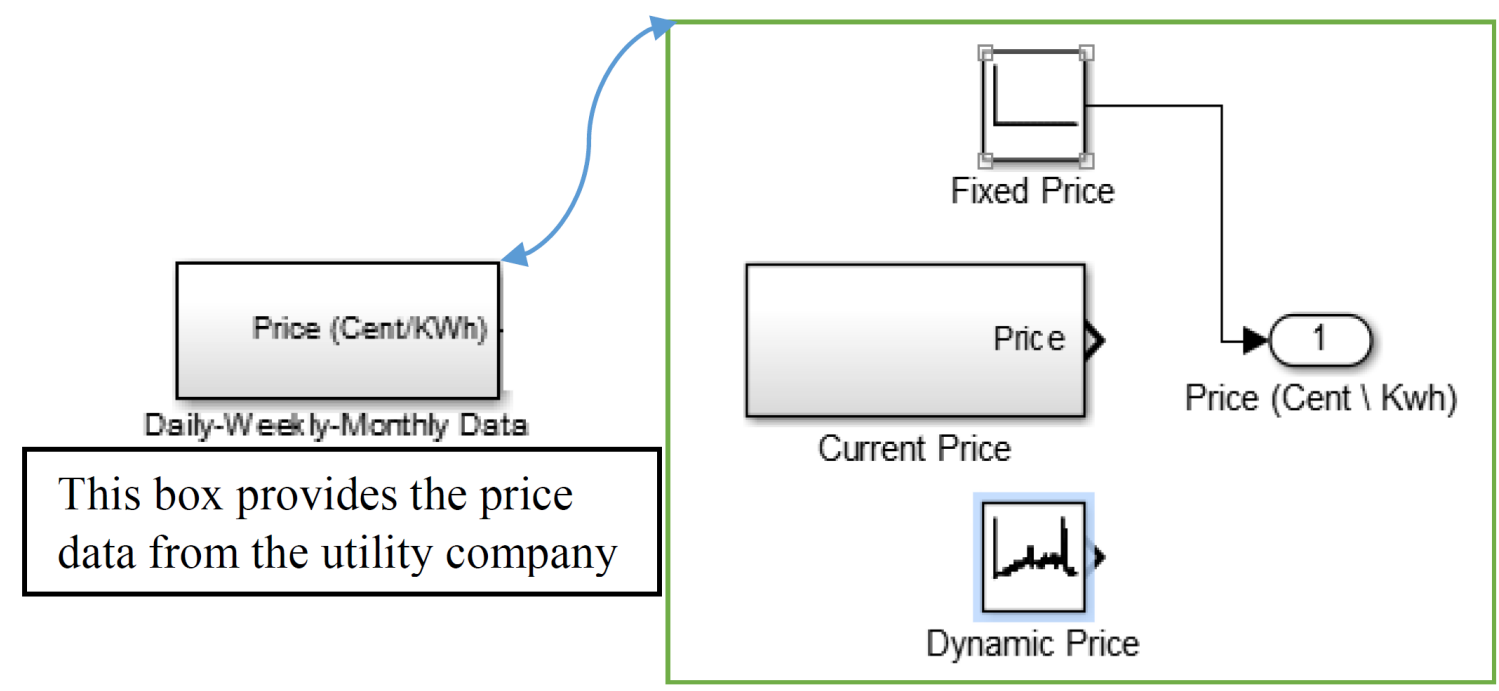

Figure 2.10: Price Added to the System from the Utility Company

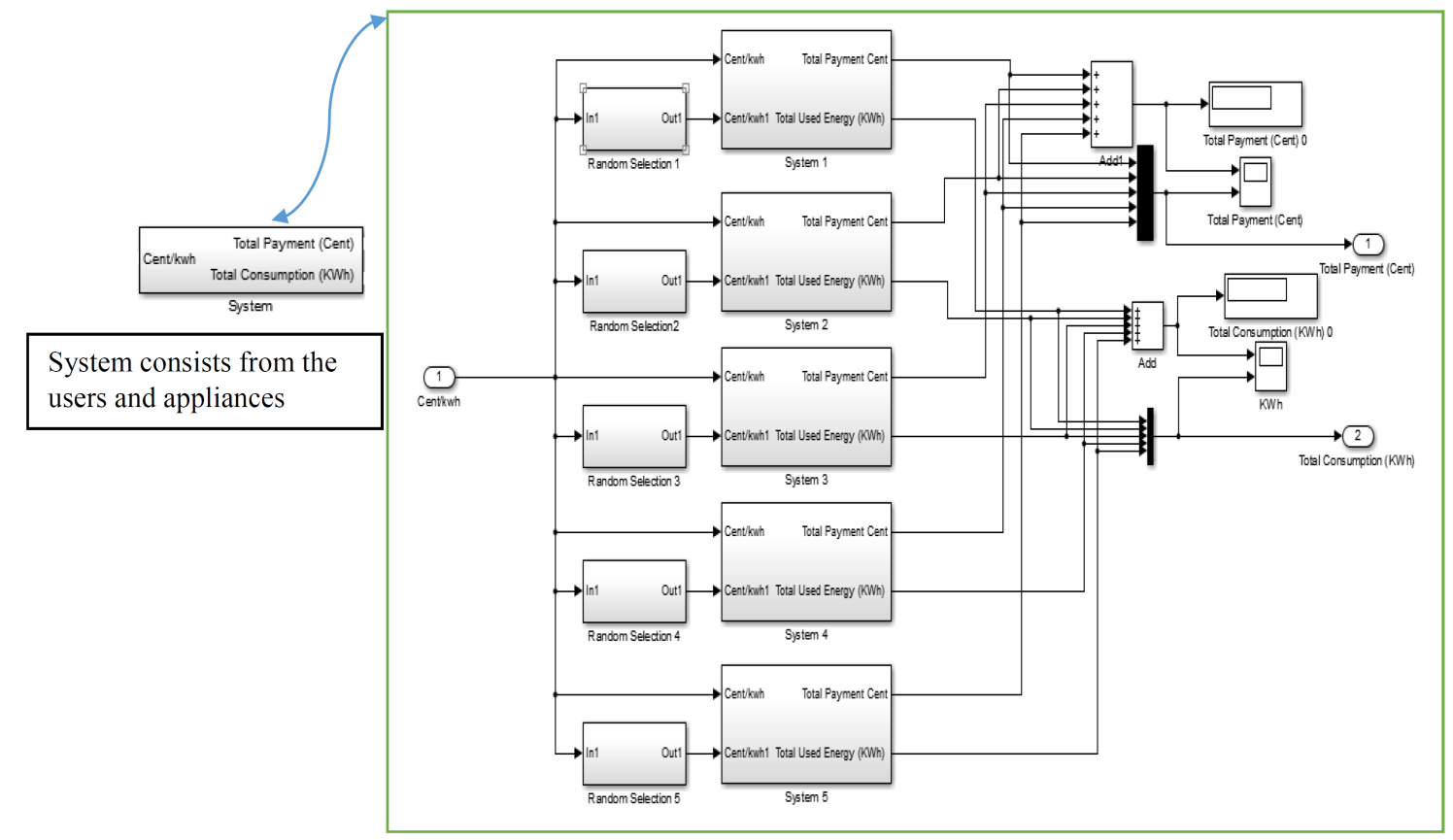

Figure 2.11: Backbone of the System Model 


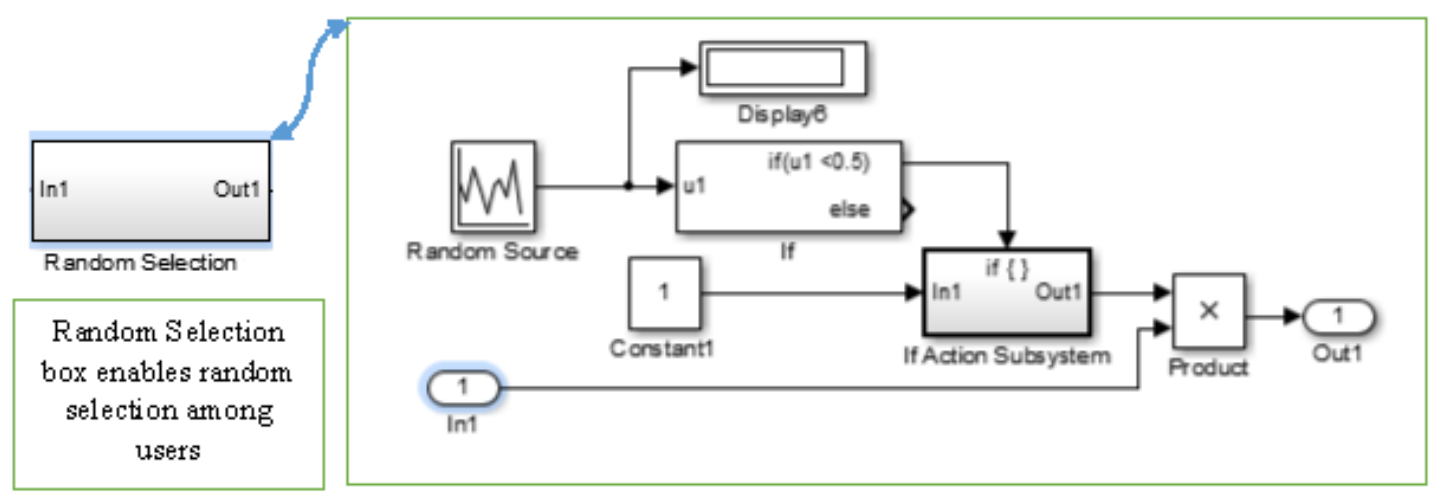

Figure 2.12: Random Selection Among Users

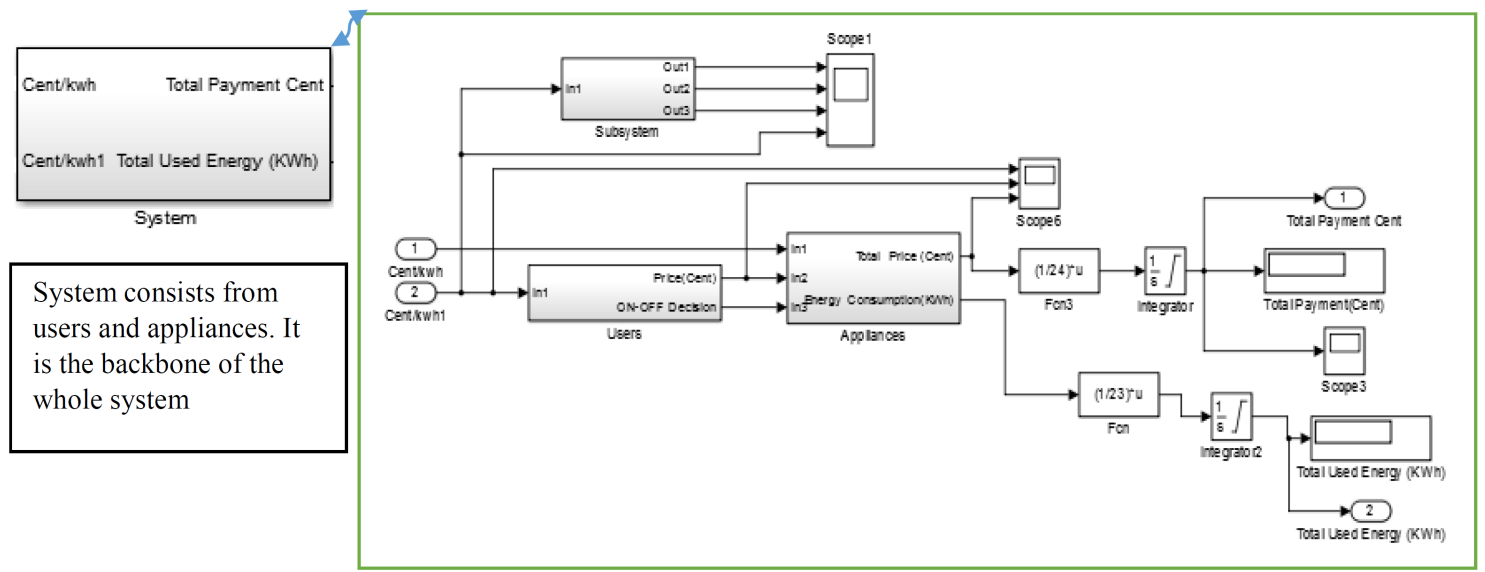

Figure 2.13: Main parameter of the System 


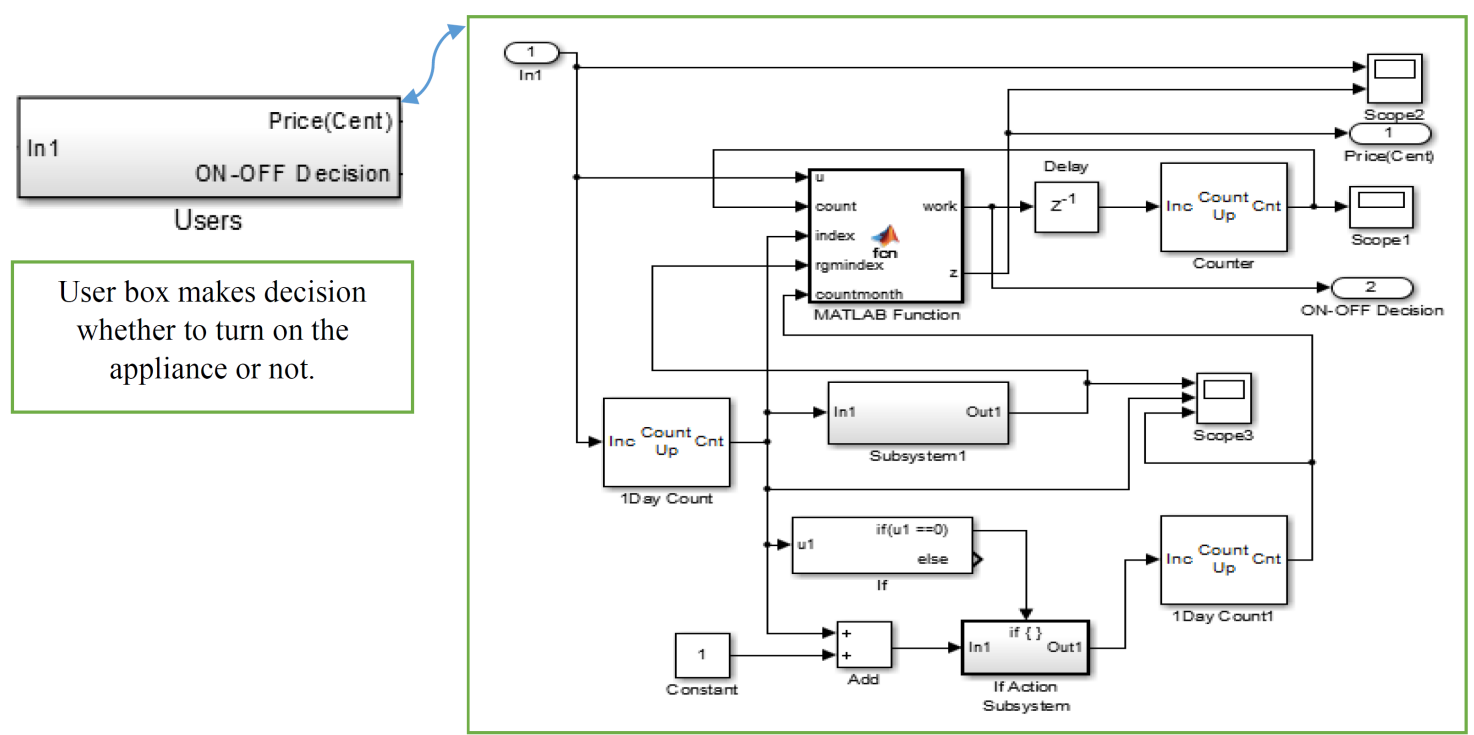

Figure 2.14: Main Parameter of the Users

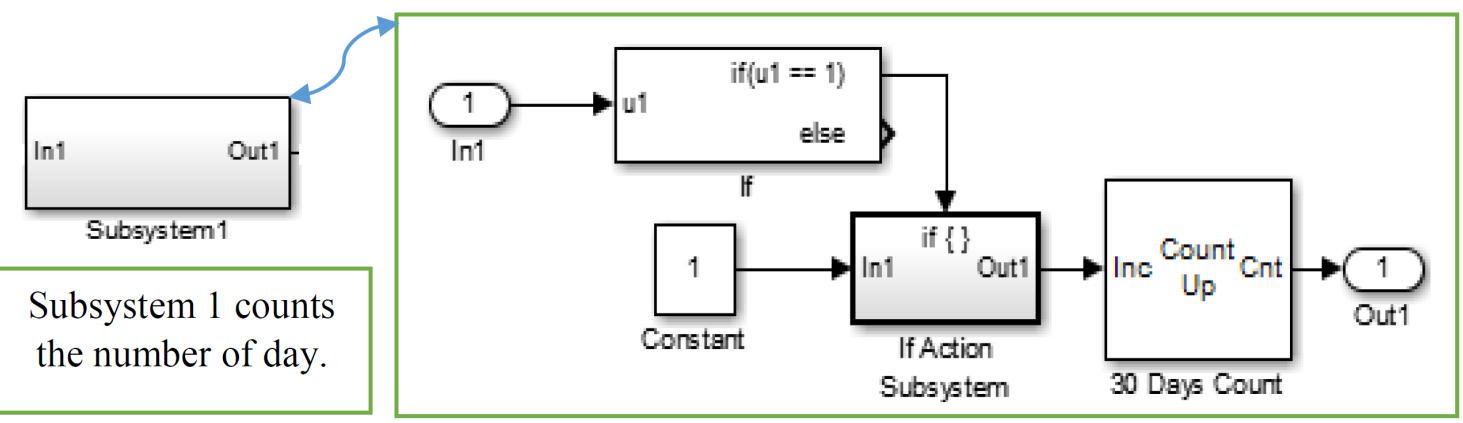

Figure 2.15: Counting Days with its Parameters 


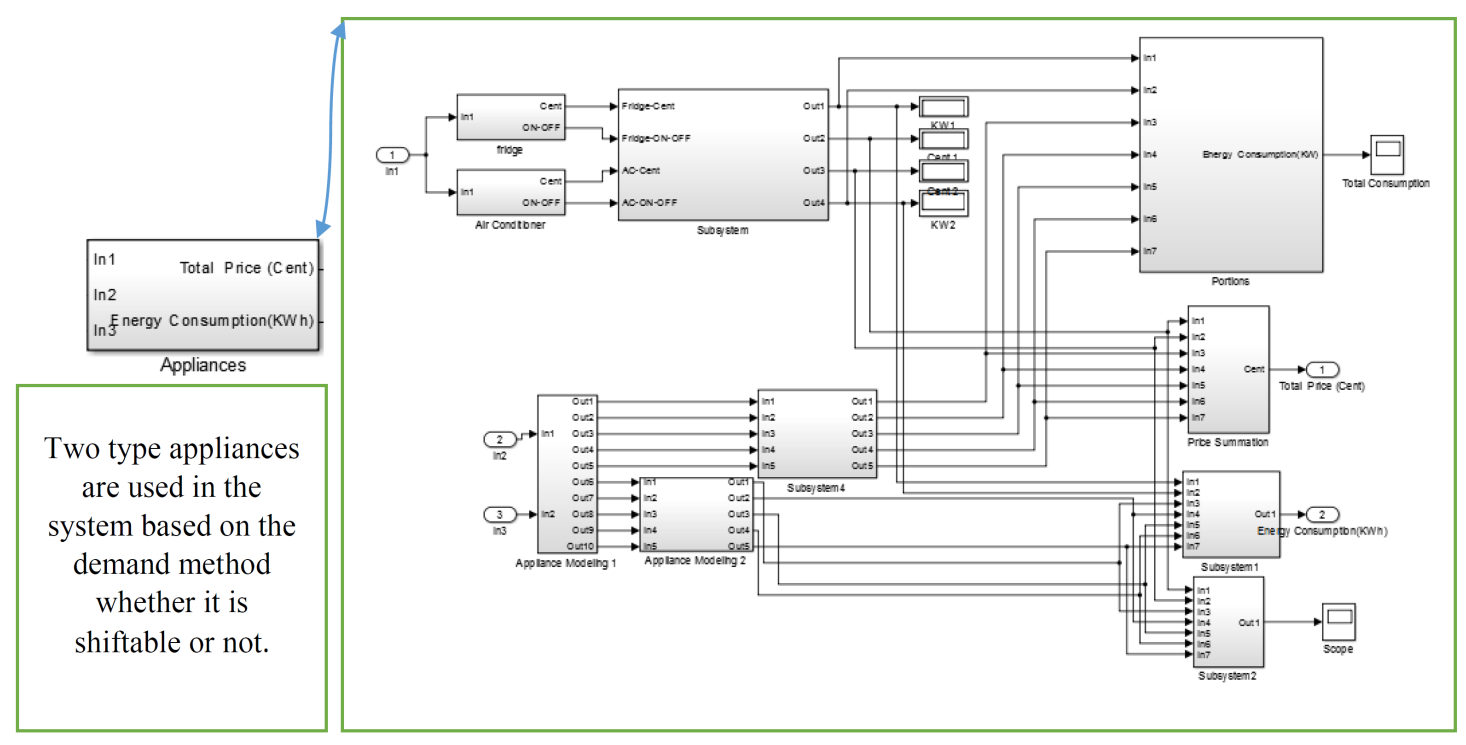

Figure 2.16: General View of Appliances

applied into the shiftable appliances, see Figure 2.16. Fridge's demand is an examples of a non-shiftable demand; therefore, it is modeled in a different way. The Bernoulli method used to model the fridge. In each 10 minutes cycle, the fridge turns $\mathrm{ON}$ and OFF based on the real time data, see Figure 2.17. To recover the systems model, we adjust some parameters for the non-shiftable demand. We applied this method to shifitable demand as well, see Figure 2.18. The Portion box multiplies some constant variables with shiftable demand. These values are changeable in different appliances, see Figure 2.19. The Price Summation box sums all prices based on the consumed energy from the users sides, see Figure 2.20. Appliance Model box multiplies the consumed energy by some fixed numbers to realize the total price paid by end users, see Figure 2.21. The Appliance Modeling 1 box sums all consumed energy by 


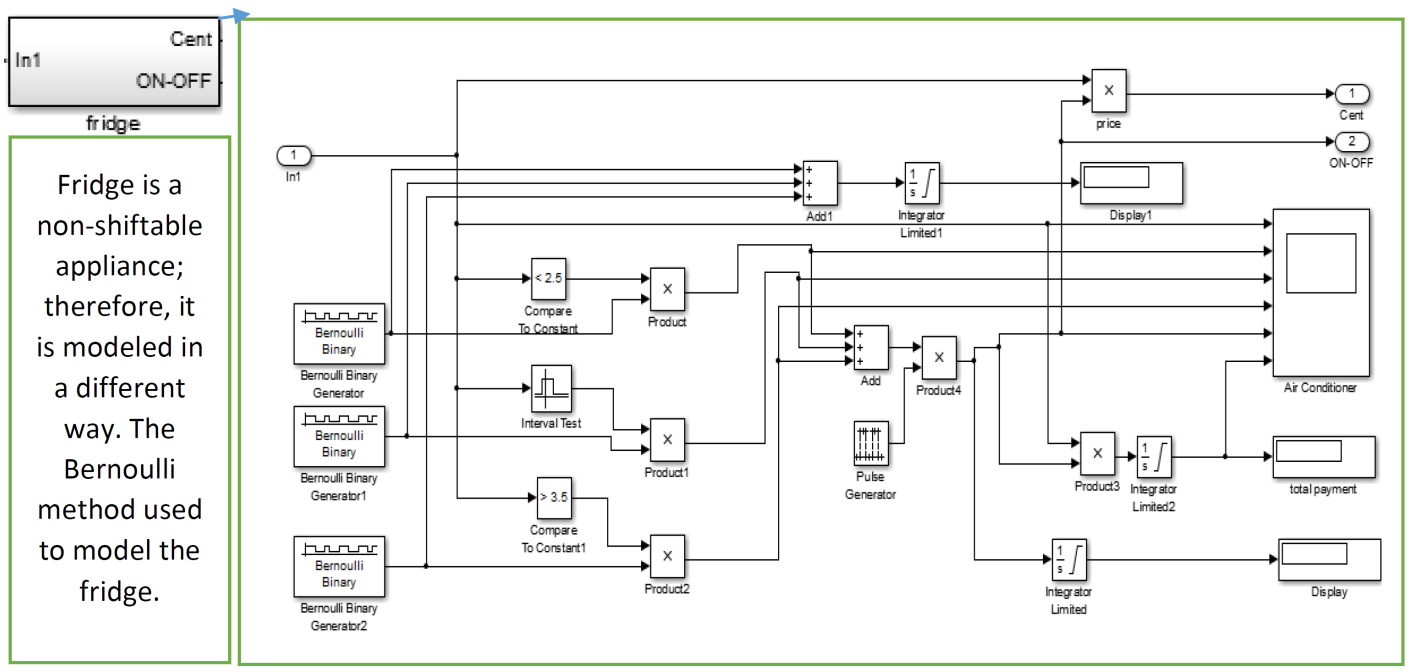

Figure 2.17: Fridge is an Example of non-Shiftable Demand

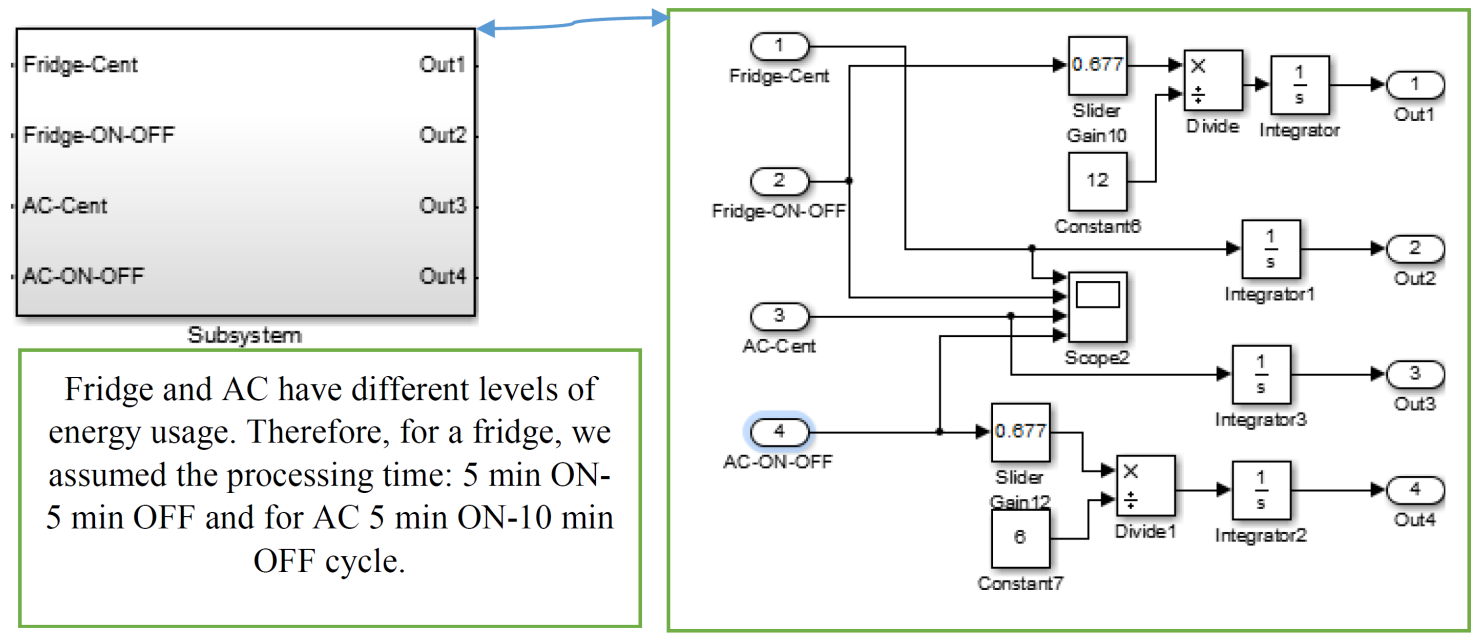

Figure 2.18: The Recover Parameters for non-Shiftable Demand 


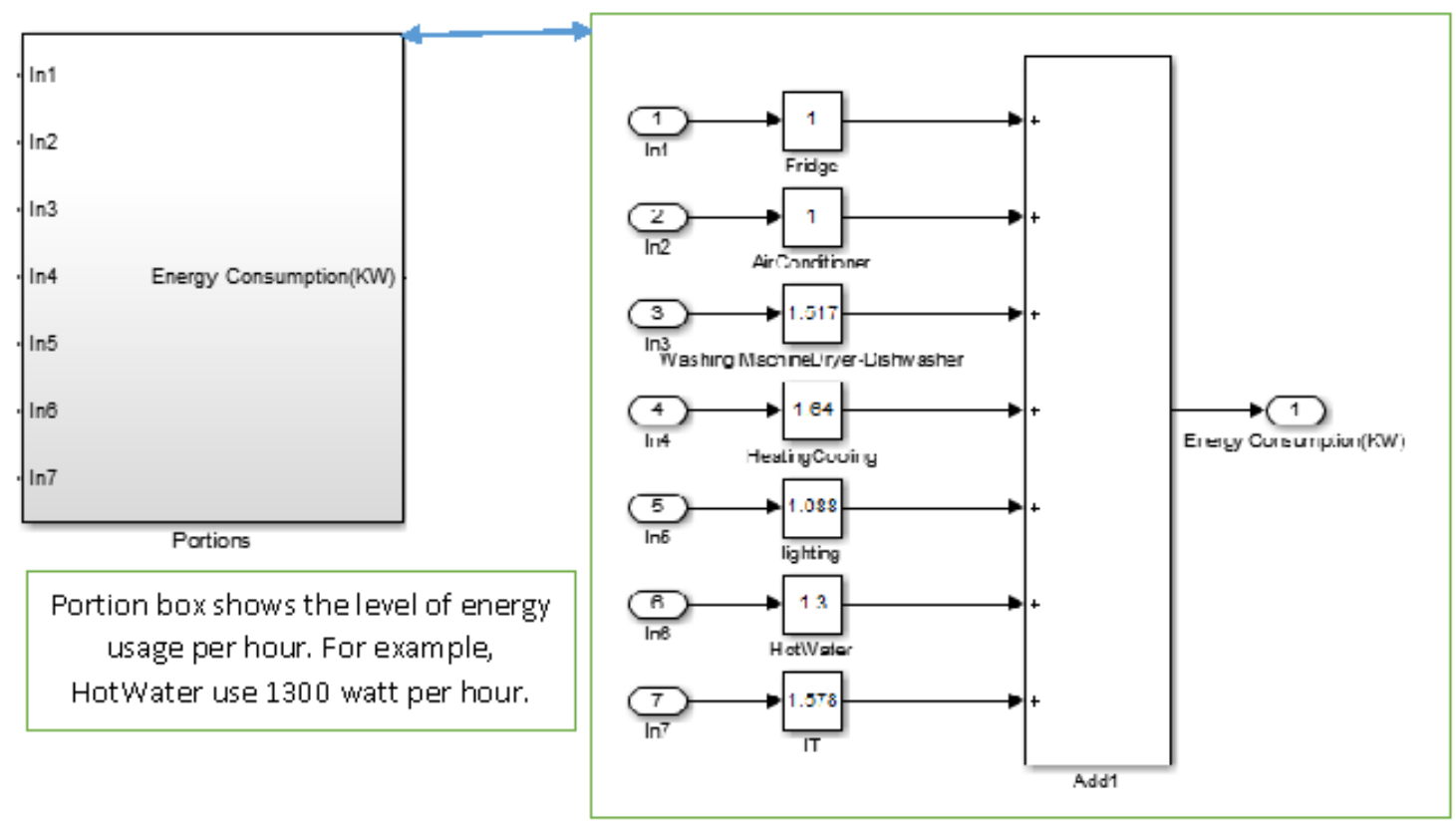

Figure 2.19: Constant Values for Appliance Models

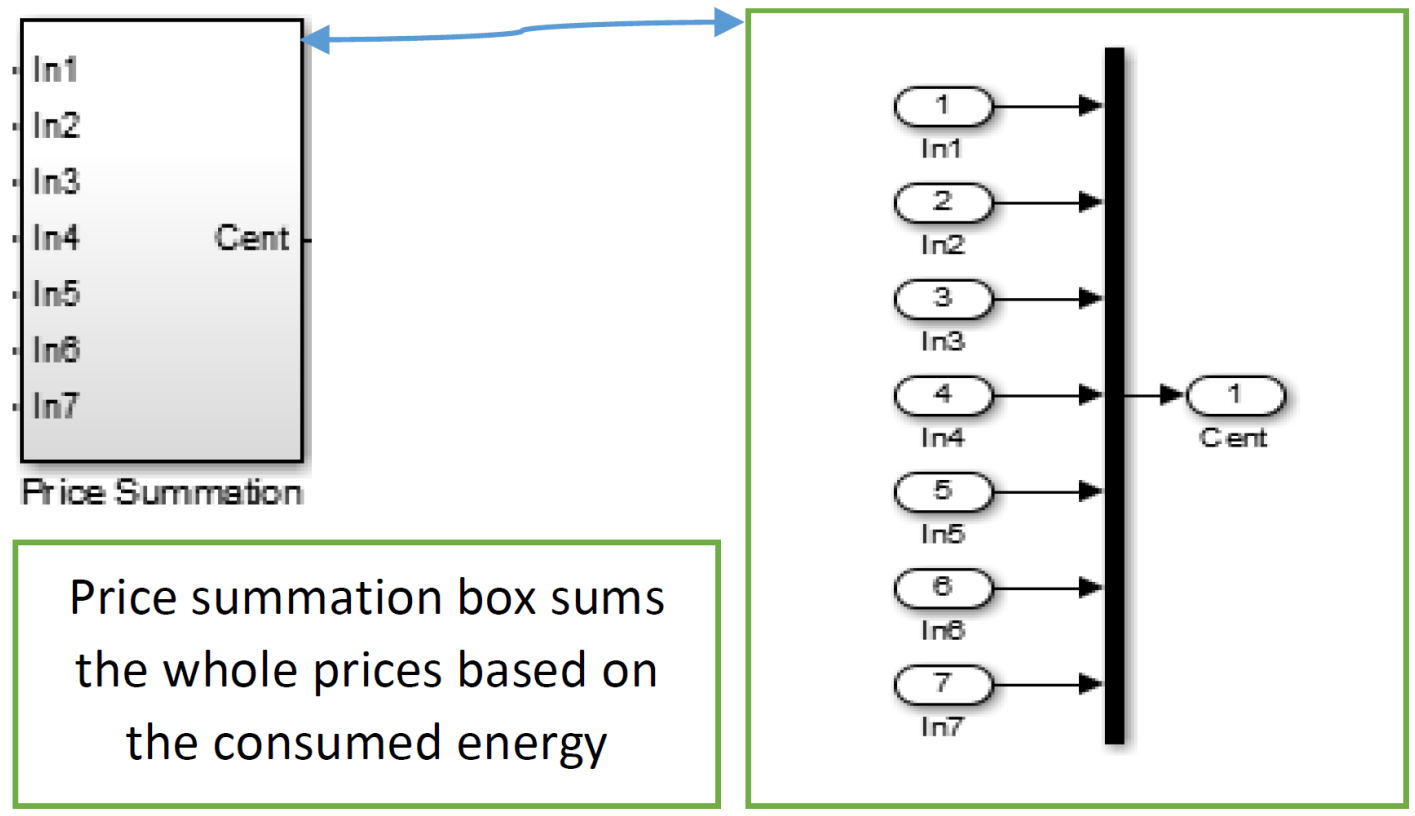

Figure 2.20: Prices are Calculated Based on the Consumed Energy 


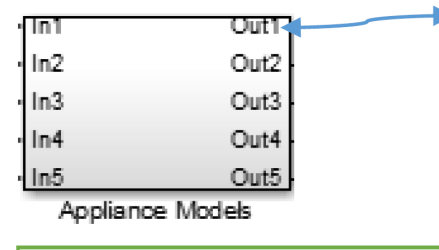

Appliance model box multiplies the consumed energy by the fixed number, to realize the total price paid by end users.
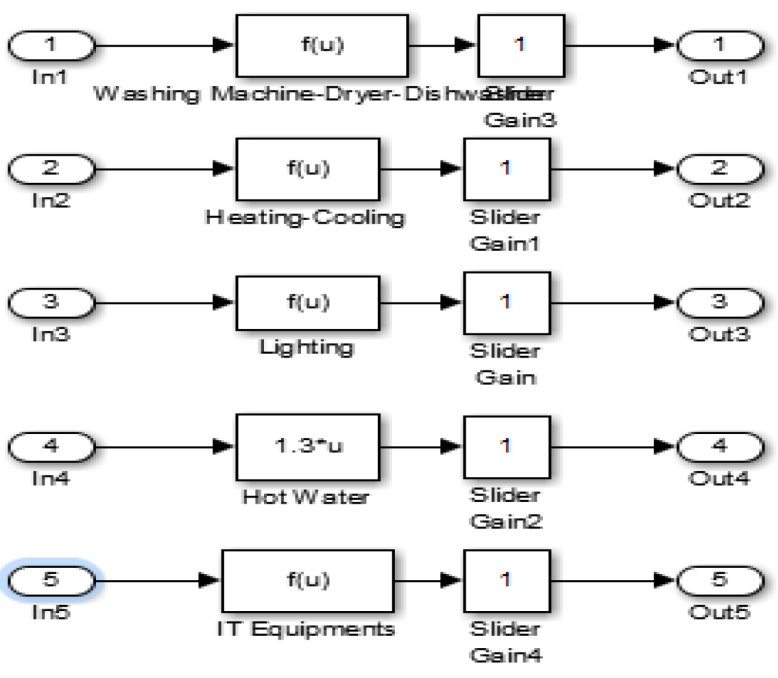

Figure 2.21: Calculating the Total Payment

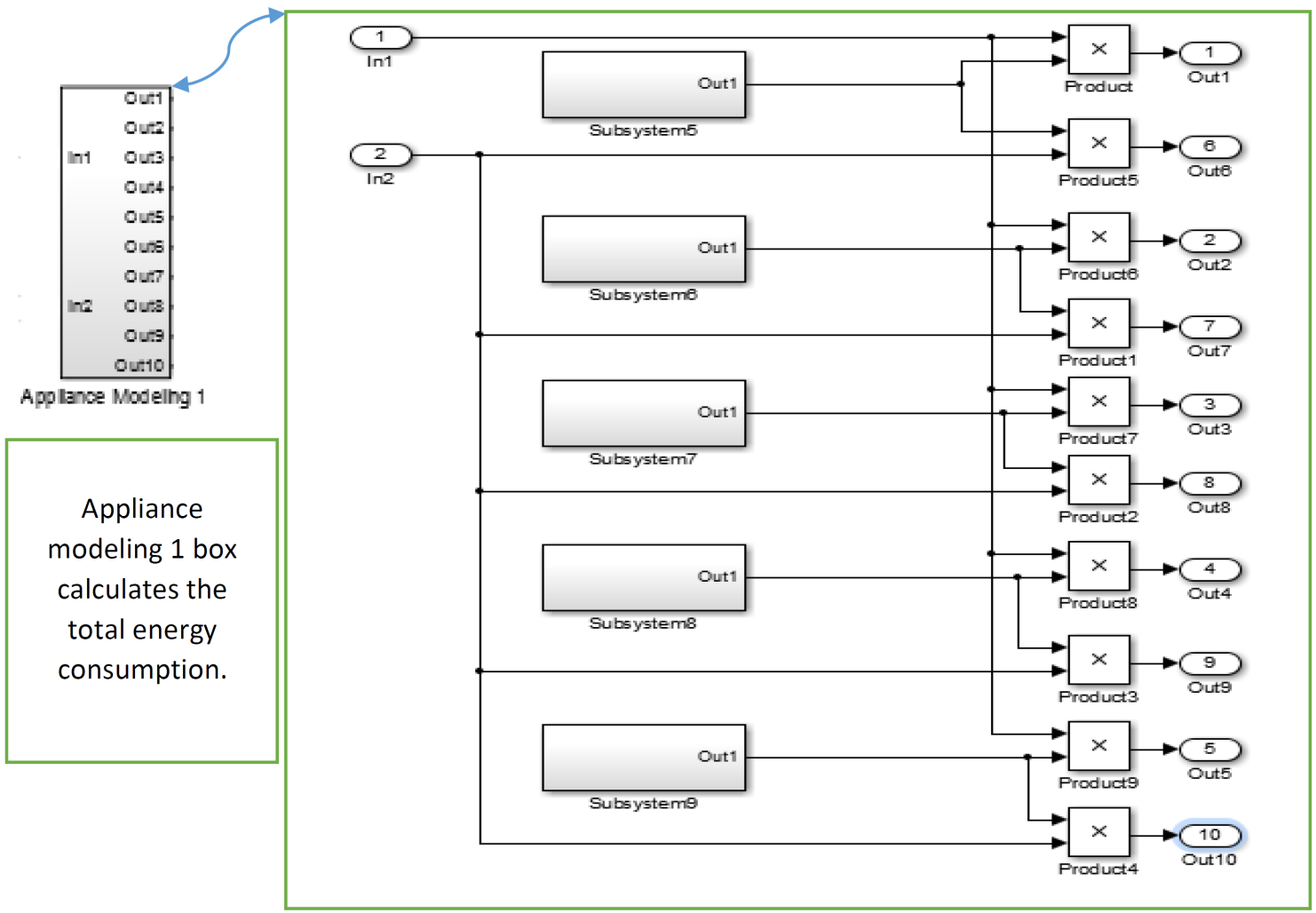

Figure 2.22: Calculating the Total Consumption 
appliances in the user side, see Figure 2.22.

\subsection{Independent Electricity System Operator (IESO)}

The Independent Electricity System Operator (IESO) works at the heart of Ontario's power system. It ensures whether or not there is enough power to meet the province's energy needs in real time. Moreover, IESO is planning and securing energy for the future. It does this by:

- Balancing the supply of and demand for electricity in Ontario and directing its flow across the province's transmission lines.

- Planning for the province' medium and long-term energy needs and securing clean sources of supply to meet those needs.

- Overseeing the electricity wholesale market where the price is set.

- Fostering the development of a conservation culture in the province through programs such as saveONenergy.

Ontario's IESO works to ensure sufficient electricity is available whenever and wherever it's needed. Ensuring there is enough energy to meet demand is an ongoing and highly complex process, requiring the close coordination of all parts of the system.

Every five minutes, the IESO forecasts electricity demand throughout the province and directs generators to provide the required amount of electricity to meet that demand. It can also reduce demand by calling on large volume users to cut back on 


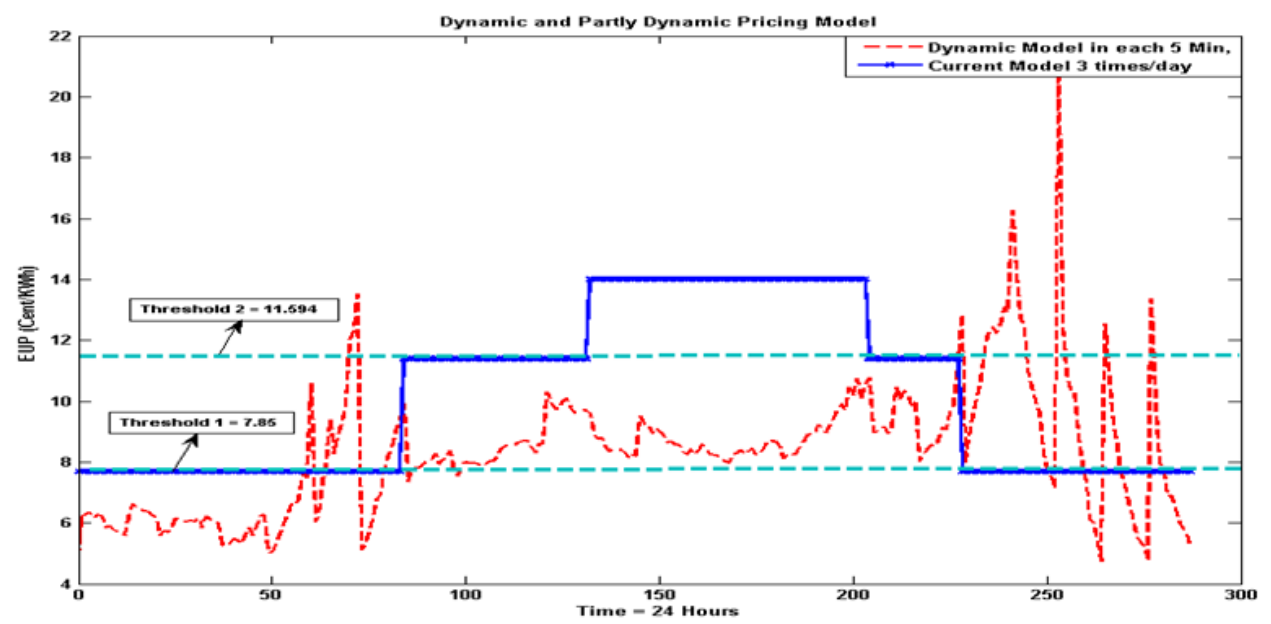

Figure 2.23: Dynamic, Current, and Fixed Pricing Models

\begin{tabular}{|c|c|c|c|c|c|}
\hline Green & Improvement & Yellow & Improvement & Red & Improvement \\
\hline 7.75 vs 7.85 & $1.2 \%$ & 8.84 vs 11.594 & $23 \%$ & 8.75 vs 11.594 & $>23 \%$ \\
\hline $7 \mathrm{pm}-7 \mathrm{am}$ & $5 \mathrm{pm}-7 \mathrm{pm}$ and $7 \mathrm{am}-11 \mathrm{am}$ & $11 \mathrm{am}-5 \mathrm{pm}$ & \\
\hline
\end{tabular}

Table 2.1: Improvements in Dynamic, Current,and Fixed Pricing Models (Cent)

consumption. This process of balancing supply and demand repeats itself 24 hours a day, seven days a week.

We used IESO databases as reference sources that enables 5 minute instant price to the control system. As can be seen from Figure 2.23, the current pricing model is divided into three sections. In other words, on a particular day end user price (EUP) is changing three times between $7.8,11.4$, and 14 cents per KWh. On the other hand, we have proved that the 5 minute dynamic model is not matched with the current pricing model that is still in use in Ontario. As can be seen, the total average price in the green section is high due to the fluctuated instant electricity price. On the other hand, the Table 2.1 indicates that the average fluctuated EUP in the yellow and red ranges in the dynamic model is less than the current pricing model. This means that users get benefits when they use their appliances in a fully dynamic model because 
the real time price (in 5 minute cycle) is less than the current price. Currently, users pay according to the current pricing model. On the contrary, when end users turn on their appliances in the yellow or red ranges based on the current pricing model, they have to pay higher than usual because real time pricing is less than the current pricing model.

\subsection{Summary of This Chapter}

Our goal is to encourage end users to be aware of the high electricity prices when it is higher than the regular constructed price. Since, end users avoid turning on their appliances at the high priced time, based on supply-demand relation, the peak price goes down and leads to a more smooth and efficient pricing range. In case of congestion in demand after a point, end users are supposed to apply their demand to block the intensity. 


\section{Chapter 3}

\section{Open-Loop Control Model}

Open-loop control model doesn't have any feedback to determine whether its output has achieved the desired goal of the input. An electric clothes dryer would be a good example of a device having an open-loop mechanism. Depending on the amount of clothes or how wet they are, a user would set the timer (controller) of dryer as 30 minutes. 30 minutes later the dryer would automatically stop, even if the clothes were still wet or damp, see Figure 3.1.

For an open-loop control (OLC) model, we have examined UIL under three types of pricing models (fixed, current, and dynamic) in terms of the number of users and appliances, see Figure 3.2.

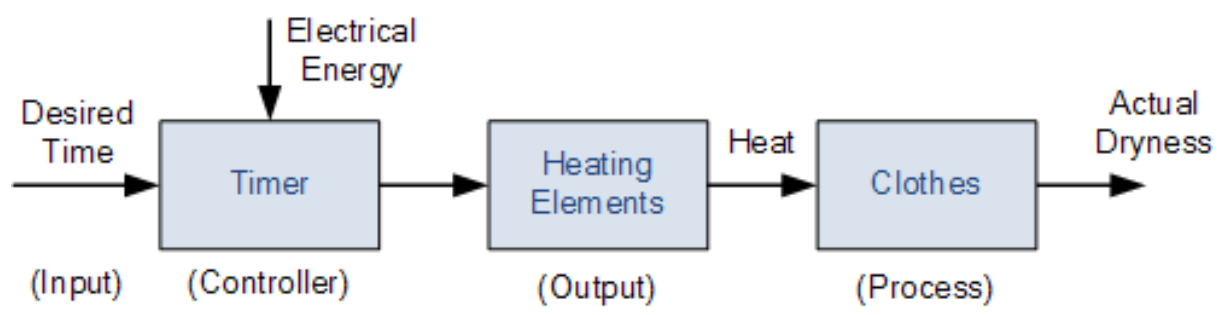

Figure 3.1: Example for Open-Loop Control Model 


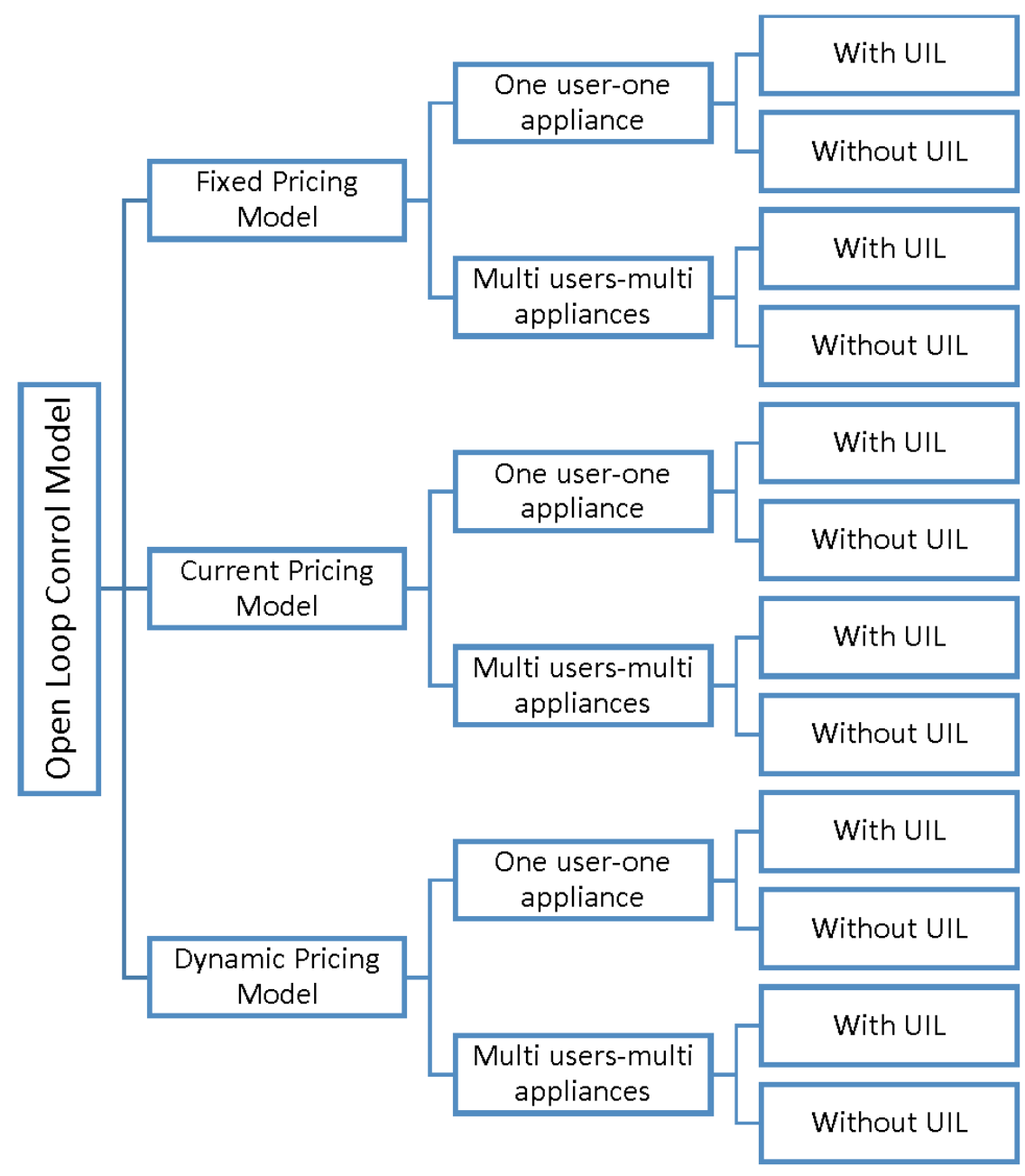

Figure 3.2: Open-Loop Control Model Road Map 


\subsection{Fixed Pricing Model}

In fixed pricing model, the EU price remains stable during the whole year. Therefore, users cannot obtain any benefit such as saving money. In other words, end users pay depending on their usage, see Figure 3.3. Therefore, there is a direct relationship between the total usage and the total payment as seen in the equation below:

$$
\begin{aligned}
& Y_{i}=\alpha+\beta_{i} * X_{i} \\
& i=1,2, \ldots, \\
& \alpha=\text { constant variable } \\
& \beta=\text { independent variable }
\end{aligned}
$$

In the above, $Y_{i}$ is the total price in which end users pay for a one time turning on their appliance; $X_{i}$ is the base price that is calculated from the supply-demand relation. Then, $\alpha$ is a constant variable extracted from the inflation rate, interest charges, distribution fees, smart meter charge, etc. Likewise, $\beta$ refers to how long the electricity has been used.

In the following subsections, I will explore fixed pricing model regarding the number of users and appliances.

\subsubsection{One-User One-Appliance Model}

Through this model, the demand for electricity is shifted in time domain because there is a positive relationship between demand and price. Since end user price remains stable during the whole year, there is no need for a threshold value for this model. Since we have only one single user and only one single appliance, the distribution of the demand changes based on user's need for energy. Hence, 


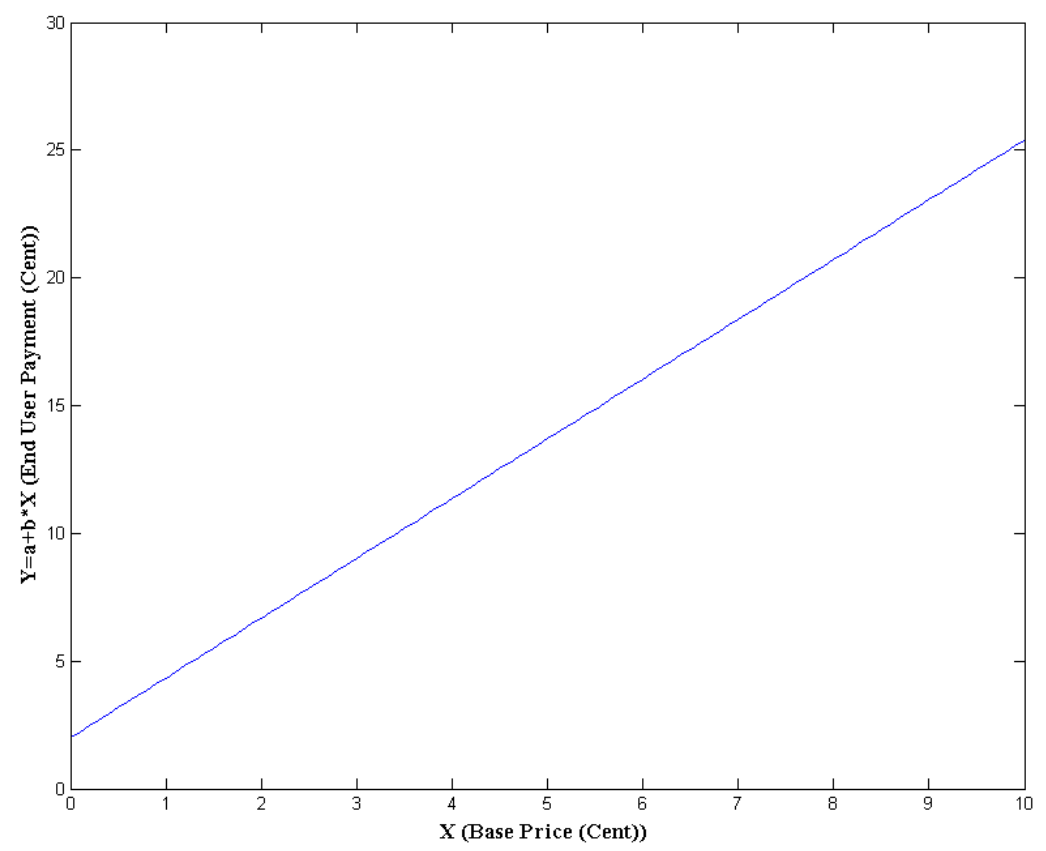

Figure 3.3: Linear Graph

$$
A=\alpha+\beta * B
$$

is a valid formulation for one-user one-appliance model. In the above, $A$ is the total payment in Cent that end user pays for one cycle of using his appliance, $B$ is the sum of the base price that the appliance is in $\mathrm{ON}$ mode and $\alpha$ is constant variable, $\beta$ is independent variable for tunning the base price with EU price.

\subsubsection{One-User Multi-Appliance Model}

For one-user multi-appliance model, it has similar properties with one-user oneappliance model due to constant price during the whole year. There is only one concern, which a user turns on his/her all appliances at the same time. Such causes high load which may partially damage his/her house system. 


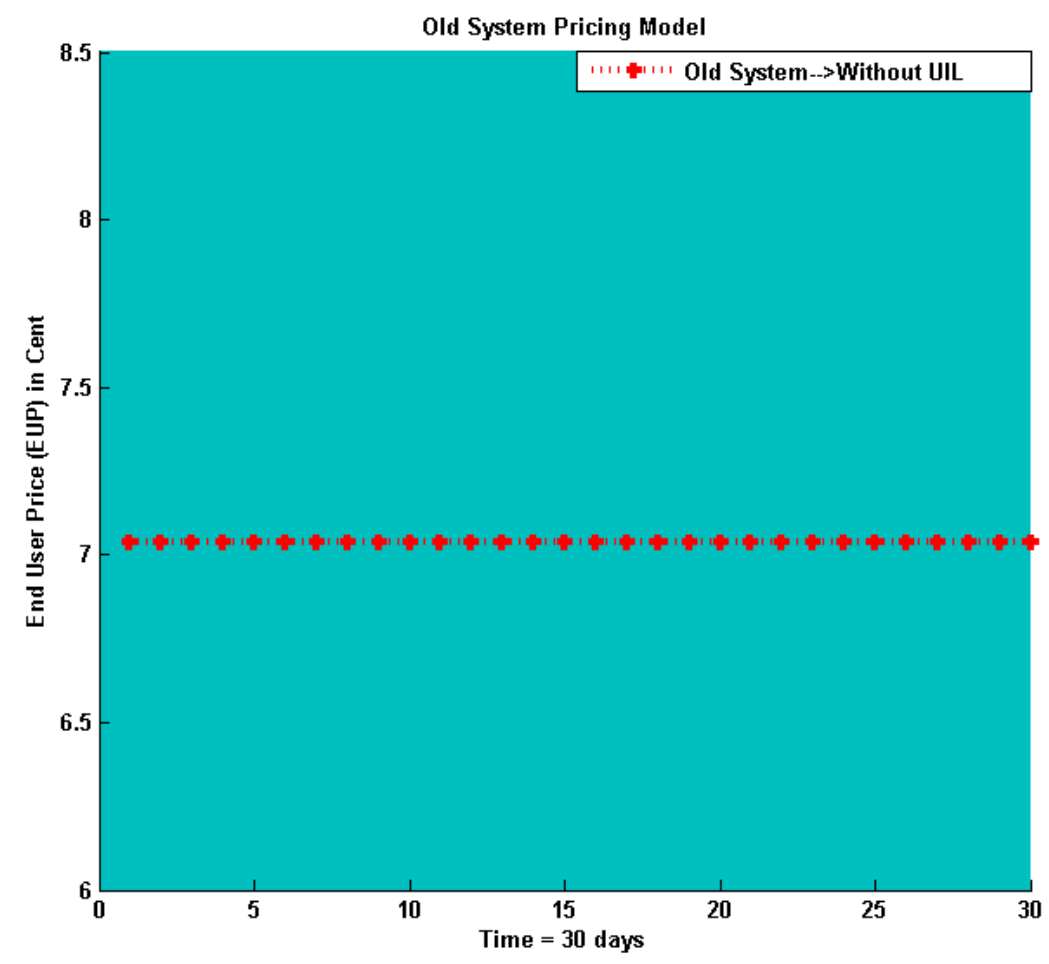

Figure 3.4: Fixed Pricing Model

\subsubsection{Multi-User Multi-Appliance Model}

Multi-user multi-appliance model is similar to the first and second models, but it is more complicated due to increase in the high number of consumers and appliances. Since the number of end users and appliances increases, the probability of power outage rises. Due to these reasons, the probability of corruption in the electricity grid increases. Another reason for the outage is that if smart communications are not installed in the fixed grid model, the supplier cannot realize the demand of end users. Therefore, the convergence of supply-demand cannot be estimated, see Figure 3.4.

To summarize, the fixed pricing model is examined in three cases. We can conclude that setting any threshold values is not required due to stable price during the billing 
period. Additionally, UIL method is not applicable because of undefined threshold values.

\subsection{Current Pricing Model}

In the current pricing model, three time scales (green, yellow, and red ranges) are used to settle the best convergence of supply and demand. When the difference between supply and demand is low, it means the base price goes down, until point $\alpha$. Likewise, if the difference between supply and demand is high, the base price goes up, to a high point. At this point, the supply side has to converge with demand; otherwise, neither the base price (end user price) goes down, nor the goal of energy saving is reached.

Unlike the fixed pricing model, current pricing model is analyzed with respect to only multi-user multi-appliance model because this pricing model is presently used for a large number of users and appliances.

\section{Defining Threshold Values}

Threshold values are calculated based on the probability of the three different pricing scales in which the base price and end user price are identified. We proposed that end users operate their appliances when the base price is under $\tau_{1}$. This means that during the green scale, which is already priced by the Energy Board of Ontario, users are supposed to be in this scale. This has some benefits to end users; for instance they have an opportunity to turn on their appliances in low price range, see Figure 3.5 .

A probability density function is most commonly associated with absolutely continuous univariate distributions. A random variable $\mathrm{X}$ has density $f_{X}$, where $f_{X}$ 

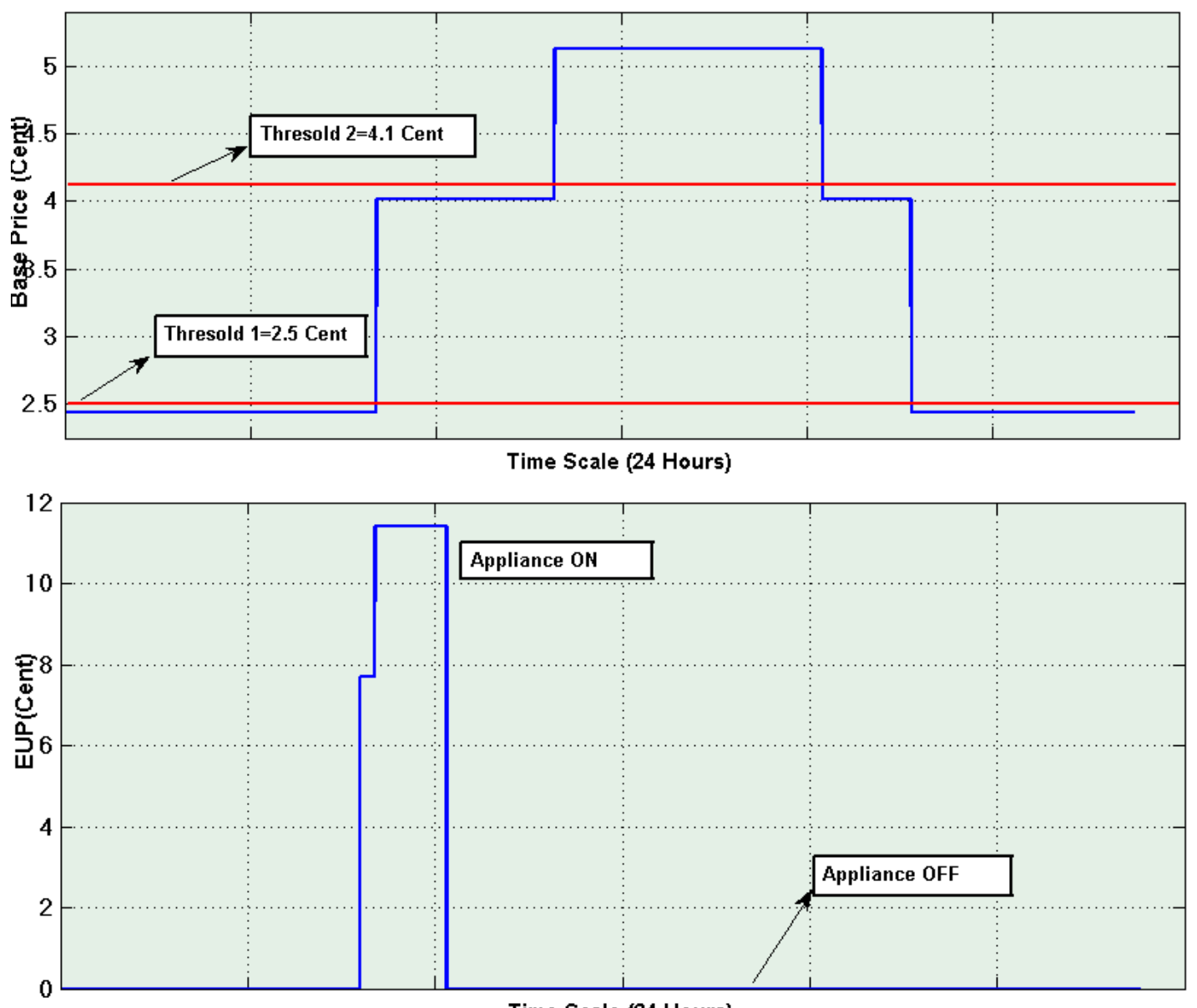

Time Scale (24 Hours)

Figure 3.5: Current Pricing Rate with Threshold Values 

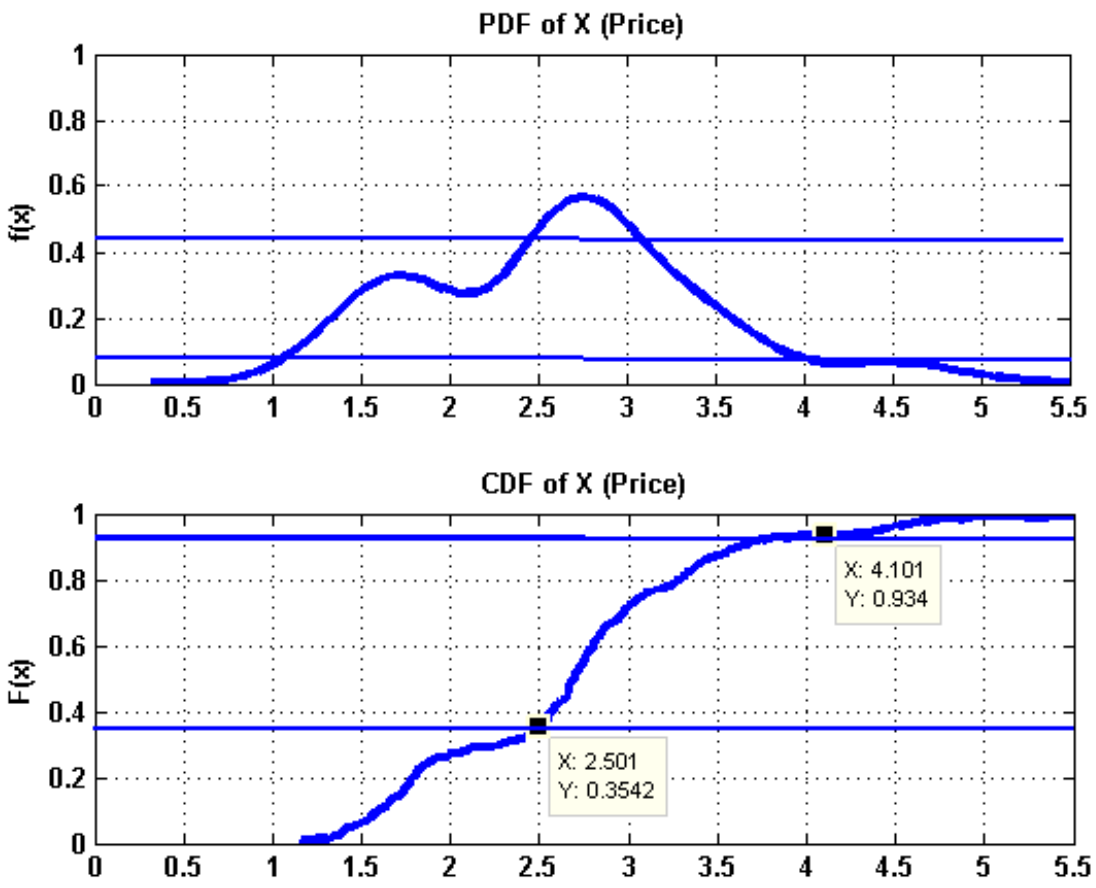

Histogram of $\mathrm{X}$

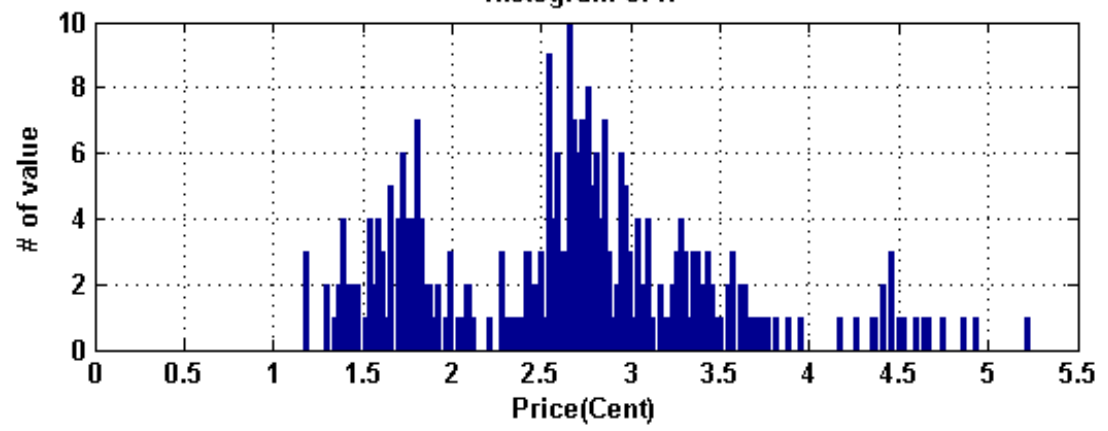

Figure 3.6: PDF, CDF and Histogram of X (Base Price) 
is a non-negative Lebesgue-integrable function, if

$P[a \leq X \leq b]=\int_{a}^{b} f_{X}(x) d x$

Hence, if $f_{X}$ is the cumulative distribution function of $x$, then

$F_{X}(x)=\int_{-\infty}^{x} f_{X}(u) d u$.

If $f_{X}$ is continuous at $\mathrm{x}$

$f_{X}(x) d x=\frac{d}{d x} F_{X}(x)$.

As can be seen from Figure 3.6, the PDF of base price is different from regular PDFs. This results from different price scales that change during the day. On the other hand, we understand that $36.4 \%$ of prices are in the green range while it is $57 \%$ in yellow and $6.6 \%$ in the red range. However, Hydro Ottawa offers that $50 \%$ of prices are in the green range while it is $25 \%$ in yellow and $25 \%$ in the red ranges. It can be clearly realized that the current pricing model that this company suggests does not indicate the real pricing scale. This is resulted from an unstable relation between the supply and demand curves. If this balance breaks down, the price goes up or down. Next,this problem is associated with instant changes in energy consumption and generation. We know that the alternative current (ac) energy cannot be stored. Energy consumption and generation has to be done at the same time. Energy demand changes very fast; therefore, the supply does not meet the demand. This is the last reason for breaking down the supply - demand balance. If suppliers are not ready for instant change on the demand side, basically, the power 
systems can be damaged (costs billions of dollars) due to short cuts or other factors caused from this short cuts.

To prevent these problems, we have to define two different threshold values based on the CDF and PDF of the instant base prices. These threshold values help us to limit the price range regarding to its value, for instance,

Set $\tau_{1}=2.5$, Cents

Set $\tau_{2}=4.1$, Cents

as shown in Figure 3.6

In the below we give a basic algorithm of making the best decision about time to turn on the appliance:

\section{Algorithm 1}

if

$$
\begin{aligned}
& \text { BasePrice } \leq \tau_{1} \\
& \operatorname{Pr}_{O N}=1.0, \operatorname{Pr}_{O F F}=0.0
\end{aligned}
$$

else if

$$
\begin{aligned}
& \tau_{1} \leq \text { Base Price } \leq \tau_{2} \\
& P r_{O N}=0.5, P r_{O F F}=0.5
\end{aligned}
$$

else

$$
\begin{aligned}
& \text { BasePrice }>\tau_{2} \\
& P r_{O N}=0.0, P r_{O F F}=1.0
\end{aligned}
$$

end

Similarly, we can emerge end user price (EUP) based on these threshold values shown in the below. Our threshold estimation is as follows: 
$E U P=2+2.34 *$ BasePrice

for $\tau_{1}$

$E U P_{1}=7.8$ Cents

for $\tau_{2}$

$E U P_{2}=11.6$ Cents.

In the above, $E U P_{1}$ and $E U P_{2}$ define end user payment limits and range prices respectively in three different colours (green, yellow, and red). Similar to Algorithm 1, we can define the Algorithm 2 that is already identified by HydroOttawa. Based on the Algorithm 2, the $E U P_{1}$ shows the bound of green and yellow ranges while $E U P_{2}$ shows the bound between yellow and red ranges. In the current pricing model, $E U P_{C 1}$ is equal to 7.7 Cents and $E U P_{C 2}$ is equal to 11.4 Cents. It can be seen that our estimation matches the current pricing model. In other words, these threshold value estimations can be applied to dynamic pricing model.

\section{Algorithm 2}

if

$$
\begin{aligned}
& \text { BasePrice } \leq E U P_{C 1} \\
& \quad \operatorname{Pr}_{O N}=1.0, \operatorname{Pr}_{O F F}=0.0
\end{aligned}
$$

else if

$$
\begin{aligned}
& E U P_{C 1}<\text { BasePrice } \leq E U P_{C 2} \\
& \operatorname{Pr}_{O N}=0.5, \operatorname{Pr}_{O F F}=0.5
\end{aligned}
$$

else

$$
\begin{aligned}
& \text { BasePrice }>E U P_{C 2} \\
& \operatorname{Pr}_{O N}=0.0, \operatorname{Pr}_{O F F}=1.0
\end{aligned}
$$

end 


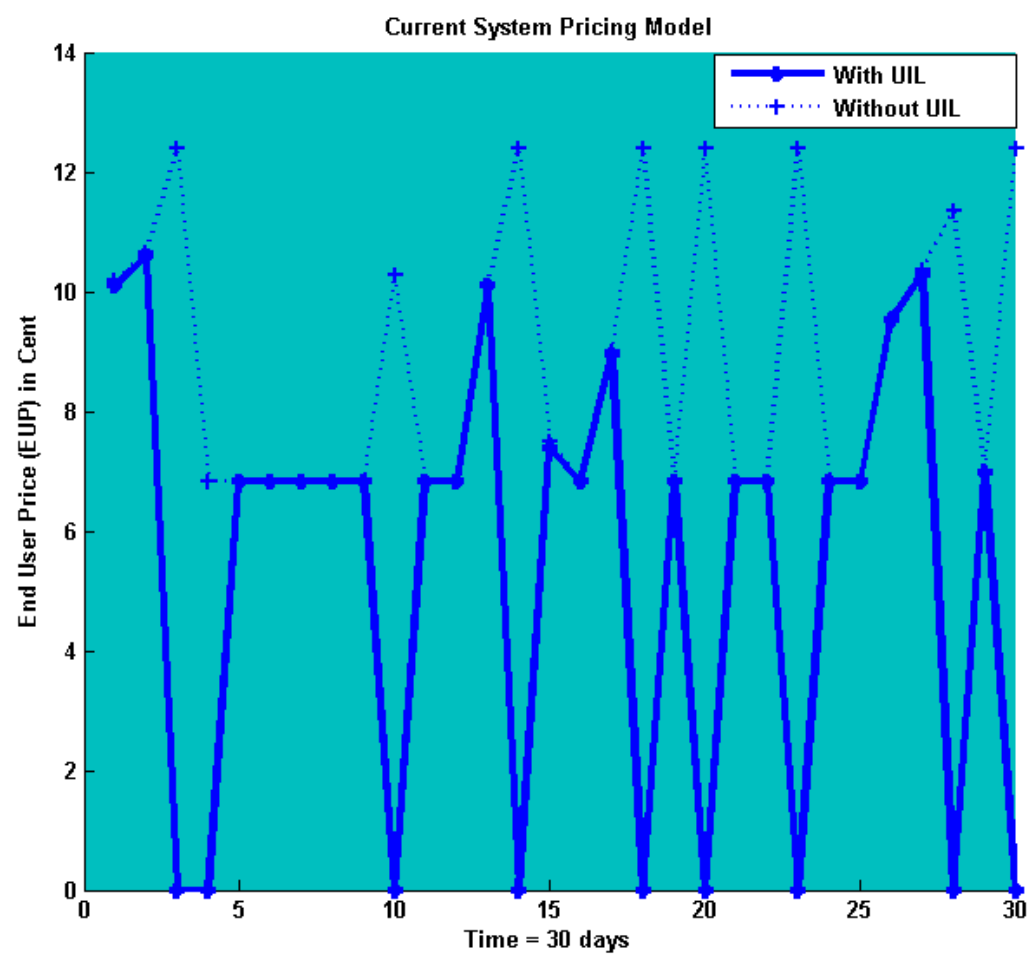

Figure 3.7: One-User One-Appliance Model

The simulation of the current model for a different number of users and appliances is shown in Figure 3.7.

When we use the UIL method in one-user one-appliance model, users reduce total price for $10 \%$ by comparing the system without using UIL method. Similarly, for multi-users multi-appliance, users still save money, around 5\%. When the number of users and appliances increase, using UIL partly lose its effectiveness. Since end users turn on their appliances during low price time, they save money. Moreover system gives information about the instant prices and avoid users be in high price time. When the price is high users are supposed to adjust their demand to next time. As can be seen from Figure 3.8, the appliance are not turned on in the red range where is $\tau_{1}=7.85$ (Cents/KWh) for green range. On the other hand, when user doesn't care 


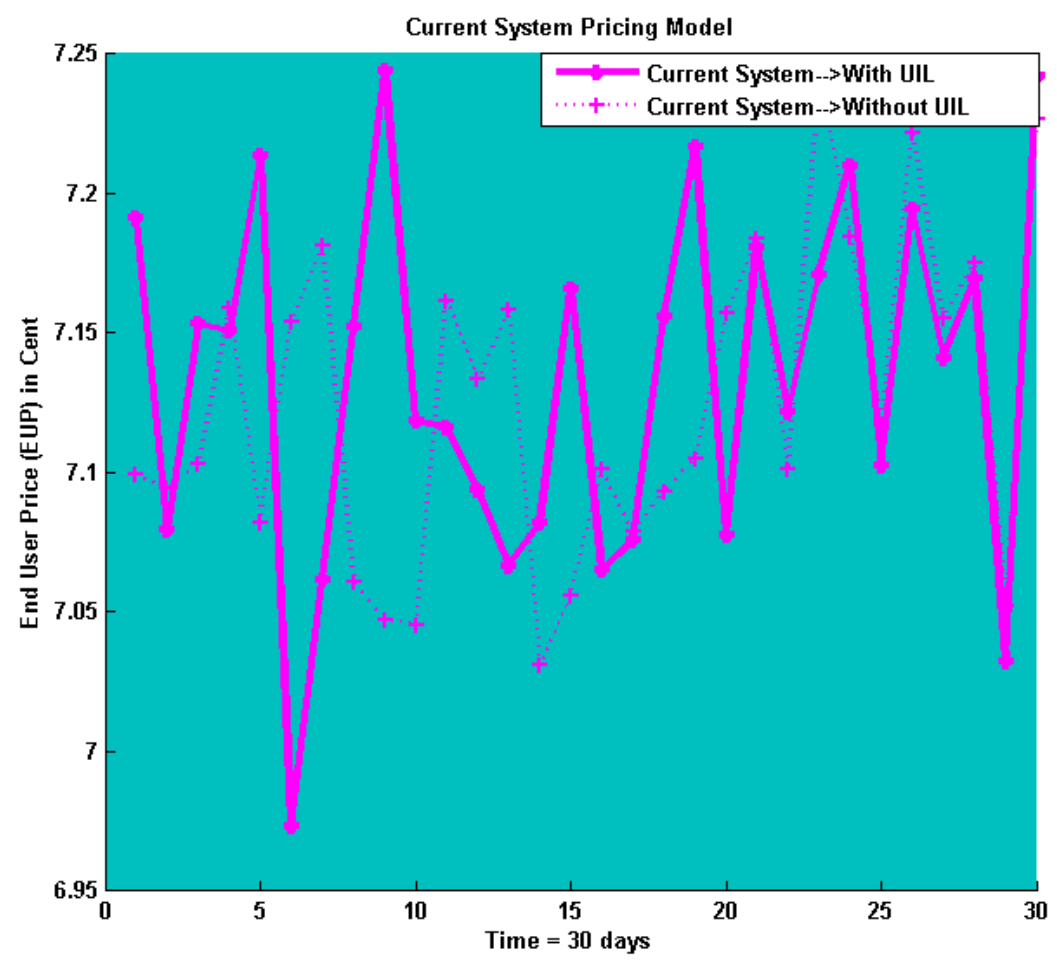

Figure 3.8: Multi-User Multi-Appliances Model 
the usage, the base price may be higher than $\tau_{2}=11.4$ (Cents/KWh) which is the second limit yellow range.

To sum up,the current pricing model does not meet the real criteria, because the base price is calculated instantly from the relation between supply and demand. Instead of three times scaling, we offer a fully dynamic pricing model that meet our criteria and works better than the current pricing model with the UIL method. When we use UIL method in the current pricing model, users save money around $7.5 \%$ in each billing period.

\subsection{Dynamic Pricing Model}

User in the loop (UIL) is the idea that end users are supposed to delay their usage to a later time. By doing this, end user stays away from being in high price time. The goal of UIL is to encourage end users to make an efficient decision in terms of lower prices. For the open-loop system, end user is supposed to make a decision either to turn on his appliance or not. However, this decision does not impact the input variables. This means that, user makes a decision only of a suitable time to turn on his smart appliances without any feedback to the supplier. In other words, the open-loop model does not have any feedback; therefore, the impact of end user on saving money is limited.

\subsubsection{One-User One-Appliance Model}

This model is the basic version of the whole system. The optimal time to turn on the appliance is the time when the cost of the electricity is low. End users can follow the instant prices on their smart appliances or cellphones in a 5 minutes cycle. On the contrary, for the open-loop model, we don't need feedback to the supplier; we only 


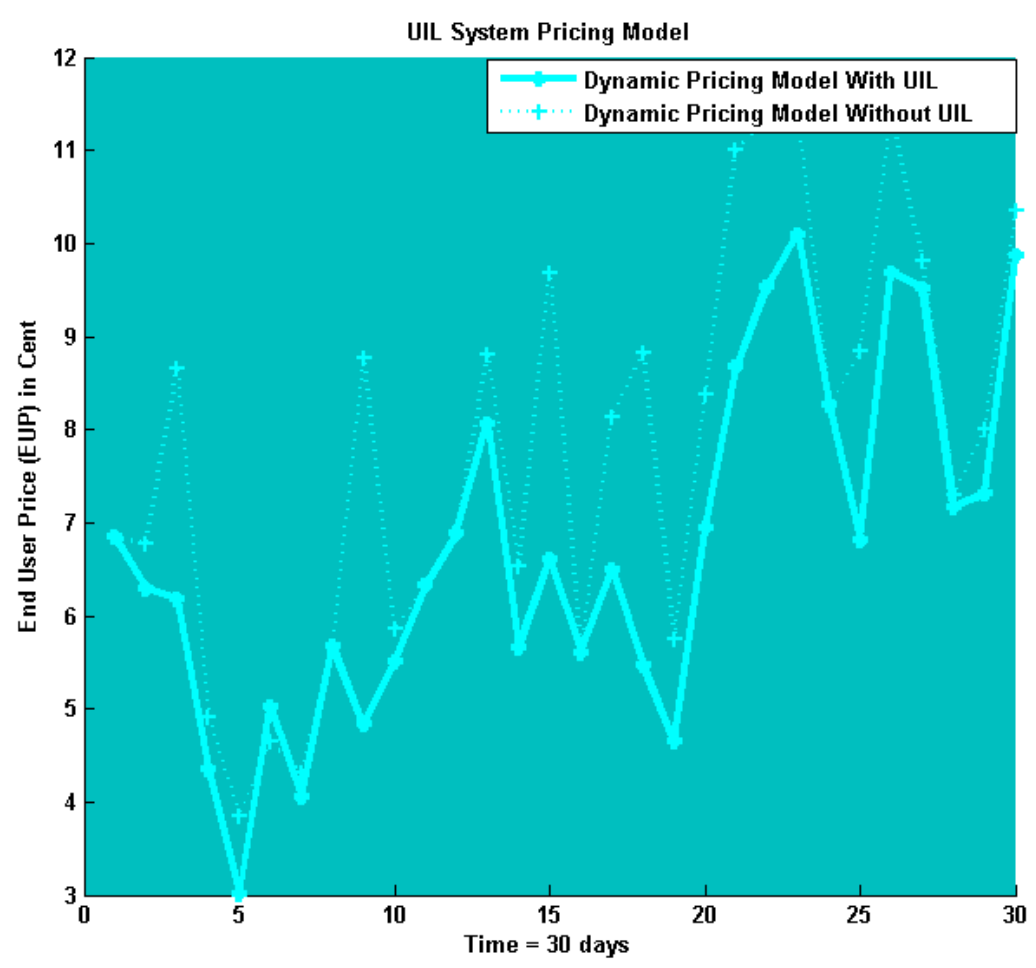

Figure 3.9: One-User One-Appliance Model

need backward information from the supplier to end users to make a decision. The threshold limits can be used in this model efficiently. Here is the comparison between UIL and no UIL model. It can clearly be seen in Figure 3.9 that if we have a limited number of users and appliances the system is more money efficient if UIL is used. The money saving in this model is more than $20 \%$ per KWh.

\subsubsection{One-User Multi-Appliance Model}

The one-user multi-appliance model is similar to the one-user one-appliance model, but it is slightly different. First, it is easier to control one appliance model than multi appliance. All appliances have to interact with each other to avoid being in the high 
cost range. To put it in other words, two appliances will not work at the same time. The only concern is if the number of appliances in ON mode is high, it can damage the house grid system.

\subsubsection{Multi-User Multi-Appliance Model}

Here is the complicated version of the whole model. If the system has many users, it is going to be more complex because all users have to interact with each other to prevent high load during the low cost time. Since the system is open-loop, it is impossible to provide interconnection among the all users around the city to specify the best time to turn on their appliances one by one. Here is the comparison of multi-users multi-appliance model, see Figure 3.10. Since the number of users and appliances is high we are not satisfied by using UIL method.

\subsection{Summary of This Chapter}

We built an open-loop control model. In this model, we analyzed UIL method under 3 different pricing models in terms of the number of users.

As a result, we understand that the UIL method enables efficient results for a small number of users. 


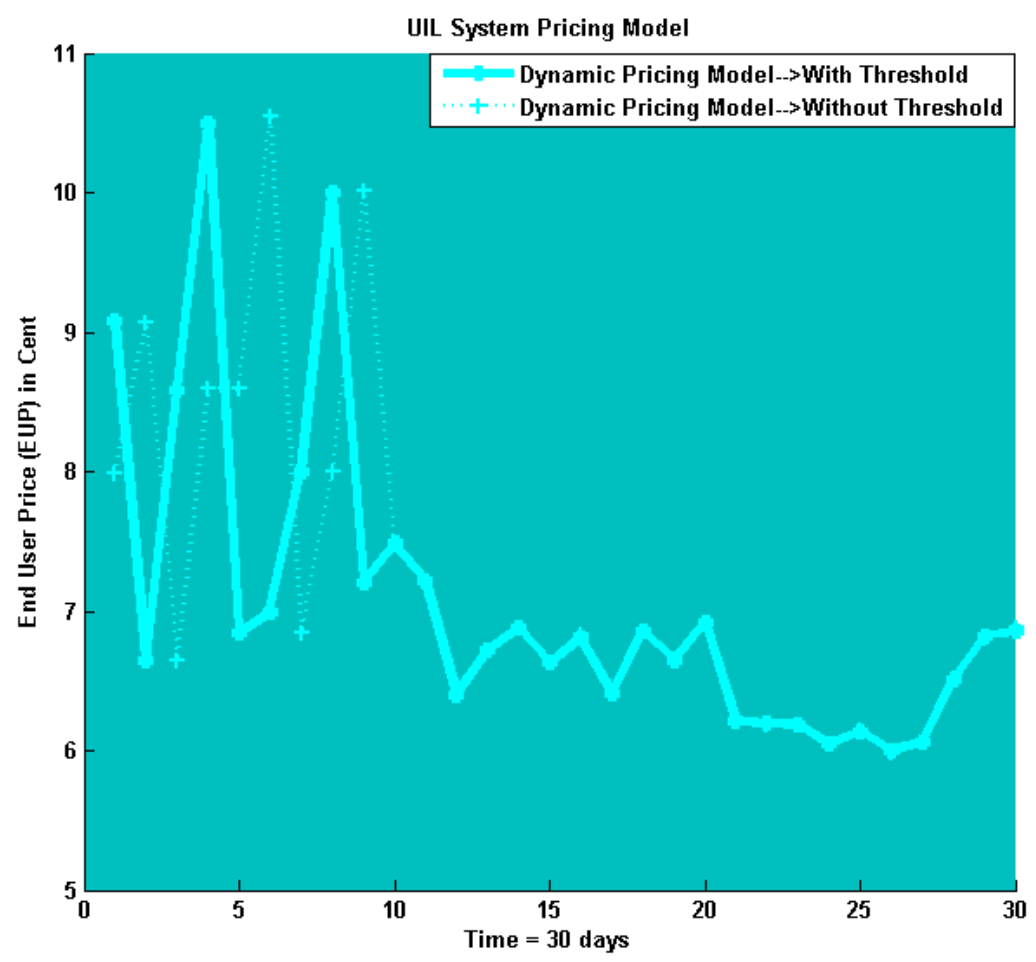

Figure 3.10: Multi-User Multi-Appliance Model 


\section{Chapter 4}

\section{Closed-Loop Control Model}

A closed-loop control system is known as a feedback control system. Unlike the open-loop control model, the closed-loop control model employs one or more feedback mechanisms. The reference to feedback simply means that some portion of the output is returned back to the input in order to form part of the systems excitation.

Closed-loop systems are designed to achieve and maintain the desired output condition by comparing reference input with the real-time output. This comparison is made through generating an error signal which indicates the difference between the output and the reference input.

Closed-loop systems can be integrated into home appliances such as an electric clothes dryer. Suppose that we have used a sensor or transducer input device) to continually monitor the dryness of clothes. This sensor would monitor the actual dryness of the clothes and compare it with (or subtract it from) the input reference. The error signal (error $=$ required dryness actual dryness) is amplified by the controller, and the controller output makes the necessary correction to the heating system to reduce any error. For example, if the clothes are too wet the controller may increase the temperature or drying time. Likewise, if the clothes are about to dry, either the temperature can be reduced or the dryer maybe stopped the process 


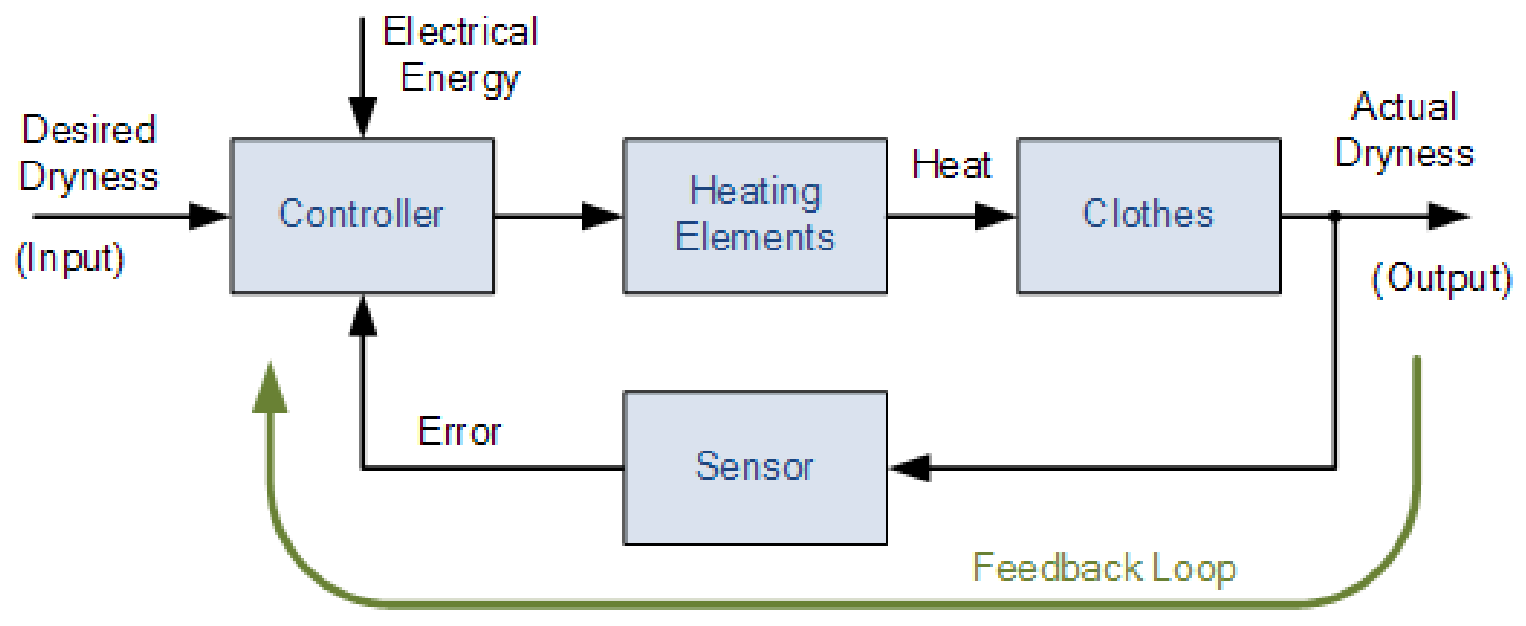

Figure 4.1: Closed-Loop Models

to prevent overheating the clothes, etc. See Figure 4.1.

The closed-loop configuration is characterized by the feedback signal derived from the sensor in clothes drying system. The magnitude and polarity of the resulting error signal would be directly related to the difference between the referent dryness and real-time dryness of the clothes.

When the deviation in temperature is detected by the feedback sensor and the controller self-corrects the error to maintain a constant temperature within the limits of the pre-set value, the dryer's door is opened or heat is reduced. Or, possibly, stops the process and activates an alarm to inform the operator.

As we can see, in a closed-loop control system the error signal is fed to the controller so as to reduce the systems error and bring the output of the system back to a desired value. Obviously, when the error is zero, the clothes are dry. Employing user in the loop method in closed-loop model results in super-Poisson distribution rather than uniform distribution. Therefore, relatively lower prices can be identified to meet end users' demand. In the UIL model, the controller is the proportional-integralderivative controller (PID controller), which controls the feedback loop mechanism 


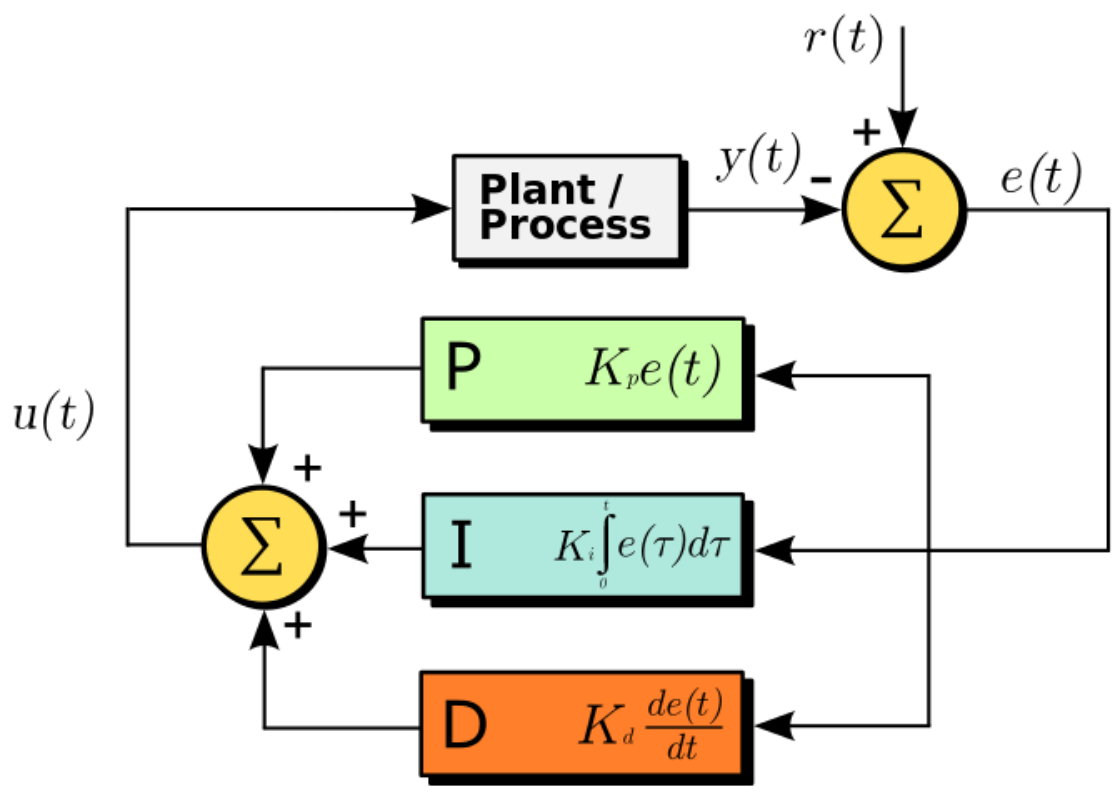

Figure 4.2: PID Controller

(controller), and it is widely used in industrial control systems. A PID controller calculates the error value by subtracting a measured process variable from a desired set-point. The controller attempts to minimize the error by adjusting the process through use of a manipulated variable, see Figure 4.2.

The equation below reveals how the error is computed and how the desired value is reached:

$u(t)=M V(t)=K_{p} e(t)+K_{i} \int_{0}^{t} e(\tau) d_{\tau}+K_{d} \frac{d}{d t} e(t)$

$K_{p}$ : Proportional gain, a tuning parameter

$K_{i}$ : Integral gain, a tuning parameter

$K_{d}$ : Derivative gain, a tuning parameter

$e$ : Error=SP-PV

$t$ : Time or instantaneous time (the present)

$\tau$ : Variable integration; takes on values from time 0 to the present $t$ 


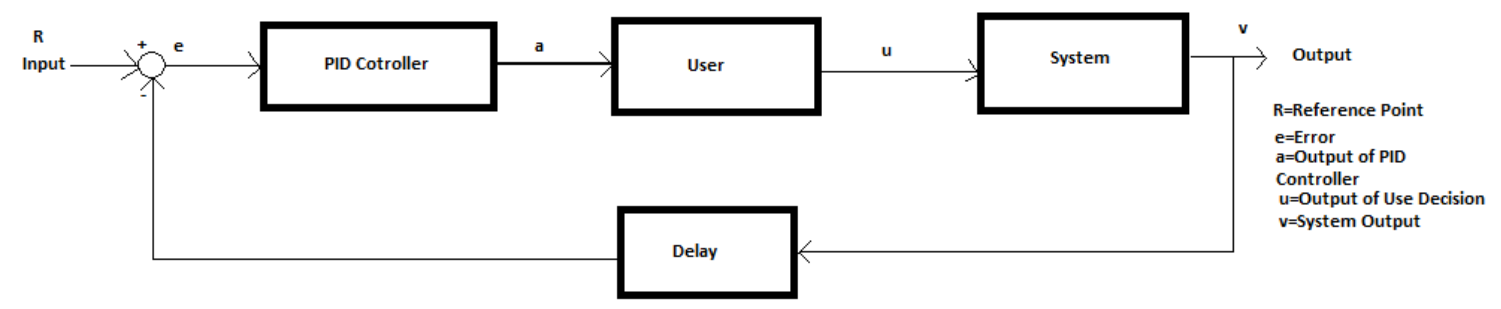

Figure 4.3: Closed-Loop Control Model

User box as demonstrated in Figure 4.3 is used for making decisions based on the output of the PID controller. The output of the PID is a price array that shows the lower prices that are under the $\tau_{2}$. This means that users see only the base price when it is under $\tau_{2}$; high prices are rejected. Users make decisions after they receive feedback for the low price rate. Afterwards, users send ON signal to the system. Based on the users' decisions, the system (smart appliances, smart vehicles, etc.) starts to progress. At the end of the progress, we see the total usage and payment at the output. Then, the energy is converted to end user price. The division of total usage by total payment (cent) gives us the EUP per KWh. Then, to find the base price, we do a reverse calculation of BasePrice $=(E U P-2) / 2.34$. Finally, we subtract this value from the input value to apply the PID model to the systems.

\section{Incentives Based on the End Users' Behaviors:}

End users' decisions are crucial in changing the mode of the appliances (ON or OFF). Therefore, they should be encouraged to delay their demand to suitable time. Dr. Schoenen et al did a survey that examines end users' tendency of delaying their demand at a latter of time when any incentives are provided. They found that when satisfactory incentives are given to end users such as a decrease in their monthly electricity bill or receiving bonus points, they tent to delay their demand at a latter 


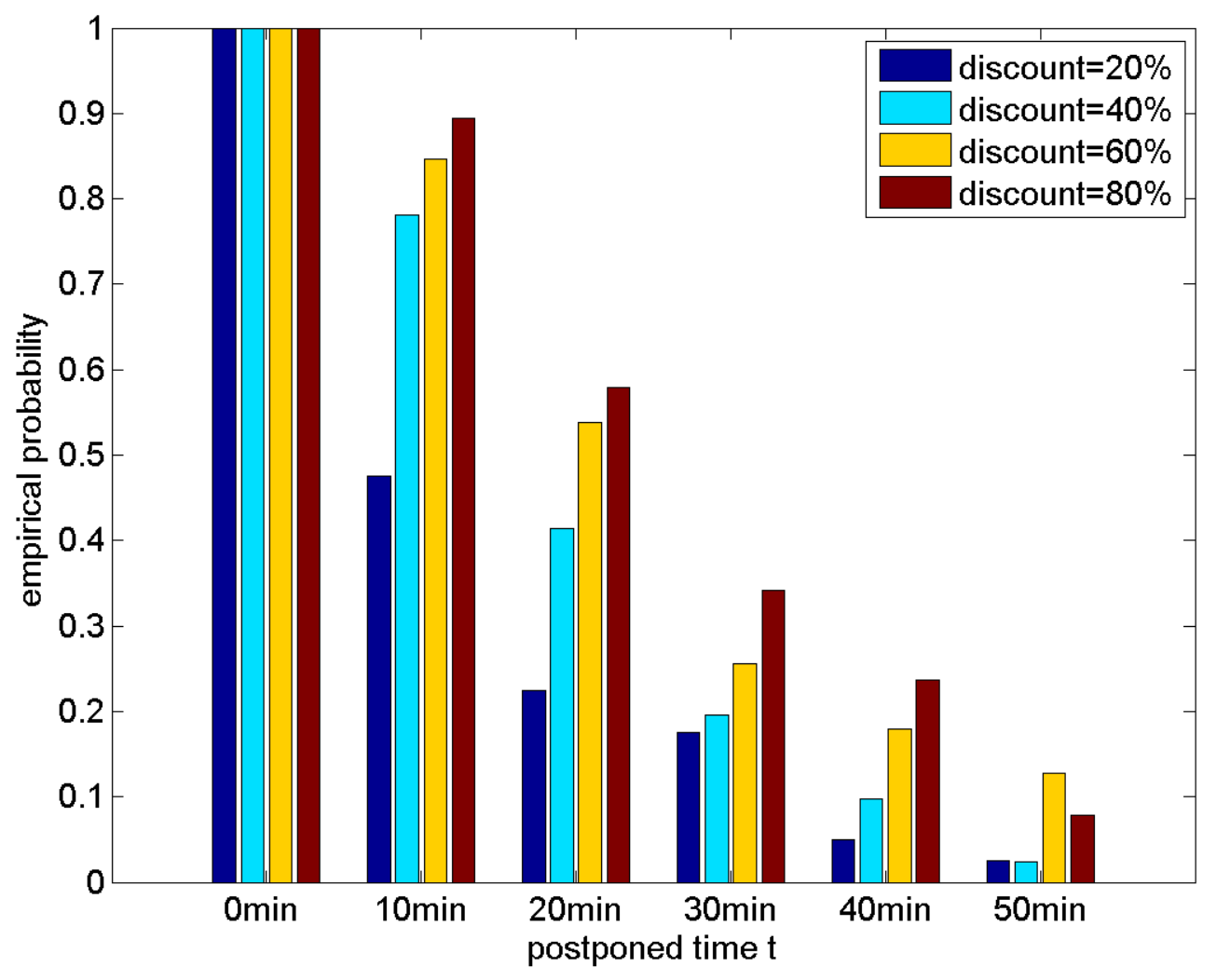

Figure 4.4: Temporal Probability with Postponed Time [12]. 


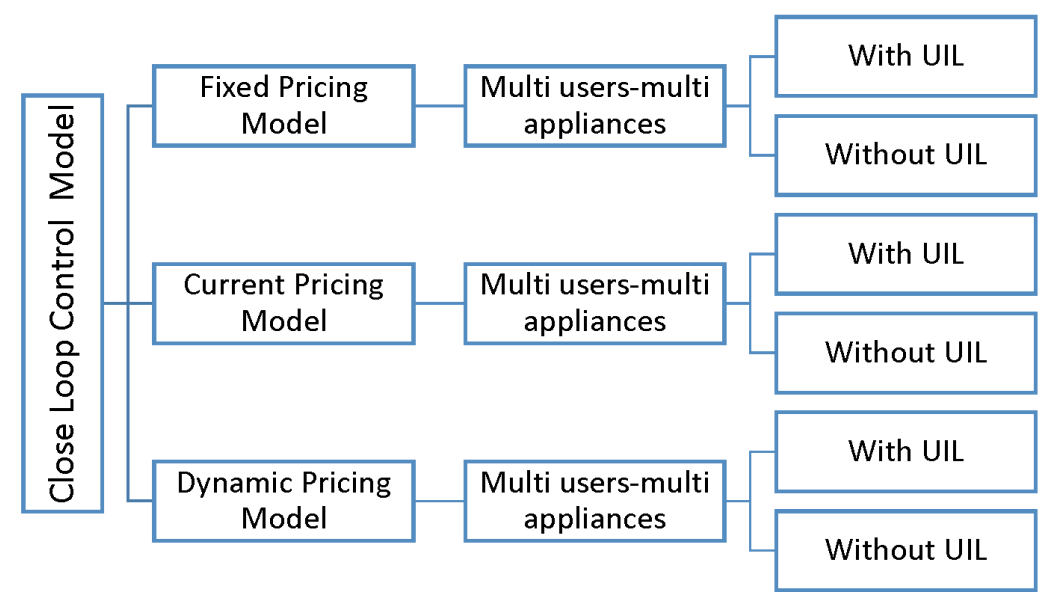

Figure 4.5: The Outline of Closed-Loop Control Model

of time, see Figure 4.4.

For a closed-loop control (CLC) model, we have examined the UIL method under three types of pricing models (fixed, current, and dynamic) in terms of multi usermulti appliance model, see Figure 4.5.

\subsection{Fixed Pricing Model}

Since the base price is stable during the whole year in the fixed pricing model, end user cannot earn any benefits from it. Therefore, it doesn't matter whether end users are a part of the system or not, see Figure 4.6. 


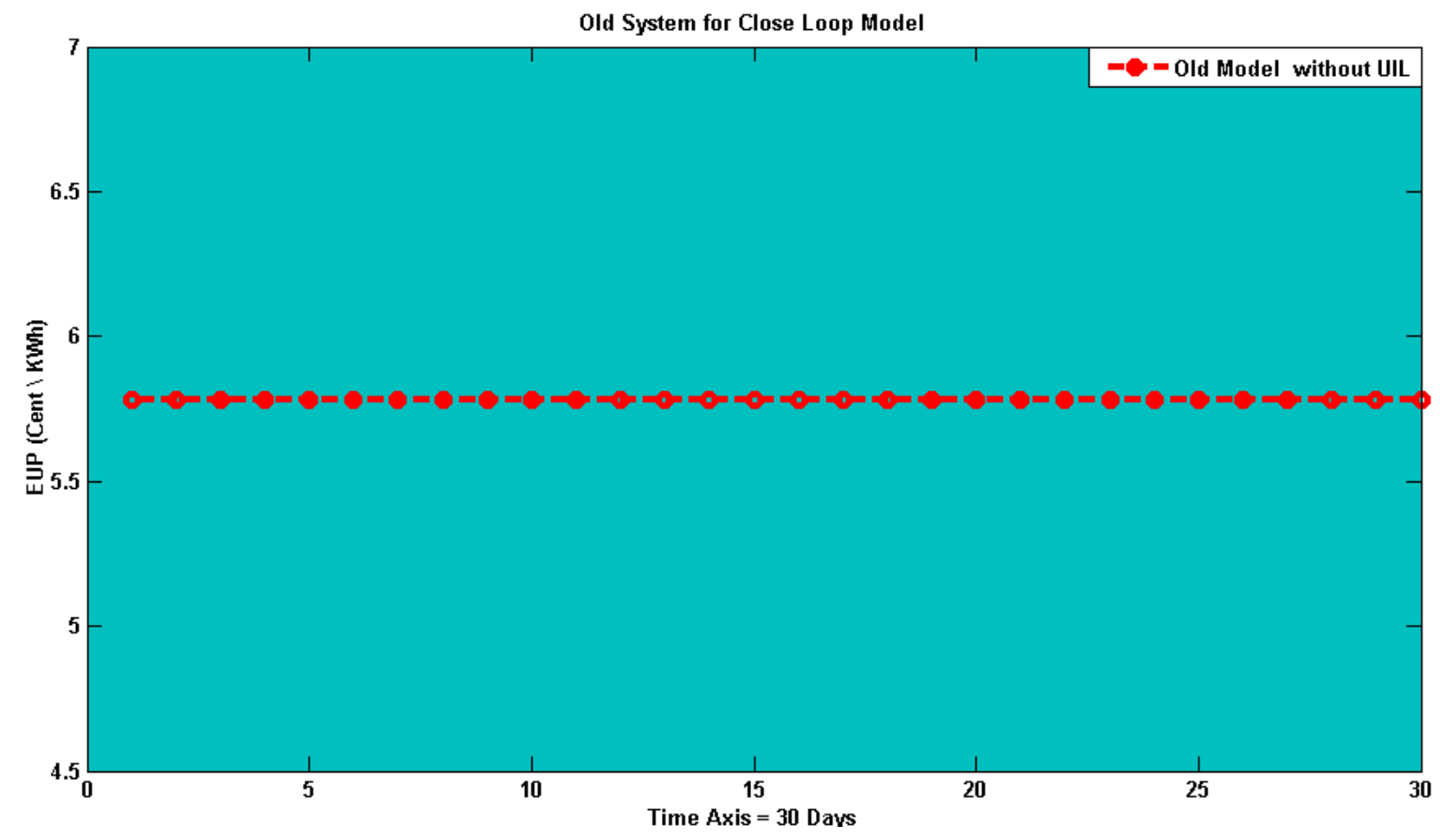

Figure 4.6: Fixed Pricing System for Closed-Loop Model

\subsection{Current Model}

In the current model, if users are a part of the system they can obtain some advantages. First, they can manage their usage depending on when the electricity price is low. As seen in Figure 4.7, user's payment doesn't exceed $\tau_{2}$ value. Second, by shaping their demand in time domain, they prevent high load congestion. Since they shape their demands, they help to shape supply. As a result, the supply and demand get closer to each other. Third, user awareness toward green energy increases. 


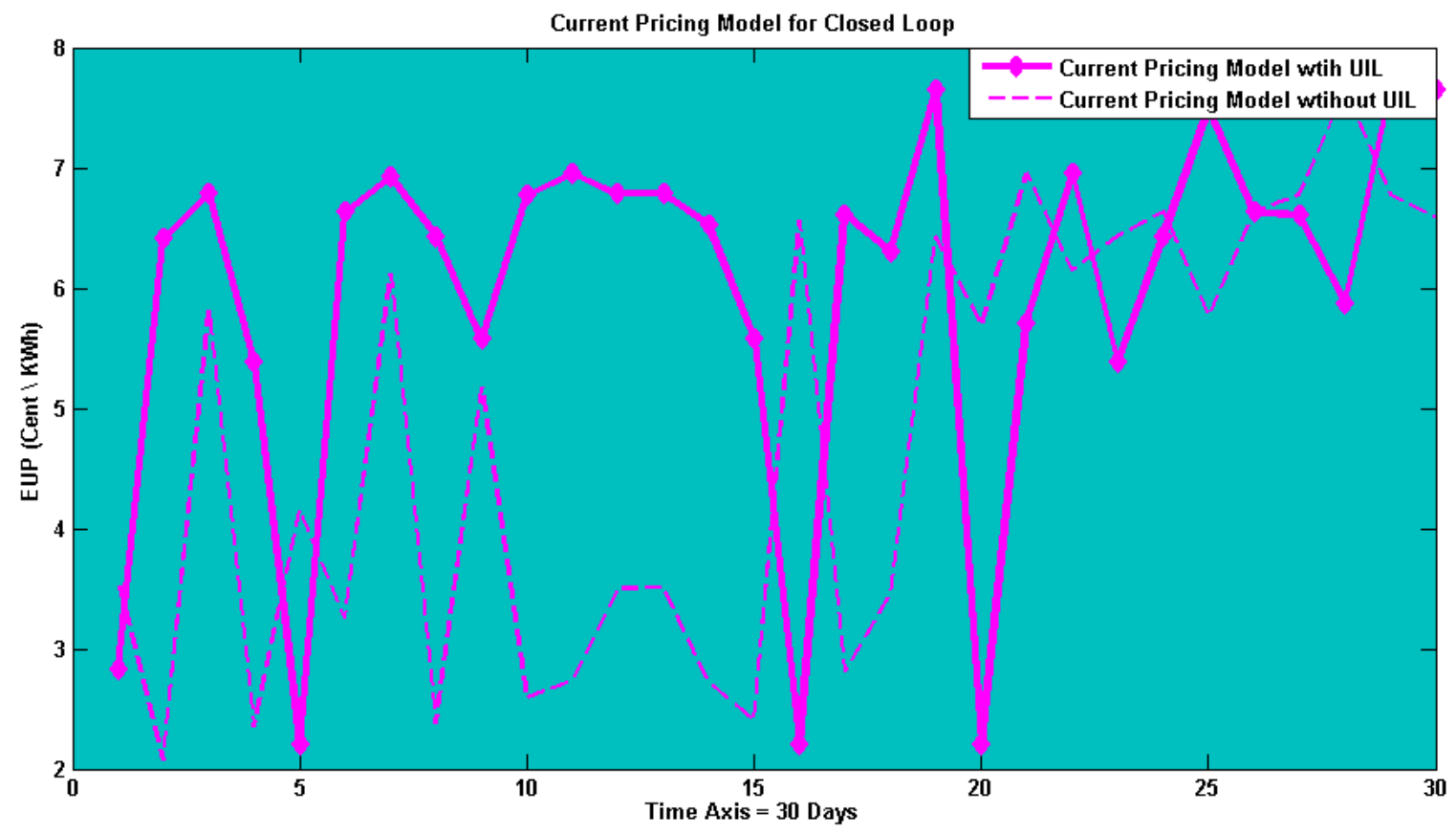

Figure 4.7: Current Pricing System for Closed-Loop Model

\subsection{Dynamic Pricing Model}

Threshold values are crucial in the dynamic pricing model, because the systems without these values turn into regular systems that users cannot get any benefit. Currently, HydroOne does not provide such a system for its users.

In the UIL model, end users are assumed to make a quick decision based on the relatively lower prices. In the closed-loop control model with the UIL model, the EUP price goes down after $\tau_{1}$ and getting closer to $\alpha$ as seen in Figure 4.8.

\subsection{Summary of This Chapter}

The term of the closed-loop control always implies the use of a feedback control sensor in order to determine and minimize the amount errors within the system. 


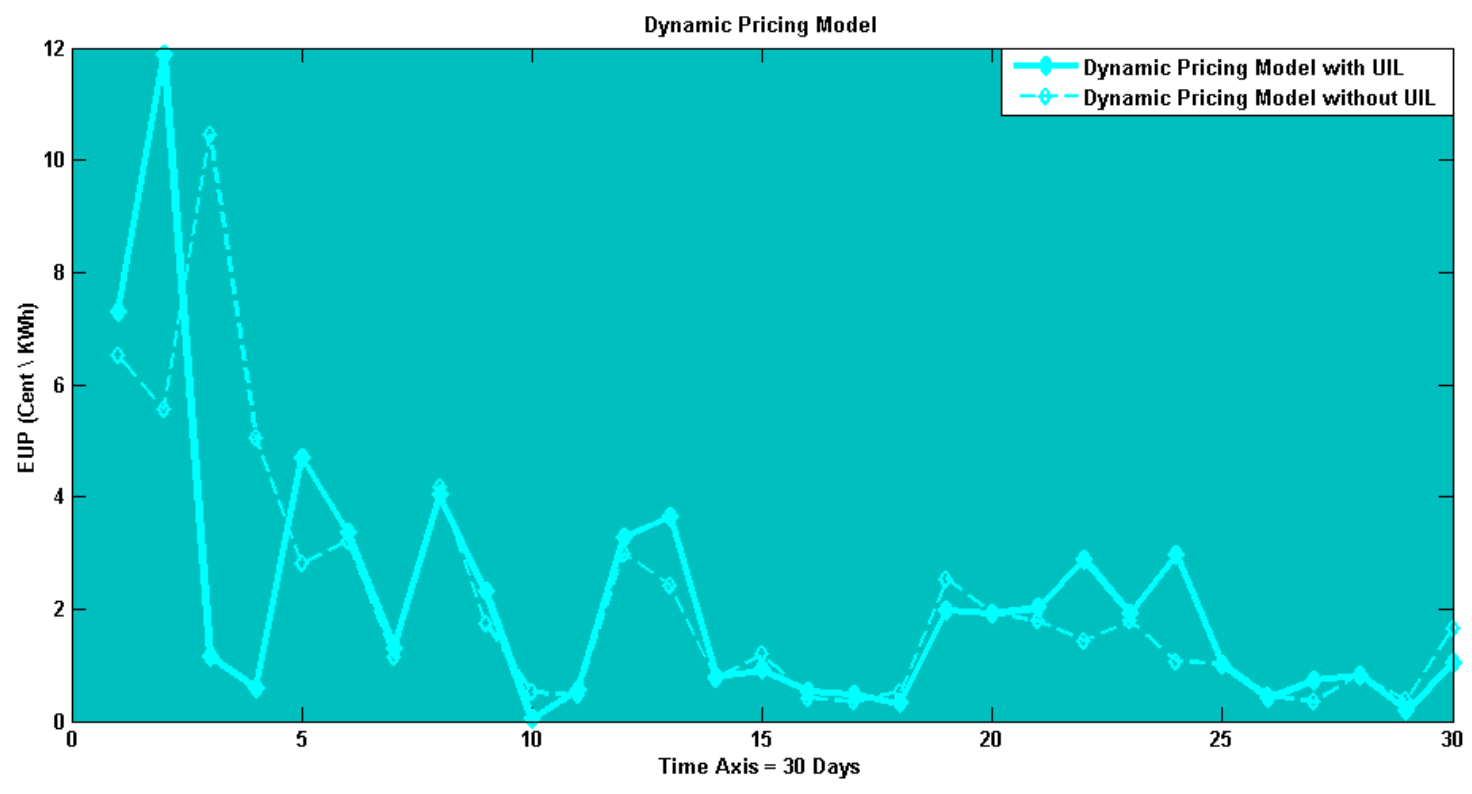

Figure 4.8: Dynamic Pricing System for Closed-Loop Model

In the closed-loop control model, we have examined the effectiveness of UIL method on 3 different pricing model regarding multi-user multi-appliance. Chapter 4 indicates that closed-loop control model is suitable for a large number of users and appliances. On the other hand, if the number of users and appliances is fewer, the number of low price periods increases. 


\section{Chapter 5}

\section{Conclusion and Future Works}

In statistics, the coefficient of determination, denoted $R^{2}$ and pronounced $\mathrm{R}$ squared, is a number that indicates how well data fit a statistical model, sometimes simply a line or curve. However, it is not necessary that the high value of $R^{2}$ is good.

As seen from Figure 5.1 and Figure 5.2, there is a parallel relation between supply, demand, and base price curves. This means that when the demand goes up, the supply goes up. Then the price is adjusted based on this relation. However, this price is the average of one month. When it comes to a one day relation, it is totally different. We understand from demand and supply formula that the convergence between them is more than $90 \%$, while it is approximately $50 \%$ for the base price see Figure 5.3 . Therefore, it will be a good idea to compare the base price to supply and demand to make a perfect realization.

\section{$5.1 \quad$ Open-Loop Results}

Here is the relation between all three kinds of pricing models with and without the UIL method. In Figure 5.4, if we use the UIL method instead of the regular one, we 


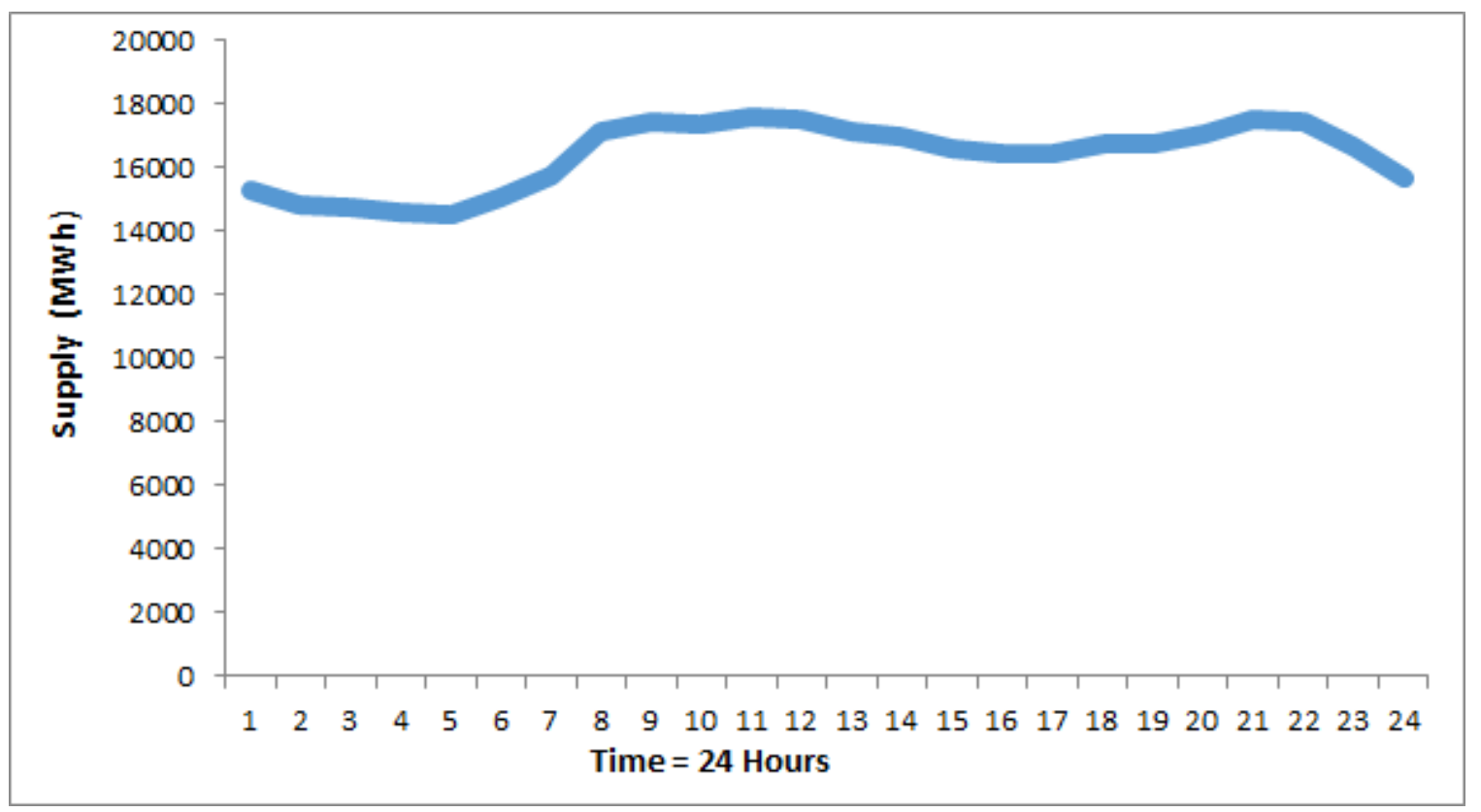

Figure 5.1: Supply Curve

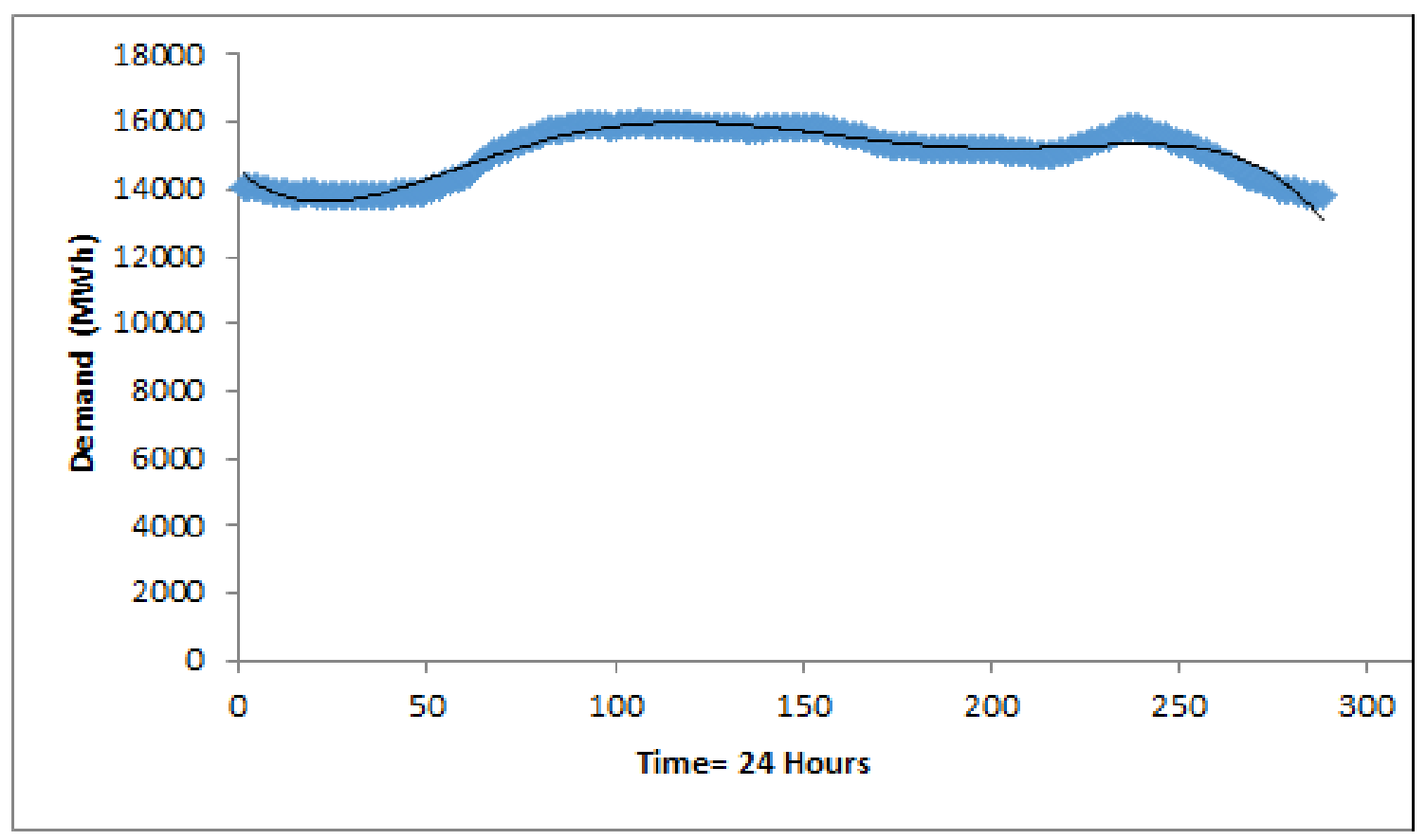

Figure 5.2: Demand Curve 


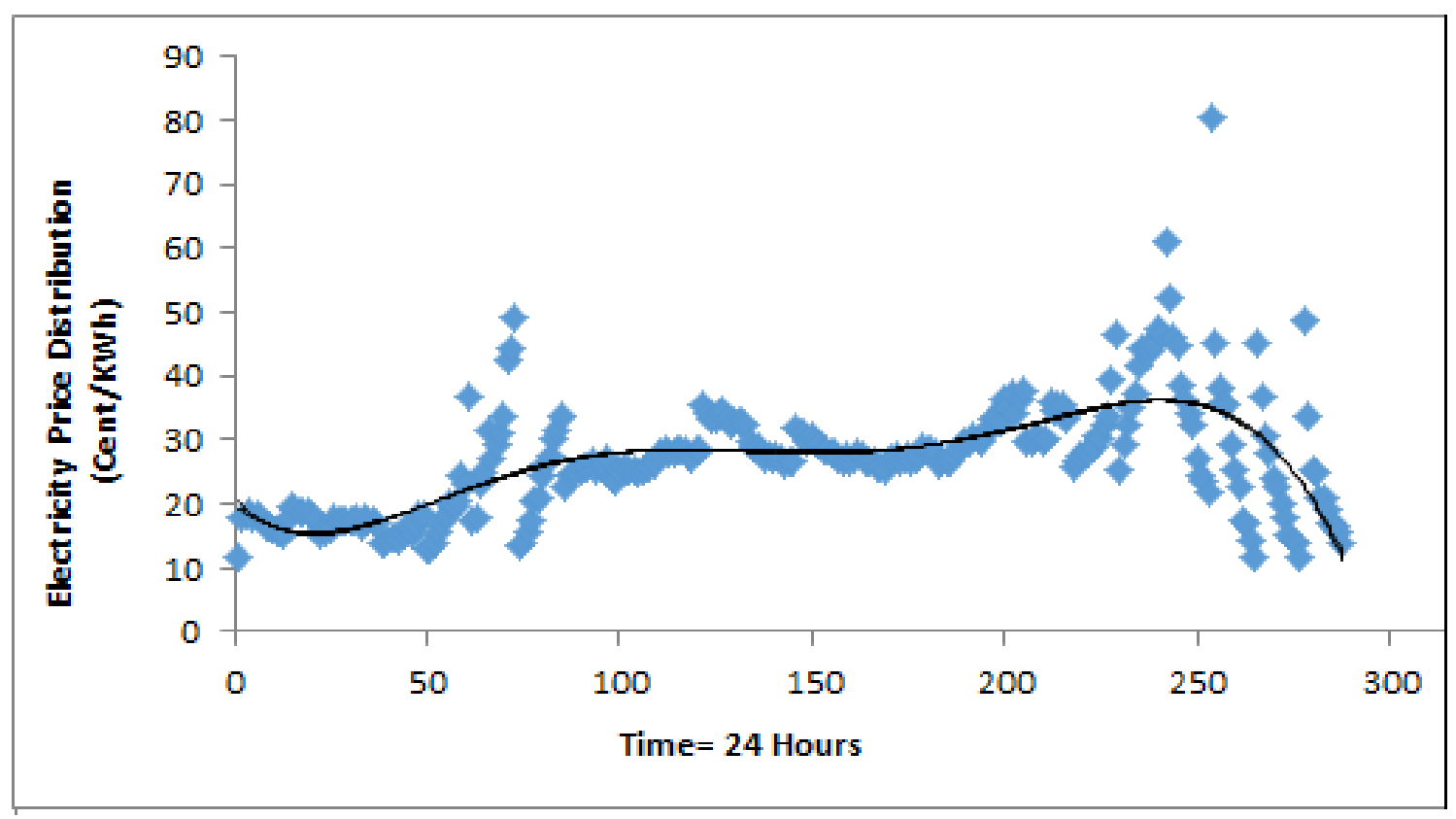

Figure 5.3: Electricity Price Distribution

basically save $23 \%$ in the current pricing model. A dynamic pricing model has more than an $8 \%$ improvement when we use UIL in the total in Figure 5.5.

When the number of user and appliances increases we still save but not as high as the one user-one appliance method.

\subsection{Closed-Loop Results}

In Chapter 4, we only simulated for multi-user multi-appliance. For the current pricing model, the total saving is around 5\%. Since we have used PID in closed-loop controller, the error between EUP and feedback price is going down and reach to zero. Total savings, for dynamic pricing, is about 7.5\%. As can be seen from Figure 5.6 , in a long period, in the dynamic pricing model, the UIL has higher advantages than without using the UIL. 


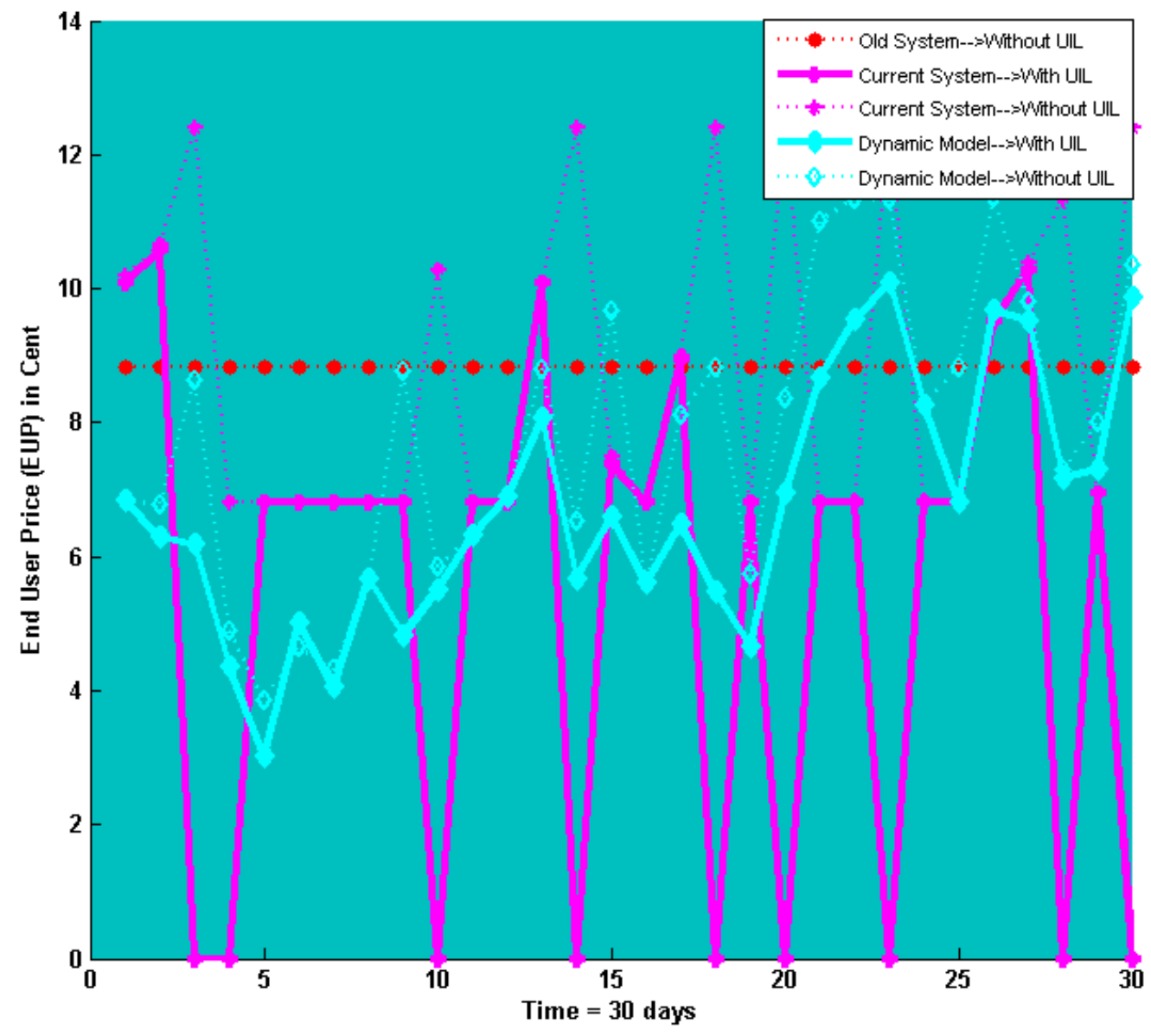

Figure 5.4: One-User One-Appliance 


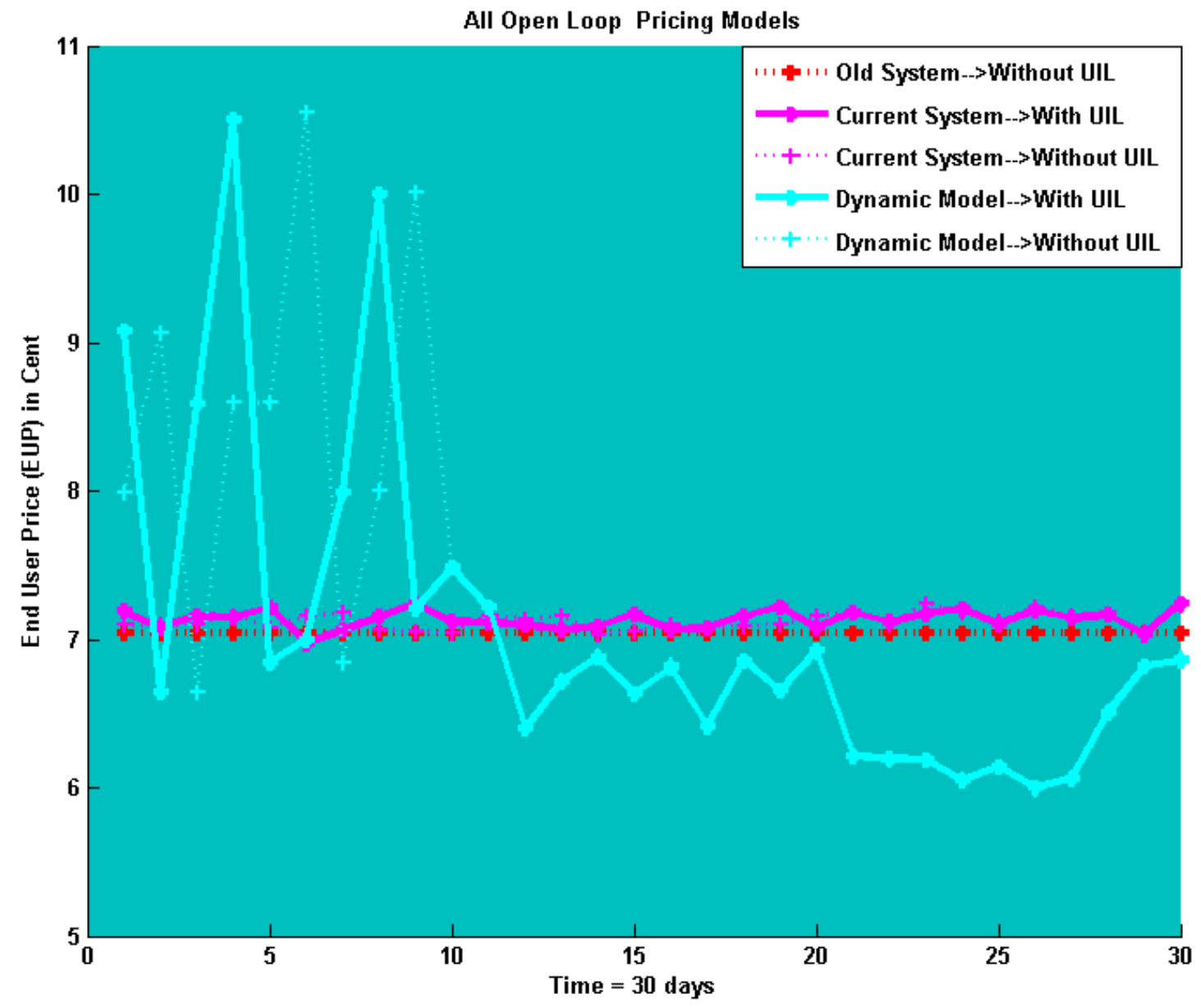

Figure 5.5: Multi-User Multi-Appliance Model for Open-Loop Control Model 


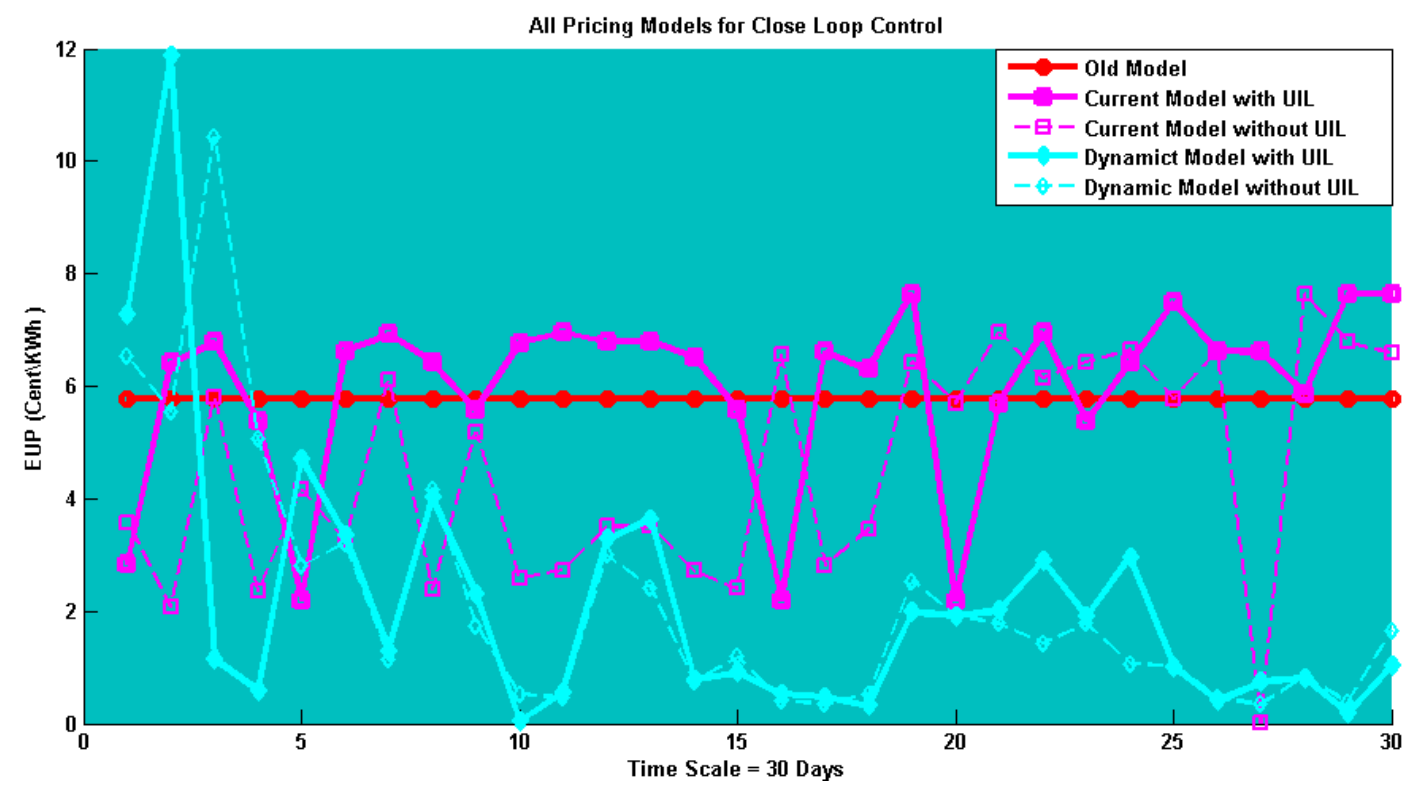

Figure 5.6: Multi-User Multi-Appliance Model for Closed-Loop Control Model

\begin{tabular}{|c|c|c|c|c|}
\hline Region & Threshold-Cent & EUP (Old Model)-Cent & EUP (Current Model)-Cent & EUP (Our Model Dynamic)-Cent \\
\hline green & 2.5 & 9.97 & 7.7 & 7.85 \\
\hline Yellow & 4.1 & 9.97 & 11.4 & 11.594 \\
\hline Red (Not Used) & $>4.1$ & 9.97 & 14 & $>11.594$ \\
\hline
\end{tabular}

Table 5.1: Model Pricing Comparisons

This table indicate the limit of threshold values and bound of colors, see Table 5.1.

\subsection{Conclusion}

In this study, we have compared both open and closed models for different scenarios. In each scenario, we compared three pricing model with UIL and without UIL. 


\begin{tabular}{|c|c|c|c|c|c|c|c|}
\hline \multirow{2}{*}{ Systems } & \multirow{2}{*}{$\begin{array}{l}\text { Pricing } \\
\text { Models / \# of } \\
\text { Users }\end{array}$} & \multicolumn{3}{|c|}{ One-User One Appliance Model } & \multicolumn{3}{|c|}{ Multi-User Multi-Appliance Model } \\
\hline & & Without UIL & With UIL & Improvement & Without UIL & With UIL & Improvement \\
\hline \multirow{3}{*}{$\begin{array}{l}\text { Open-Loop } \\
\text { Model }\end{array}$} & Fixed & 9.97 & 9.97 & 0 & 7 & 7 & 0 \\
\hline & Current & 10 & 8 & $20 \%$ & 7.2 & 7 & $2.7 \%$ \\
\hline & Dynamic & 9.5 & 7.5 & $21 \%$ & 7.3 & 6.72 & $7.9 \%$ \\
\hline \multirow{3}{*}{$\begin{array}{l}\text { Closed-Loop } \\
\text { Model }\end{array}$} & Fixed & - & - & - & 5.75 & 5.75 & 0 \\
\hline & Current & - & - & - & 5.62 & 5.42 & $3.5 \%$ \\
\hline & Dynamic & - & - & - & 2.54 & 2.26 & $11 \%$ \\
\hline
\end{tabular}

Figure 5.7: Comparison of Simulated Models with Different Number of Users w/o UIL Method

Additionally, we did these simulations for both one-user one-appliance and multiuser multi-appliance. From this thesis, we understood that UIL method works well especially for a low number of user and appliances. When the number of user and appliances increases, the total saving falls down slightly. In total, when users participated to the system and started being an active energy saver by adjusting their demand based on time and low price, they saved in energy and money. As a result, suppliers do not need to generate extra energy. They start to generate according to the demand from the consumers. There will be a tight interaction between consumer, supplier, smart appliance and cellphones. We use two way communications both in wire line and wireless.

Wireless communication is very important to set up such system, to communicate fast. Users have to transmit their demand to the supplier in a very short period.. Likewise, ZegBee communication is important to set interaction between devices and appliances. 


\subsubsection{Improvements Over the Current Pricing Model and Fu- ture Works}

- $7 \%-11 \%$ money efficiency,

- $12 \%$ money efficiency from supplier side,

- $7.5 \%-12.5 \%$ energy efficiency,

- Environmentally friendly, less CO emission,

- Increase end user awareness,

- Attention to renewable energy usage,

- More smart and safe system,

- Real time monitoring and reaction,

- Individual energy management,

- Prevention waste energy,

- Direct communication to end user customers,

- Maintain global competition.

We did limited research on smart grid communication; we did some assumptions in some parameters. For a more realistic model:

- Human behaviours have to be understood for precise assumptions,

- Sensors and some statistical measurements can be used to realize the resultsm,

- Since the system needs hundreds of data, the "Big Data" concept can be introduced to the systems, 
- Our system is good for a limited number of users and appliances; therefore big data can be used to extend the model for millions of users, smart phones, laptops and smart appliances,

- We have used real data for our system. However, in reality we don't have such data in advance. Therefore, some filter techniques could be used for base price estimations. Kalman filters and the moving average (MA) method can be integrated to this system for making electricity price assumptions. 


\section{List of References}

[1] Y. Martel, "The internet of things." http://www.opinno.com/en/content/ internet-things-0? language=es. [Accessed: 12- Feb- 2013].

[2] HydroOne, "Automated meter reading." http://www.hydroone.com/ myhome/myaccount/mymeter/pages/automatedmeterreading. aspx [Accessed: 03- May- 2015].

[3] N. on the Lake Hydro, "Niagara on the lake." http://www.notlhydro.com/ smartmeters/pricing [Accessed: 03- May- 2015].

[4] O. E. Board, "Electricity prices." http://www.ontarioenergyboard.ca/OEB/ Consumers/Electricity/Electricity+Prices [Accessed: 03- May- 2015].

[5] R. Aulana, "Plc (power line communication)." http://rustammaulana. blogspot.ca/2012/10/plc-power-line-communication.html. [Accessed: 05May- 2015].

[6] R. Schoenen and H. Yanikomeroglu, "Dynamic demand control with differentiated qos in user-in-the-loop controlled cellular networks," in IEEE 77th Vehicular Technology Conference (VTC Spring), pp. 1-6, 2013.

[7] Kanda, "Zigbee wireless interfaces and products." http://www.kanda.com/ zigbee-wireless.html [Accessed: 06- May- 2015].

[8] Electronicdesign, "Use zigbee for cost-effective wpan sensing and control solutions - proactive, logical, and well-organized design evaluation with a solid reference design helps simplify wireless personal-area networks (wpan) design and development - communications content from electronic design." http://electronicdesign.com/communications/ use-zigbee-cost-effective-wpan-sensing-and-control-solutions [Accessed: 06- May- 2015]. 
[9] Z. Economics, "Supply and demand." https://zahablog.wordpress.com/ concepts/basics-and-markets/supply-and-demand-2. [Accessed: 03- May2015].

[10] O. Hydro, "Ontario time-of-use electricity rates." http://www . ontario-hydro. com/index .php?page=current_rates[Accessed: 03- May- 2015].

[11] M. Mirahsan, Z. Wang, R. Schoenen, H. Yanikomeroglu, and M. St-Hilaire, "Unified and non-parameterized statistical modeling of temporal and spatial traffic heterogeneity in wireless cellular networks," in IEEE International Conference on Communications Workshops (ICC) 2014 Workshop on 5G Technologies, pp. 5560, 10-14 June 2014.

[12] R. Schoenen, G. Bulu, A. Mirtaheri, T. Beitelmal, and H. Yanikomeroglu, "First survey results of quantified user behavior in user-in-the-loop scenarios for sustainable wireless networks," in IEEE Vehicular Technology Conference (VTC Fall), pp. 1-5, 2012.

[13] J. Holler, V. Tsiatsis, C. Mulligan, S. Avesand, S. Karnouskos, and D. Boyle, From Machine-to-machine to the Internet of Things: Introduction to a New Age of Intelligence. Academic Press, 2014.

[14] E. Evolving, "Analytics, Machine Learning, and the Internet of Things,"

[15] A. B. Intelligence, "More than 30 billion devices will wirelessly connect to the internet of everything in 2020," ABI research news, vol. 9, 2013.

[16] N. Kushalnagar, G. Montenegro, C. Schumacher, et al., "Ipv6 over low-power wireless personal area networks (6lowpans): overview, assumptions, problem statement, and goals," RFC4919, vol. 10, August 2007.

[17] R. Schoenen, G. Bulu, A. Mirtaheri, and H. Yanikomeroglu, "Green communications by demand shaping and user-in-the-loop tariff-based control," in IEEE Online Conference on Green Communications (GreenCom), pp. 64-69, 2011.

[18] I. Richardson, M. Thomson, D. Infield, and C. Clifford, "Domestic electricity use: A high-resolution energy demand model," Energy and Buildings, vol. 42, no. 10, pp. 1878-1887, 2010.

[19] F. Rahimi and A. Ipakchi, "Demand response as a market resource under the smart grid paradigm," IEEE Transactions on Smart Grid, vol. 1, no. 1, pp. 82$88,2010$. 
[20] W. Li and X. Zhang, "Simulation of the smart grid communications: Challenges, techniques, and future trends," Computers 86 Electrical Engineering, vol. 40, no. 1, pp. 270-288, 2014.

[21] M. Aydinalp, V. I. Ugursal, and A. S. Fung, "Modeling of the appliance, lighting, and space-cooling energy consumptions in the residential sector using neural networks," Applied Energy, vol. 71, no. 2, pp. 87-110, 2002.

[22] H. Mouftah and M. Erol-Kantarci, "Smart grid communications: Opportu nities and challenges," Handbook on Green Communication and Systems, edited by MS Obaidat, A. Anpalagan and I. Woungang, Wiley, 2012.

[23] A. Ghassemi, S. Bavarian, and L. Lampe, "Cognitive radio for smart grid communications," in IEEE First International Conference on Smart Grid Communications (SmartGridComm), pp. 297-302, 2010.

[24] D. Dzung, I. Berganza, and A. Sendin, "Evolution of powerline communications for smart distribution: from ripple control to OFDM," in IEEE International Symposium on Power Line Communications and Its Applications (ISPLC), pp. 474-478, 2011.

[25] Z. Wang, R. Schoenen, H. Yanikomeroglu, and M. St-Hilaire, "Load balancing in cellular networks with user-in-the-loop: A spatial traffic shaping approach," in IEEE International Conference on Communications (ICC). London, UK, 2015.

[26] R. Schoenen and H. Yanikomeroglu, "User-in-the-loop: spatial and temporal demand shaping for sustainable wireless networks," IEEE Communications Magazine, vol. 52, no. 2, pp. 196-203, 2014.

[27] R. Schoenen and H. Yanikomeroglu, "Erlang analysis of cellular networks using stochastic petri nets and user-in-the-loop extension for demand control," in IEEE Globecom Workshops (GC Wkshps), pp. 298-303, 2013.

[28] R. Schoenen, G. Bulu, A. Mirtaheri, T. Beitelmal, and H. Yanikomeroglu, "Quantified user behavior in user-in-the-loop spatially and demand controlled cellular systems," in European Wireless, 2012. EW. 18th European Wireless Conference, pp. 1-8, 2012.

[29] T. Beitelmal, R. Schoenen, and H. Yanikomeroglu, "On the impact of correlated shadowing on the performance of user-in-the-loop for mobility," in IEEE International Conference on Communications (ICC), pp. 7040-7044, 2012. 
[30] J. H. Yoon, R. Baldick, and A. Novoselac, "Dynamic demand response controller based on real-time retail price for residential buildings," IEEE Transactions on Smart Grid, vol. 5, no. 1, pp. 121-129, 2014.

[31] A. J. Conejo, J. M. Morales, and L. Baringo, "Real-time demand response model," IEEE Transactions on Smart Grid, vol. 1, no. 3, pp. 236-242, 2010.

[32] W. H. Chin, Z. Fan, and R. Haines, "Emerging technologies and research challenges for 5G wireless networks," IEEE Wireless Communications, vol. 21, no. 2, pp. 106-112, 2014. 


\section{Appendix A}

\section{Equations}

\section{Supply Formula:}

$y=-0.0031 x^{6}+0.1743 x^{5}-2.9319 x^{4}+3.7674 x^{3}+277.09 x^{2}-1589.6 x+16743(K W h)$
$R^{2}=0.9489$

Demand Formula:

$$
\begin{aligned}
& y=(-1 E-07) x^{5}+0.0001 x^{4}-0.0268 x^{3}+2.8439 x^{2}-91.905 x+14541(K W h) \\
& R^{2}=0.9373
\end{aligned}
$$

\section{Price Distribution:}

$y=(-1 E-09) x^{5}+(7 E-07) x^{4}-0.0002 x^{3}+0.0159 x^{2}-0.4658 x+19.738$ (Cent per KWh)

$R^{2}=0.4793$ 